\title{
The roles of RNA helicases and other ribosome biogenesis factors during small subunit maturation
}

\author{
Dissertation \\ for the award of the degree \\ "Doctor rerum naturalium" \\ of the Georg-August-Universität Göttingen \\ within the doctoral program Molecular Biology of Cells \\ of the Georg-August University School of Sciences (GAUSS) \\ submitted by \\ Jimena Davila Gallesio \\ from Paso de los Libres, Argentina
}

Göttingen, 2019 


\title{
Thesis Committee
}

Prof. Dr. Markus Bohnsack

Prof. Dr. Jörg Enderlein

Prof. Dr. Gerhard Braus
Department of Molecular Biology

University Medical Centre Göttingen

III. Physical Institute

Georg-August University Göttingen

Institute for Microbiology and Genetics

Georg-August University Göttingen

\section{Members of the Examination Board}

\author{
Reviewer: Prof. Dr. Markus Bohnsack \\ Department of Molecular Biology \\ University Medical Centre Göttingen \\ $2^{\text {nd }}$ Reviewer: Prof. Dr. Jörg Enderlein \\ III. Physical Institute \\ Georg-August University Göttingen
}

\section{Further members of the Examination Board}

Prof. Dr. Blanche Schwappach-Pignataro

Prof. Dr. Michael Thumm

Prof. Dr. Michael Meinecke

Prof. Dr. Ralf Ficner
Department of Molecular Biology

University Medical Centre Göttingen

Department of Cellular Biochemistry

University Medical Centre Göttingen

Department of Cellular Biochemistry

University Medical Centre Göttingen

Department of Structural Molecular Biology

Georg-August University Göttingen 
Table of Contents

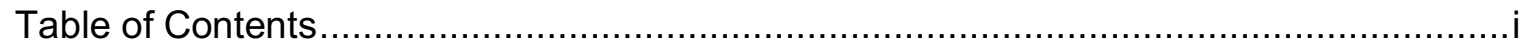

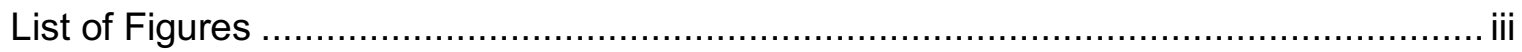

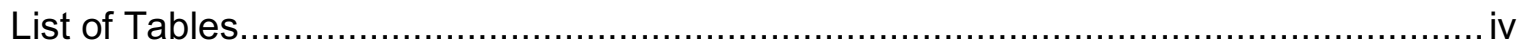

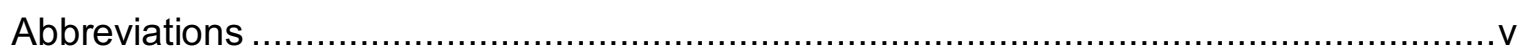

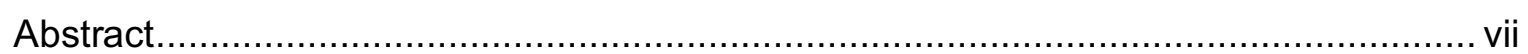

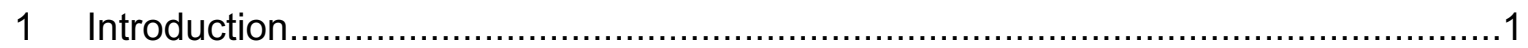

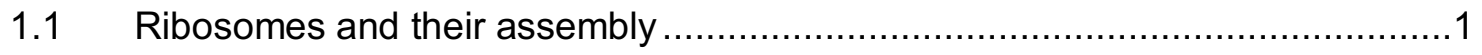

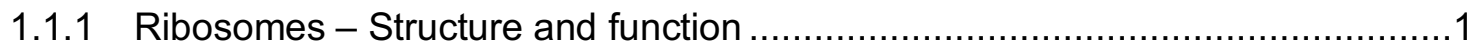

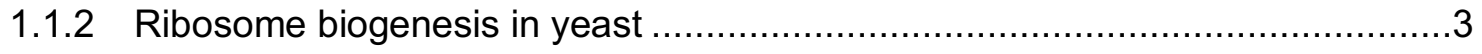

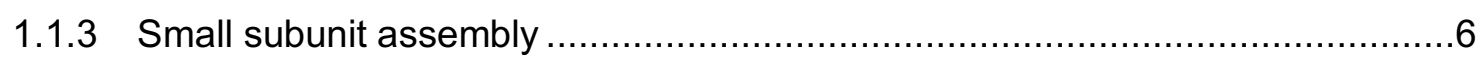

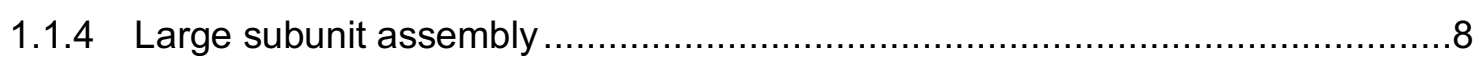

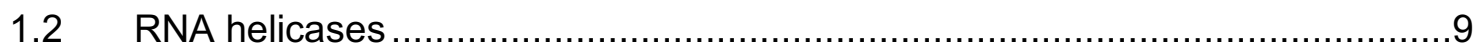

1.2.1 Helicase structure and classification in families.............................................

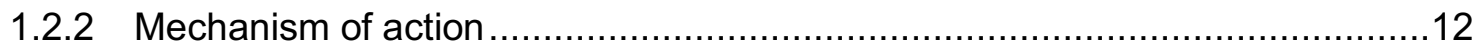

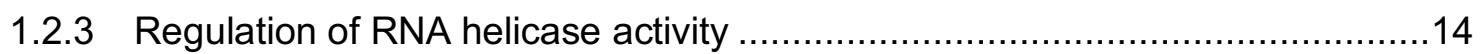

1.2.3.1 RNA helicase regulation by cofactor proteins ..................................14

1.2.3.2 Non-cofactor mediated mechanisms of RNA helicase regulation ............16

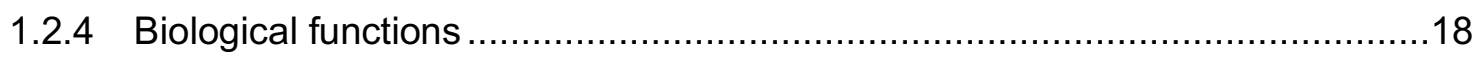

1.2.5 RNA helicases and their relation to diseases ...........................................20

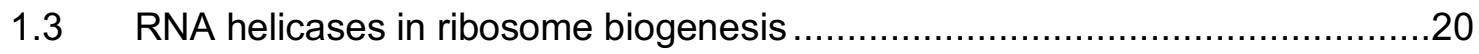

1.3.1 The roles of RNA helicases in regulating snoRNA dynamics on pre-ribosomes.

1.3.2 The roles of RNA helicases as structural remodellers on pre-ribosomes........22

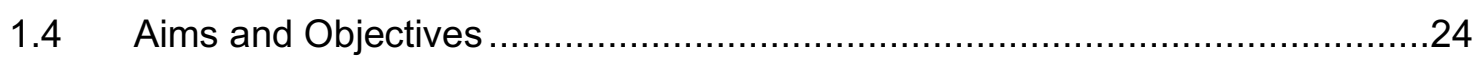

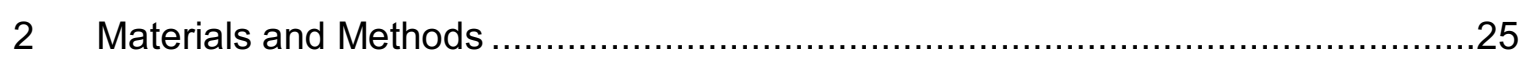

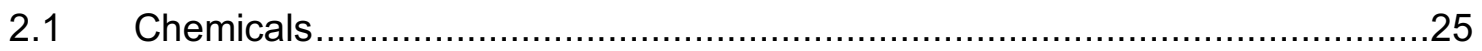

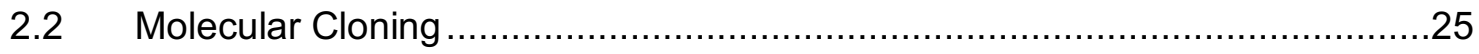

2.2.1 DNA amplification by PCR and detection of product .................................25

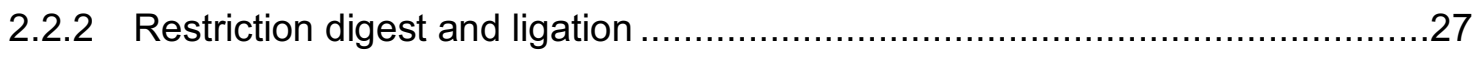

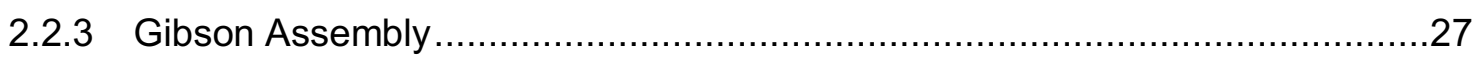

2.2.4 E. coli transformation and extraction of plasmid DNA ..................................28

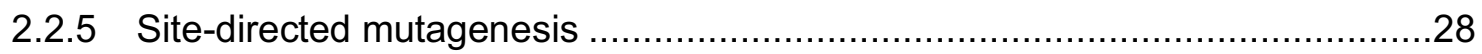

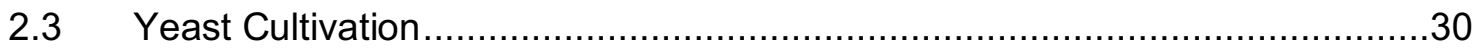

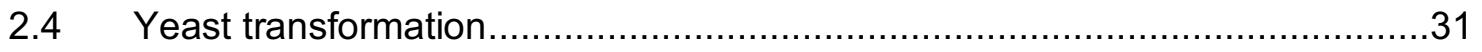

2.5 Depletion of endogenous proteins from yeast using the auxin-inducible degron system 


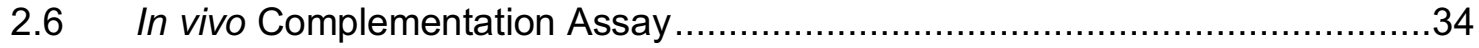

2.7 Enrichment of endogenous complexes on IgG sepharose ...........................34

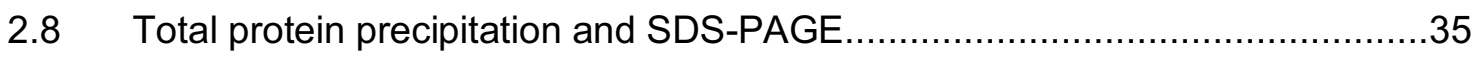

2.9 Western blotting and Coomassie staining ................................................

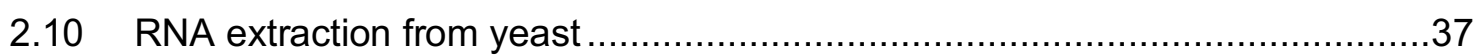

2.11 Denaturing agarose gel electrophoresis and northern blotting for pre-rRNAs..38

2.12 In culturo UV-crosslinking and analysis of cDNA (CRAC) .............................39

2.13 Recombinant protein expression in E. coli ...............................................42

2.14 Recombinant Protein Purification ..................................................................... 43

2.15 In vitro NADH-coupled ATPase assay ...................................................44

2.16 Anisotropy Measurements ............................................................... 44

2.17 Binding assay of recombinantly expressed proteins .....................................45

2.17.1 Binding assay using yeast lysate and purified recombinant proteins ..........45

2.17.2 Binding assay using purified recombinant proteins..................................46

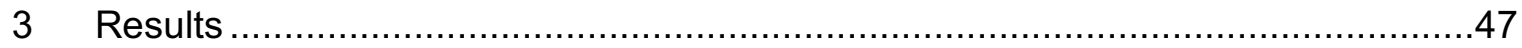

3.1 Depletion of Fal1 leads to pre-rRNA processing defects at cleavage sites $A_{0}, A_{1}$

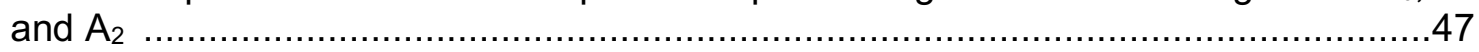

3.2 The ATPase activity of Fal1 is required for pre-rRNA processing ...................51

3.3 The MIF4G domain-containing protein Sgd1 interacts with Fal1 in vivo and depletion of Sgd1 leads to similar pre-rRNA processing defects as Fal1 depletion.....54

3.4 The MIF4G domain of Sgd1 binds to Fal1 and can stimulate the ATPase

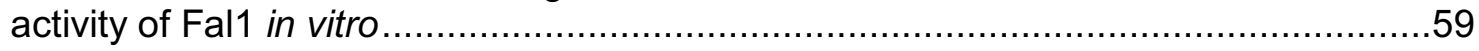

3.5 Sgd1 crosslinks to helix 12 of the $18 \mathrm{~S}$ rRNA sequence................................62

3.6 The C-terminal region of Sgd1 is responsible for RNA binding.....................67

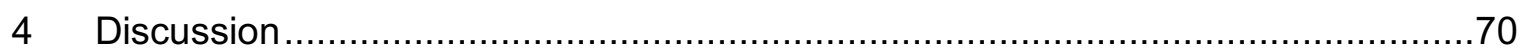

4.1 Identification of in vivo binding sites of ribosome assembly factors on pre-

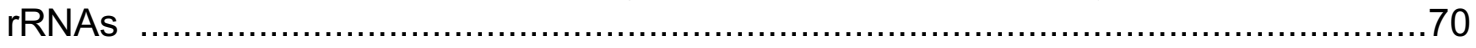

4.2 Molecular function of RNA helicases in ribosome biogenesis........................73

4.3 Alternative pre-rRNA processing pathways .......................................... 77

4.4 Recruitment and regulation of RNA helicases ....................................... 79

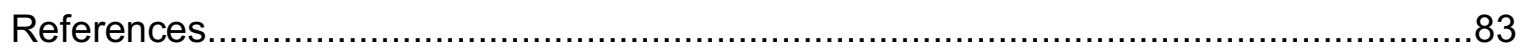

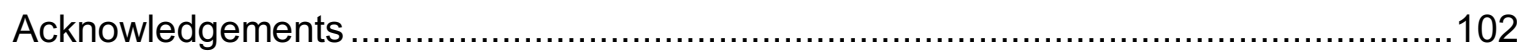

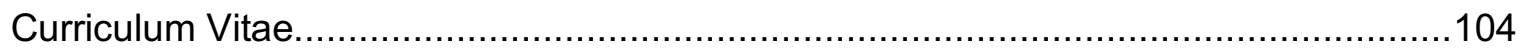




\section{List of Figures}

Figure 1.1 Secondary and tertiary structure of the S. cerevisiae rRNAs..........................

Figure 1.2 Eukaryotic ribosome assembly pathway....................................................

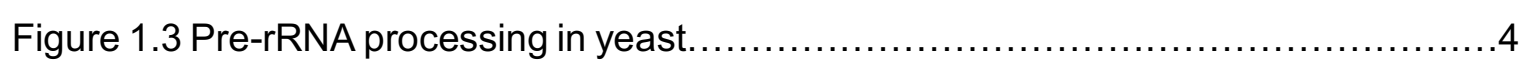

Figure 1.4 Organisation and structure of the helicase core of RNA helicases...............11

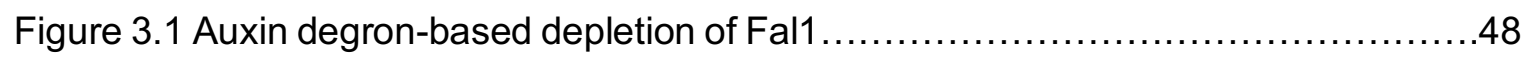

Figure 3.2 Depletion of Fal1 leads to SSU pre-rRNA processing defects..................49

Figure 3.3 Depletion of Fal1 leads to accumulation of 23S, 22S and $21 \mathrm{~S}$ aberrant prerRNAs.

Figure 3.4 Fal1 is an RNA-dependent ATPase, and mutation of the DEAD sequence within

Motif II impairs ATPase activity in vitro....................................52

Figure 3.5 The ATPase activity of Fal1 is required for SSU pre-rRNA processing ...........53

Figure 3.6 Auxin degron-based depletion of Sgd1 ................................... 55

Figure 3.7 Depletion of Sgd1 leads to similar defects in pre-rRNA processing to Fal1

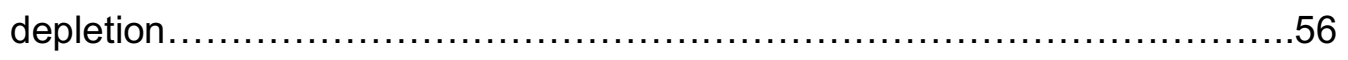

Figure 3.8 Recombinant expression and purification of Sgd1 from E. coli..................57

Figure $3.9 \mathrm{Fal} 1$ is retrieved from yeast cell extracts by Sgd1 ........................... 58

Figure 3.10 Recombinant expression and purification of full-length Sgd1 and Sgd1

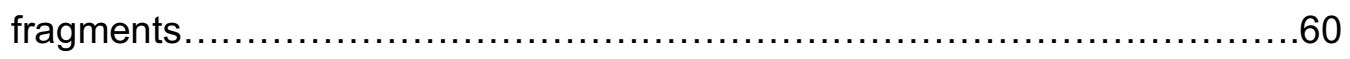

Figure $3.11 \mathrm{Sgd} 1$ binds to Fal1 through its MIF4G domain............................61

Figure 3.12 The MIF4G domain of Sgd1 can stimulate the ATPase activity of Fal1 in

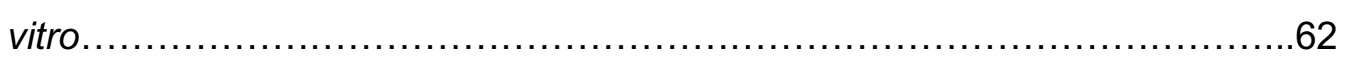

Figure 3.13 Sgd1 specifically crosslinks to RNA in vivo.............................63

Figure 3.14 Sgd1-crosslinked RNA fragments are derived from rRNA ...................64

Figure $3.15 \mathrm{Sgd} 1$ crosslinks within the $18 \mathrm{~S}$ rRNA sequence of $35 \mathrm{~S}$ rRNA.................65

Figure $3.16 \mathrm{Sgd} 1$ crosslinks to helix 12 of $18 \mathrm{~S}$ rRNA sequence........................66

Figure 3.17 The RNA binding site of Sgd1 is located within a binding pocket in the SSU processome structure.................................................

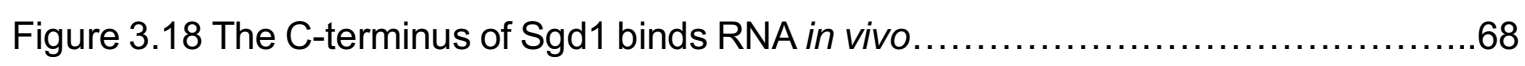

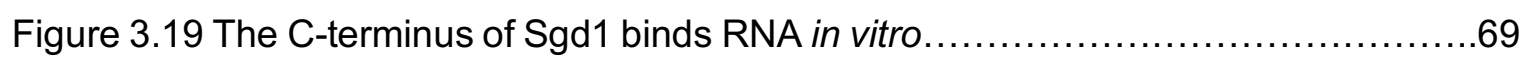




\section{List of Tables}

Table 2.1 Oligonucleotides used for molecular cloning and mutagenesis......................26

Table 2.2 Standard PCR reaction mix and cycle conditions......................................27

Table 2.3 Standard reaction mix and PCR conditions for site-directed mutagenesis. .....29

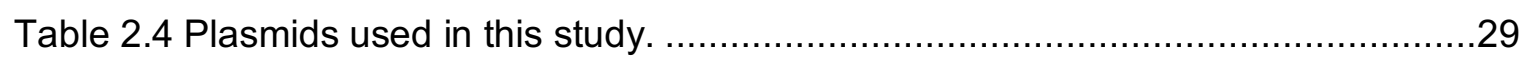

Table 2.5 Oligonucleotides used for amplification of tagging cassettes. ........................ 31

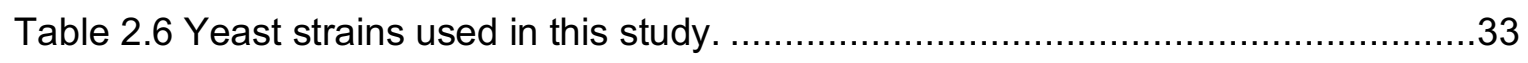

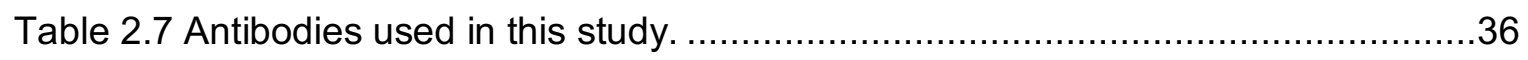

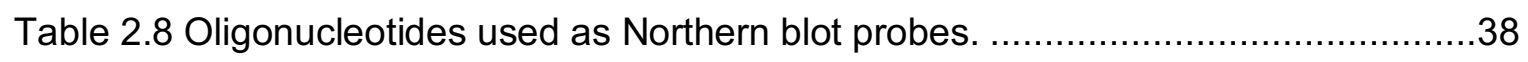

Table 2.9 Conditions for PCR amplification of cDNA . ........................................... 42 


\section{Abbreviations}

\begin{tabular}{|c|c|}
\hline Abbreviation & Meaning \\
\hline AF & Assembly factor \\
\hline AID & Auxin-inducible degradation \\
\hline ATP & Adenosine triphosphate \\
\hline cDNA & Complementary DNA \\
\hline CRAC & Crosslinking and analysis of cDNA \\
\hline Cryo-EM & Cryo-electron microscopy \\
\hline dNTP & Deoxynucleoside triphosphate \\
\hline dsRNA & Double-stranded RNA \\
\hline EJC & Exon-junction complex \\
\hline ETS & External transcribed spacer \\
\hline EV & Empty vector \\
\hline G-Patch domain & Glycine-rich domain \\
\hline $\mathrm{HA}$ & Hemagglutinin \\
\hline HTP & His $_{6}-$ TEV cleavage site-ProteinA \\
\hline HEAT & Huntingtin, eF3, 2A, TOR1 \\
\hline IAA & Indole-3-acetic acid, auxin \\
\hline ITS & Internal transcribed spacer \\
\hline $\mathrm{kDa}$ & Kilodalton \\
\hline IncRNA & Long non-coding RNA \\
\hline LSU & Large ribosomal subunit \\
\hline MIF4G & Middle domain of elF4G \\
\hline mRNA & Messenger RNA \\
\hline ncRNA & Non-coding RNA \\
\hline NTP & Nucleoside triphosphate \\
\hline ORF & Open reading frame \\
\hline PCR & Polymerase chain reaction \\
\hline PET & Peptide exit tunnel \\
\hline $\mathrm{Pi}$ & Inorganic phosphate \\
\hline $\mathrm{PI}$ & Protease Inhibitor \\
\hline Pol I / II / III & RNA Polymerase I / II / III \\
\hline PTC & Peptidyl transferase centre \\
\hline PTM & Post-translational modification \\
\hline rDNA & Ribosomal DNA \\
\hline
\end{tabular}




$\begin{array}{ll}\text { RNP } & \text { Ribonucleoprotein particle } \\ \text { RP } & \text { Ribosomal protein } \\ \text { rRNA } & \text { Ribosomal RNA } \\ \text { SDS-PAGE } & \text { Sodium dodecyl sulfate polyacrylamide gel electrophoresis } \\ \text { SF } & \text { Superfamily } \\ \text { SnoRNA } & \text { Small nucleolar RNA } \\ \text { snoRNP } & \text { Small nucleolar ribonucleoprotein particle } \\ \text { SnRNA } & \text { Small nuclear RNA } \\ \text { SSRNA } & \text { Single-stranded RNA } \\ \text { SSU } & \text { Small ribosomal subunit } \\ \text { TAP } & \text { Tandem affinity purification } \\ \text { TCA } & \text { Trichloroacetic acid } \\ \text { TEV } & \text { Tobacco etch virus } \\ \text { tRNA } & \text { Transfer RNA } \\ \text { UTR } & \text { Untranslated region }\end{array}$




\section{Abstract}

RNA helicases are a highly conserved family of proteins that act as RNA-dependent NTPases. These proteins contain a conserved helicase core consisting of two RecA-like domains that are responsible for unwinding or annealing RNA duplexes and remodelling RNP complexes in an NTP-dependent manner. As most RNA helicases perform their unwinding activity in a sequence independent manner, protein-protein interactions with cofactors can modulate their activity or provide substrate specificity. In line with their molecular functions, these proteins are central players in important cellular processes involving RNA, including pre-mRNA splicing, translation and ribosome biogenesis.

The production of mature eukaryotic ribosomes is a highly dynamic and energy-consuming process that involves four rRNAs, $\sim 80$ ribosomal proteins and more than 200 trans-acting factors. In the yeast S. cerevisiae, 21 RNA helicases are involved in the assembly steps of the small and large subunits (SSU and LSU respectively), where general roles have been attributed to RNA helicases in remodelling rRNAs and modulating the dynamics of small nucleolar (sno)RNPs on pre-ribosomes. In recent years, the identification of binding sites of different RNA helicases on the rRNA as well as structural analyses of preribosomal particles has facilitated a deeper understanding of how these proteins act in ribosome biogenesis. However, for other RNA helicases, the lack of information regarding their rRNA binding sites and their molecular targets has prevented further characterisation of their functions in ribosome biogenesis.

This study focused on the uncharacterised DEAD-box helicase Fal1 and the MIF4G domain-containing protein Sgd1, which are both required for SSU maturation. Analyses of pre-rRNA processing upon protein depletion demonstrated that Fal1 and Sgd1 are both required for early pre-rRNA cleavages at sites $A_{0}, A_{1}$ and $A_{2}$, and complementation experiments showed that the ATPase activity of the helicase is required for this function. Fal1 and Sgd1 were shown to associate in vivo, and in vitro analyses determined that the MIF4G domain of Sgd1 mediates the interaction with Fal1. Excitingly, the data suggest that the MIF4G domain of Sgd1 can stimulate the ATPase activity of Fal1 in vitro, suggesting a role of Sgd1 as an MIF4G domain-containing cofactor of Fal1. The UV crosslinking and analysis of cDNA (CRAC) approach allowed the identification of a Sgd1 binding site within the 18S rRNA sequence, which is in line with a suggested role in the early stages of pre-SSU assembly. Interestingly, expression of different Sgd1 truncations for in vivo crosslinking experiments highlighted the C-terminal region of Sgd1 as responsible for the association with RNA. Anisotropy experiments demonstrated that the C-terminal region of Sgd1 can bind RNA in vitro in a non-sequence specific manner, 
Abstract

suggesting that Sgd1 can simultaneously bind Fal1 through the MIF4G domain and the rRNA through the C-terminal region. Altogether, these findings expand our understanding of the role of Fal1 and Sgd1 in ribosome biogenesis, and suggest a common function of these proteins in the early stages of ribosome assembly, likely as an RNA helicasecofactor complex. 


\section{Introduction}

\subsection{Ribosomes and their assembly}

\subsubsection{Ribosomes - Structure and function}

In all living cells, the information contained in messenger (m)RNAs is translated into proteins by ribosomes. Ribosomes are highly complex ribonucleoprotein complexes (RNPs), organised as two asymmetric subunits: a small subunit (SSU) and a large subunit (LSU). Each subunit carries out different functions in translation; the decoding centre of the SSU is responsible for base-pairing of mRNA codon triplets with the anticodons of the corresponding aminoacylated transfer RNAs (tRNAs), whereas in the LSU, the decoding of mRNAs is coupled with the peptide bond formation by the peptidyl transferase centre (PTC). During protein synthesis, mRNAs enter the ribosome through a tunnel in the SSU, are base paired with a tRNA and peptide bond formation is catalysed in the PTC of the LSU. The mRNA leaves the ribosome through an exit tunnel in the SSU, and the growing polypeptide chain emerges from the ribosome through a peptide exit tunnel (PET) in the LSU.

Advances in cryo-electron microscopy (cryo-EM) combined with X-ray crystallography allowed for atomic resolution views of prokaryotic and eukaryotic ribosomes (Ban et al., 2000; Ben-Shem et al., 2011; Klinge et al., 2011; Rabl et al., 2011; Wimberly et al., 2000; Yusupov et al., 2001). These high-resolution structures showed that the key functional centres of the ribosomal subunits (decoding centre and PTC) are evolutionarily conserved, as well as other important structural features such as the PET, the GTPase-activating centre (GAC) and the pseudoknot, which are necessary for translation fidelity. Despite this universal conservation of the functional core, the composition of eukaryotic ribosomes is much more complex than their bacterial counterparts, probably as a consequence of the translation process becoming more intricate throughout evolution.

In prokaryotes, the catalytically active ribosome exists as a 705 particle, named after its sedimentation coefficient in a sucrose density gradient. It contains three ribosomal RNAs (rRNA) and 33 ribosomal proteins (RPs). In comparison, mature eukaryotic ribosomes exist as 80 S particles containing a 40 S SSU and a 60S LSU. Ribosomal constituents are 79 (yeast)/80 (human) RPs and four rRNAs (25S (yeast)/28S (human), 18S, 5.8S and 5S) with eukaryote-specific extensions (reviewed in Melnikov et al., 2012) (Figure 1.1). The biogenesis of prokaryotic ribosomes involves a relatively small number of nonribosomal factors, whereas eukaryotes have developed a much more complex assembly process. 


\section{Introduction}

A

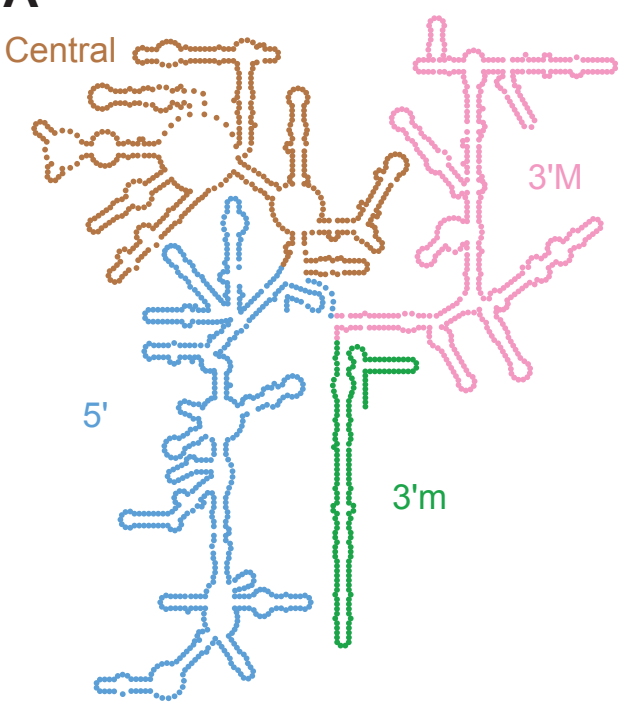

B

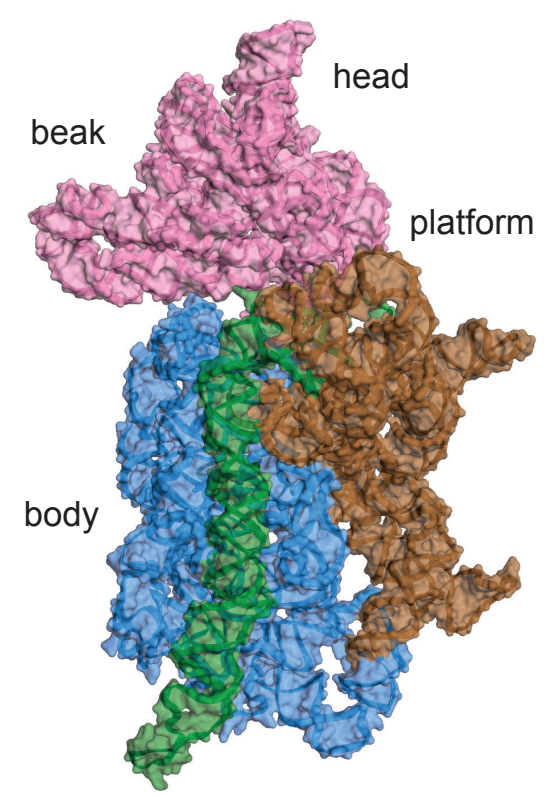

C

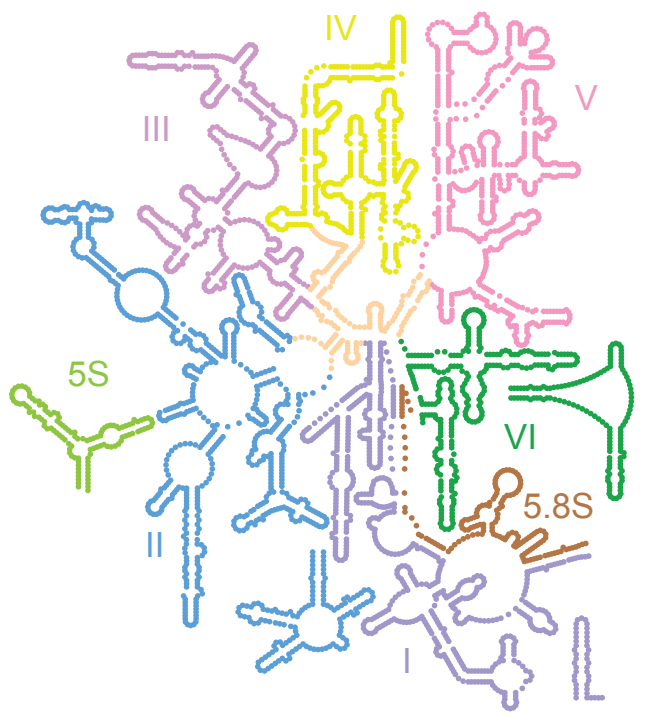

D

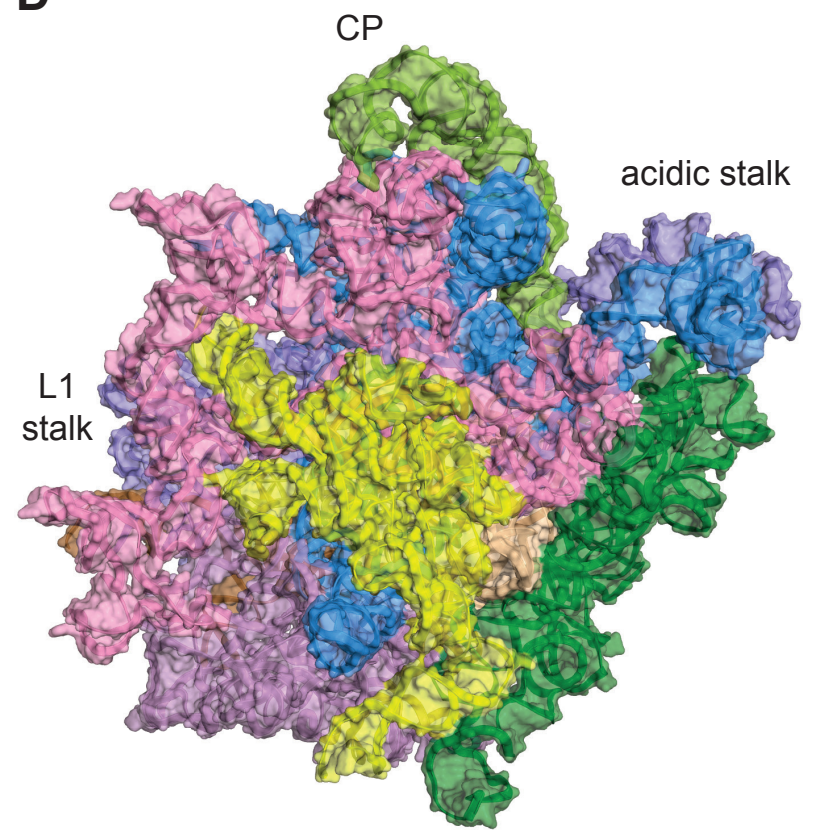

Figure 1.1 Secondary and tertiary structure of the S. cerevisiae rRNAs. (A) Secondary structure map showing the folding of the 18S rRNA into individual domains (Petrov et al., 2014). Domains are indicated in colours. 5': 5' domain (blue), central: central domain (brown), 3' M: 3' major domain, 3' m: 3' minor domain. (B) Crystal structure of the SSU rRNA (Ben-Shem et al., 2011). Secondary structure domains are represented following the same colour scheme as in (A). Conserved structural features are labelled (beak, body, head, platform). (C) Secondary structure map showing the folding of the 25S, 5.8S and 5S rRNAs (Petrov et al., 2014). The 5.8S rRNA and the 5S rRNA are indicated in brown and light green, respectively. The structural domains within the 25S rRNA are indicated in colours. I: domain I (dark purple), II: domain II (blue), III: domain III (light purple), IV: domain IV (yellow), V: domain V (pink), VI: domain VI (dark green). (D) Crystal structure of the LSU rRNAs (25S, 5.8S and 5S, Ben-Shem et al., 2011). Different rRNAs and secondary structure domains are represented following the same colour scheme as in (C). Conserved structural features are labelled (L1 stalk, CP, acidic stalk). 


\subsubsection{Ribosome biogenesis in yeast}

The synthesis of eukaryotic ribosomes is a very dynamic and energy consuming process (Figure 1.2). An actively growing yeast cell dedicates $60 \%$ of total transcription to rRNA and produces in turn 2,000 ribosomes per minute (Warner, 1999). The rRNAs and RPs are assembled in a process that span three subcellular compartments, and over 200 assembly factors (AFs) that transiently interact with the ribosomal precursors at various stages of their maturation, but are absent from the mature ribosomal subunits. For many of these AFs, the temporal and spatial order of events and precise molecular roles remain elusive.

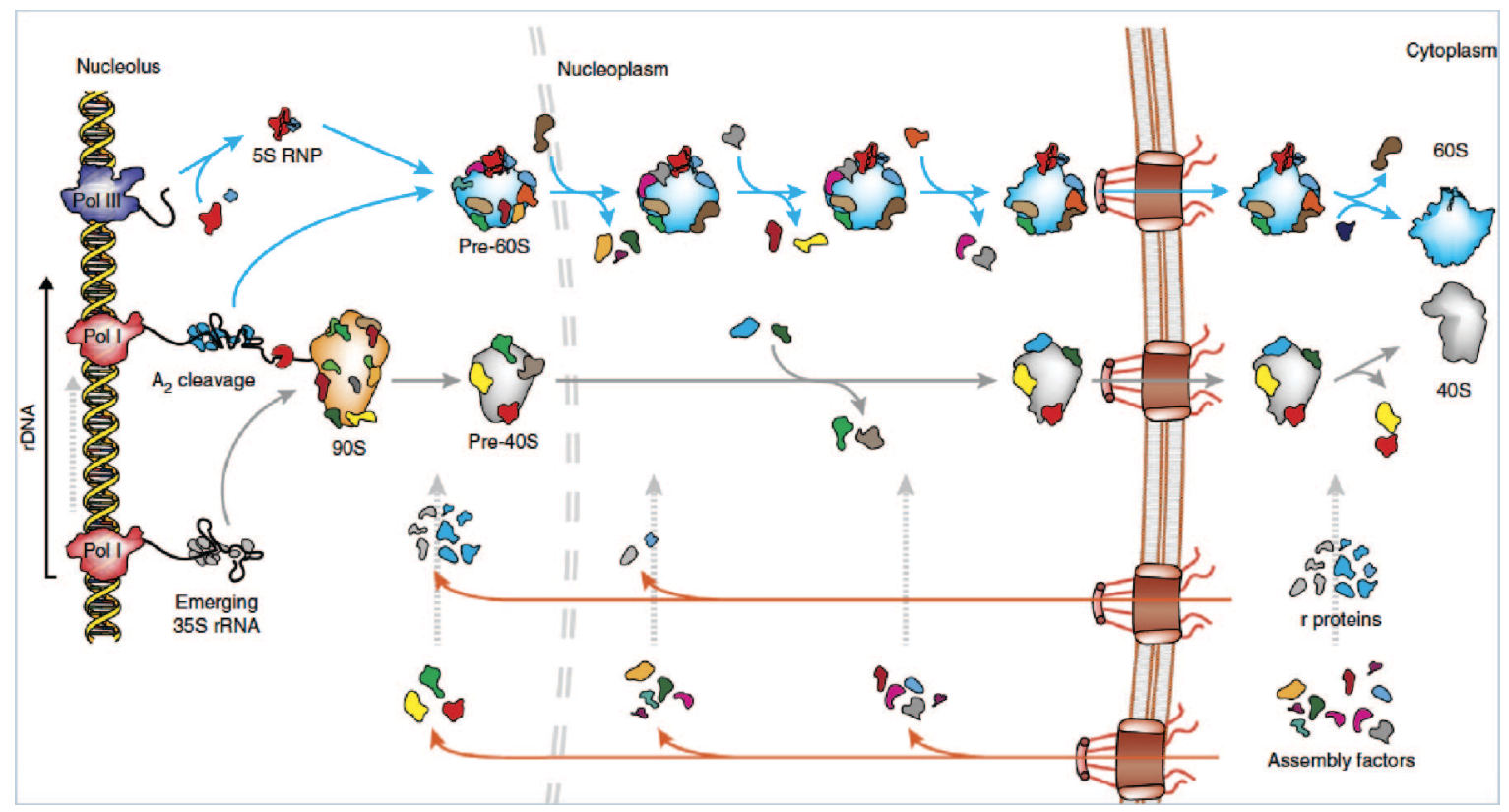

Figure 1.2 Eukaryotic ribosome assembly pathway. Schematic representation of eukaryotic ribosome biogenesis. Ribosomal DNA (rDNA) is transcribed by RNA polymerase I (Pol I) in the nucleolus to form a 35S precursor RNA. The nascent $35 \mathrm{~S}$ rRNA is subject to co-transcriptional processing ( $\mathrm{A}_{2}$ cleavage) and protein binding, leading to the formation of ribosomal precursors (90S, pre-40S, pre-60S). Independent maturation of small (40S) and large (60S) subunits occurs through three subcellular compartments (nucleolus, nucleus and cytoplasm) and involves sequential exchange of assembly factors and incorporation of ribosomal proteins (r proteins). The last maturation steps take place in the cytoplasm (Peña \& Hurt, 2017).

In Saccharomyces cerevisiae (yeast), ribosome biogenesis begins in the nucleolus with the transcription by RNA Polymerase I (Pol I) of the 150 rDNA tandem repeats (rDNA loci). Three of the four rRNAs (25S, $18 \mathrm{~S}$ and $5.8 \mathrm{~S}$ ) are co-transcribed as a $35 \mathrm{~S}$ pre-rRNA, together with two external transcribed spacers (5' and 3' ETS) and two internal transcribed spacers (ITS1 and ITS2), which are excised during the processing of the pre-rRNA 
(Figure 1.3). The $5 \mathrm{~S}$ rRNA is separately synthesised by RNA Polymerase III (Pol III) and later integrated into the pre-LSU.

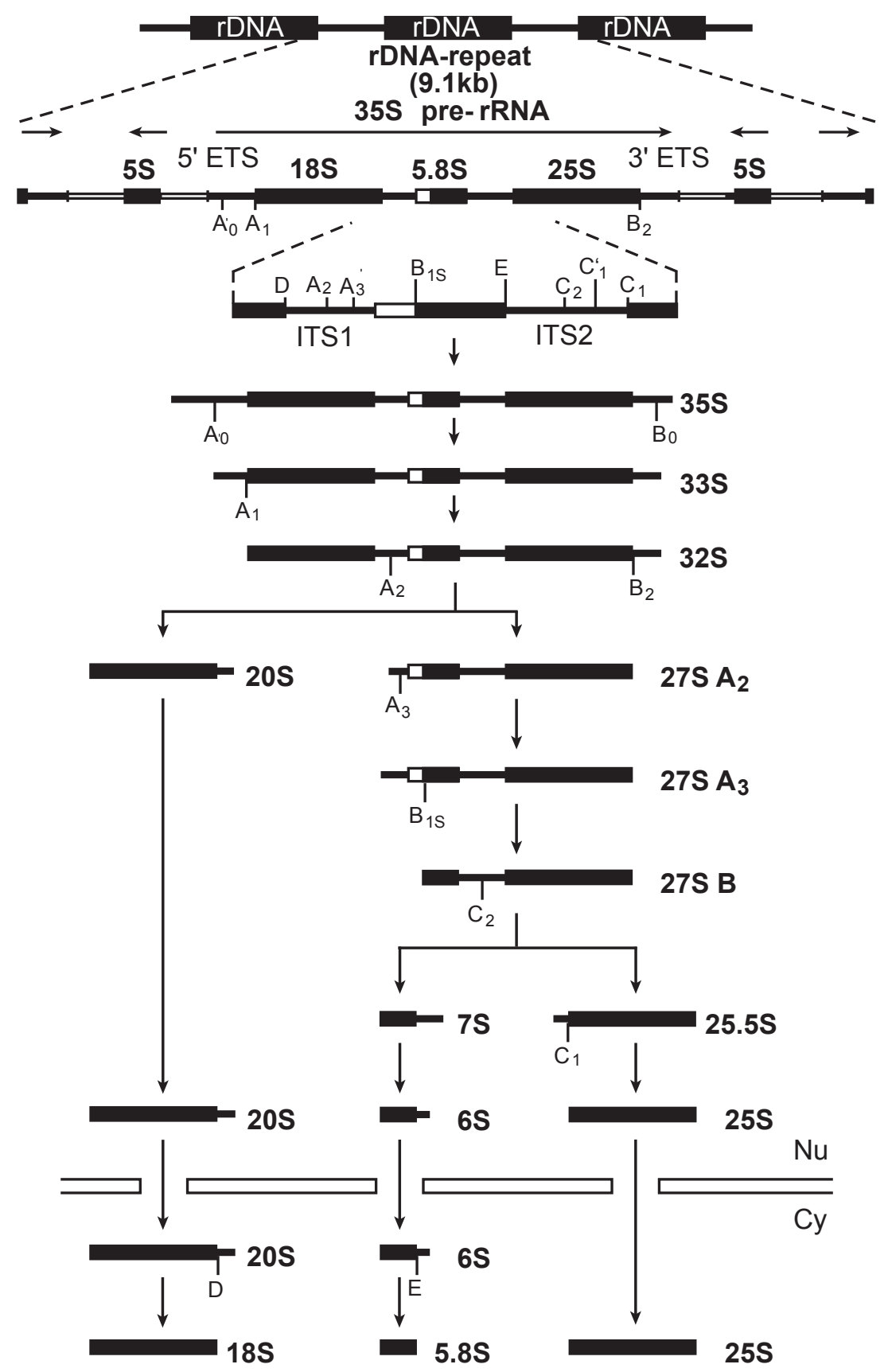

Figure 1.3 Pre-rRNA processing in yeast. Simplified schematic representation of the pre-rRNA processing pathway in yeast. Mature rRNAs and relevant rRNA precursors are represented with mature rRNA sequences depicted as black boxes, and pre-rRNA transcribed spacers as black lines. Specific pre-rRNA cleavage sites are indicated within the spacer's sequences. Processing steps taking place in the nucleus $(\mathrm{Nu})$ and the cytoplasm (Cy) are separated by white bars representing the nuclear membrane. Modified from Martin et al., 2013. 
As the 35S pre-rRNA is synthesised, early RPs and AFs are co-transcriptionally recruited to the nascent pre-rRNA to form the early precursors of the ribosomal subunits. Endonucleolytic cleavage of the pre-rRNA separates the precursors of the SSU (18S rRNA) and LSU (5.8S and 25S rRNA), which then follow distinct maturation pathways (Figure 1.3). Interestingly, while in higher eukaryotes pre-rRNA processing is almost exclusively post-transcriptional (Turowski and Tollervey, 2015), in yeast, these initial prerRNA cleavages can occur co- or post-transcriptionally (Koš and Tollervey, 2010; Osheim et al., 2004).

In the nucleolus and the nucleus, each subunit precursor undergoes extensive pre-rRNA processing and folding, together with pre-rRNA modification and RP incorporation. This maturation process is catalysed by the dynamic exchange of various AFs. For instance, different endo- and exonucleases are responsible for the ordered processing of the pre-rRNA, and small nucleolar RNAs (snoRNAs) guide chemical modifications of the rRNA sequences in the context of small nucleolar RNPs (snoRNPs). Additional modifications are introduced by methyltransferases and other modification enzymes, and together the rRNA modifications are thought to be important to fine-tune rRNA structure and function (Sharma and Lafontaine, 2015; Sloan et al., 2017; Watkins and Bohnsack, 2012). Moreover, dedicated chaperones and energy consuming proteins, such as GTPases, AAA-ATPases and RNA helicases, cooperate in the assembly (Kressler et al., 2012; Martin et al., 2013; Pillet et al., 2017). Importantly, the gradual incorporation of RPs, rRNA folding, and the chronological AF binding and release enable ribosome biogenesis to be an oriented process (Klinge and Woolford, 2019). During the maturation steps that take place in the nucleus, the pre-ribosomal particles gain export competence, and the association of a number of different export adaptors ensures their active export to the cytoplasm, where they undergo final maturation and quality control (Peña et al., 2017).

Given the importance of continuous ribosome production in growing cells, ribosome biogenesis is tightly regulated. For instance, the process is regulated by the availability of nutrients. The regulatory response to environmental changes is exerted at different levels: transcription of rDNA by Pol I, transcription of RP genes by Pol II, the activity of AFs, prerRNA processing and ribosome assembly (reviewed in de la Cruz et al., 2018). Importantly, while the primary regulator of ribosome biogenesis in eukaryotes is the Target of Rapamycin (TOR) pathway (Torreira et al., 2017; Vanrobays et al., 2008; Waliullah et al., 2017), ribosome biogenesis itself provides feedback regulation on the expression of RP and AF genes, that can ultimately lead to cell cycle arrest (Gómez-Herreros et al., 2013, 2017). 


\subsubsection{Small subunit assembly}

The mature SSU contains the 18S rRNA and $33 \mathrm{RPs}$, and is assembled in a fast, hierarchical manner. For this process, the spacer sequences flanking the mature 18S rRNA (5' ETS and ITS1) are important, as they are involved in the recruitment of ribosomal AFs, but also help to coordinate the assembly. Besides, structural subdomains within the 18S rRNA sequence bind different RPs and AFs as transcription progresses.

The SSU assembly starts co-transcriptionally in the nucleolus. During rDNA transcription, protein factors recruited to RNA elements start chaperoning and processing the nascent pre-rRNA. This co-transcriptional recruitment was initially visualised as so-called terminal knobs on chromatin spreads by electron microscopy (Miller and Beatty, 1969). Later, it was demonstrated that these structures are RNP complexes responsible for the compaction of the earliest $90 \mathrm{~S}$ pre-ribosomal particle on the nascent pre-rRNA (Mougey et al., 1993). The $90 \mathrm{~S}$ pre-ribosome or SSU processome is the earliest nucleolar SSU precursor and was defined as an RNP complex containing pre-rRNA sequences, some RPs, the U3 snoRNP and several AFs (Dragon et al., 2002). In recent years, available cryo-EM structures of the SSU processome from Saccharomyces cerevisiae and Chaetomium thermophilum, together with biochemical analyses, have allowed significant advances in understanding the architecture and the composition of this complex (Barandun et al., 2017; Kornprobst et al., 2016; Sun et al., 2017). For example, these studies showed that during yeast ribosome biogenesis, co-transcriptional processing of the pre-rRNA leads to the formation of a mature SSU processome carrying only the 18S rRNA precursor. As the 5' ETS sequence is transcribed, multiprotein complexes UtpA, UtpB, the U3 snoRNP and other protein factors are recruited to form a 5' ETS-containing particle (Chaker-Margot et al., 2015; Pérez-Fernández et al., 2007, 2011). Unlike most other snoRNPs that act in ribosome biogenesis, the U3 snoRNP does not mediate chemical modification of the rRNA sequence. Instead, this complex plays a key role in the assembly of the SSU processome, as the U3 snoRNA base pairs with both the 5' ETS and the 18S rRNA. These interactions determine the structural organisation of the SSU and are required for pre-rRNA cleavage at sites $A_{0}, A_{1}$ and $A_{2}$ (Figure 1.3, Kudla et al., 2011; Venema et al., 2000). The UtpA and UtpB complexes have a role in stabilising the 5' ETS particle, which serves as a platform for SSU subdomain maturation (Chaker-Margot et al., 2017; Hunziker et al., 2016; Sun et al., 2017).

Transcription progression through the 18S rRNA sequence induces the recruitment of domain-specific AFs. The gradual incorporation of RPs and multimodal binding proteins (such as Sas10 and Lcp5) at this stage prevents the premature formation or misfolding of tertiary structures (e.g. the pseudoknot that forms the core of the decoding centre) by 
restricting the conformational freedom of the pre-rRNA. Concurrent with the pre-rRNA folding, several snoRNPs introduce chemical modifications and the 18S rRNA subdomains (the 5', central, 3' major and 3' minor domains, Figure 1.1A and B) begin to be formed. Importantly, the spatial segregation of the rRNA domains allows their separate maturation within the SSU processome. Last, the external shell of the SSU processome is formed by AFs, including the RNA helicase and acetyltransferase Kre33, and the methyltransferase Emg1 (Barandun et al., 2018). Other AFs that are not present in the mature SSU processome structure might interact transiently with the particle, and their roles in the maturation process are less well understood (Klinge and Woolford, 2019).

After the partial maturation of the 18S rRNA domains, the transition from the SSU processome to a pre-40S particle requires the release of the 18S rRNA precursor from the 5' ETS-containing particle and the nascent pre-rRNA. RNA helicases, such as Dhr1, are necessary for the dissociation of the U3 snoRNA-5' ETS duplexes (Sardana et al., 2015; Zhu et al., 2016). Release of the U3 snoRNA enables processing of the pre-rRNA. The endonuclease Utp24 is thought to cleave sites $A_{1}$ (5' ETS) and $A_{2}$ (ITS1), giving rise to a 20 S pre-rRNA and releasing the 5' ETS (Wells et al., 2016). The nuclear exosome, recruited by exosome-interacting proteins in the particle, degrades the excised 5' ETS fragments and allows recycling of the associated AFs (Thoms et al., 2015). In the SSU processome, many AFs associate with domain-specific secondary structure elements and act as "place-holders" for other proteins. Their release allows for the incorporation of several RPs into the formed structures, thus producing a pre-40S particle in a nearly matured conformational state. In the nucleus, the pre-40S particles incorporate additional AFs important for export and later maturation steps. The translocation of SSU precursors from the nucleus to the cytoplasm occurs in a RanGTP- and Crm1-dependent manner. During maturation in the nucleus, the predicted nuclear export sequences (NES) of many AFs, such as Pno1, become accessible for binding of the export factor Crm1. Formation of an export complex with RanGTP directs the particle to the cytoplasm (Moriggi et al., 2014; Peña et al., 2017; Vanrobays et al., 2008).

Finally, maturation of pre-40S particles in the cytoplasm involves the release of the last AFs, functional proofreading of the subunit and processing of the 205 pre-rRNA resulting in the mature 18S rRNA. After removal of most of the AFs, the pre-40S particle transiently forms an 80S-like complex together with a mature 60S particle. This complex undergoes a non-productive cycle of translation, thereby testing the functionality of the pre-40S particle (Lebaron et al., 2012; Strunk et al., 2012). This "translation-like cycle" activates the cleavage of the pre-rRNA at site $D$ by the endonuclease Nob1 and the release of final AFs such as Pno1, Nob1 and Ltv1, producing mature SSU particles (Cerezo et al., 2019). 


\subsubsection{Large subunit assembly}

The yeast LSU contains three rRNAs (25S, 5.8S and 5S) and 46 RPs, and its assembly follows a more complex pathway than the SSU. As for the SSU, the assembly of this ribosomal subunit is linked to the stable association of RPs to key regions in the nascent pre-rRNA. Such key regions are six structural subdomains (domains I - VI, Figure 1.1C and D) of the 25S rRNA, which are important for forming LSU functional centres and for their maturation. Our current understanding of this complicated assembly process is based on biochemical and genetic studies of pre-rRNA processing and compositional analysis of pre-LSU particles together with the analysis of cryo-EM structures of different assembly intermediates. However, the architectures of early nucleolar particles remain elusive (Klinge and Woolford, 2019).

While the rDNA is being transcribed in the nucleolus, the nascent 25S rRNA sequence is extensively modified by snoRNPs. At the same time, the co-transcriptional binding of AFs directs and stabilises rRNA folding into a compact intermediate, which serves as a scaffold for further assembly. Interestingly, the LSU assembly begins prior to the cleavage events that separate the SSU and LSU maturation pathways. During the first folding events, the $5.8 S$ rRNA sequence is base paired with sequences in domains I and II of 25S rRNA, and ITS2 is restructured by the binding of early AFs. The proper folding of these domains serves as a checkpoint for LSU-assembly initiation by triggering $A_{2}$ cleavage and separation of the SSU and LSU processing pathways (Gamalinda et al., 2014; Turowski and Tollervey, 2015). Upon transcription termination, the 3' ETS is processed and the ITS1 cleaved at site $A_{3}$, resulting in the $27 S A_{3}$ pre-rRNA (Allmang and Tollervey, 1998). Subsequent processing of the 5 ' end of this pre-rRNA produces the 27SB pre-rRNA, containing the mature sequences of $5.8 \mathrm{~S}$ rRNA and $25 \mathrm{~S}$ rRNA separated by the ITS2.

During early maturation steps in the nucleolus, the $5 \mathrm{~S}$ rRNA is incorporated into the pre$60 S$ particles as the 5S RNP complex (5S rRNA, and RPs L5 and L11) forming the central protuberance. Also, the LSU functional centres (PTC, PET and GTPase activation centre) reach an almost mature conformation. The assembly of these structures requires both the entry and exit of AFs from the particle, many of which remain associated with the structured domains to prevent premature inter-domain interactions. The transition of pre-60S particles from the nucleolus to the nucleoplasm involves major structural remodelling. For instance, the formation of a functional PET upon exchange of AFs and the docking and rotation of the flexible central protuberance at the top of the particle serve as checkpoints before nuclear export. Importantly, structural remodelling and release of AFs bound near the ITS2 trigger the stepwise cleavage and processing of the spacer sequence. First, the endonuclease Las1 cleaves the 27SB pre-rRNA at site $C_{2}$, separating the $7 S$ and 
$25.5 \mathrm{~S}$ pre-rRNAs. Once in the nucleoplasm, the $25.5 \mathrm{~S}$ pre-rRNA is trimmed resulting in the mature 5' end of the 25S rRNA and components of the nuclear exosome process the 3 ' end of the 7S pre-rRNA to a 6 S pre-rRNA (Schuller et al., 2018; Thomson and Tollervey, 2010).

Prior to translocation to the cytoplasm, the functional centres of the ribosome undergo structural proofreading steps, which are coupled with export from the nucleus (Karbstein, 2013). For instance, the binding of the export factor Arx 1 serves as a checkpoint to test proper accommodation of RPs at the rim of the PET (Bradatsch et al., 2012). Similarly, the RNA helicase Dbp10 and a group of GTPases (including Nog2) influence structural maturation of the PTC. The subsequent release of Nog2 from the pre-ribosome enables binding of the AF Nmd3 and renders the particle export-competent (Ma et al., 2017; Matsuo et al., 2014). Interestingly, these events indicate that export of the relatively large preribosome relies on more than one transport factor for efficient translocation through the nuclear pore complex (NPC). The aforementioned Nmd3 factor is a NES-containing protein responsible for Crm1-dependent export, whereas other factors (such as Arx1) can directly interact with the NPC. Moreover, the Mex67-Mtr2 heterodimer contributes to the export in a RanGTP-independent manner (Nerurkar et al., 2015).

Final cytoplasmic maturation of the LSU involves the last step of pre-rRNA processing, the release of assembly and export factors and incorporation of the last RPs (Karbstein, 2013). The final step of pre-rRNA processing is the trimming of the $6 S$ pre-rRNA by the components of the exosome to form the mature 5.8S rRNA (Schuller et al., 2018; Thomson and Tollervey, 2010). At the same time, the release of AFs and their recycling is driven by the action of energy-consuming enzymes, such as Rlp24 release by the ATPase Drg1 (Kappel et al., 2012). The stepwise removal of factors bound to the functional centres enables assembly of RPs and licenses each domain to participate in protein synthesis (Klinge and Woolford, 2019; Lo et al., 2010).

\subsection{RNA helicases}

\subsubsection{Helicase structure and classification in families}

Many structured RNAs and RNPs carry out regulatory and catalytic functions in the cell and fulfil important roles in many processes of RNA metabolism, such as pre-mRNA splicing, translation and ribosome biogenesis. The function of these RNAs requires their correct folding in a hierarchical manner; formation of local secondary structures enables the formation of tertiary interactions between elements separated in sequence. Due to the low variety of bases in the RNA and the potential for non-canonical pairs beyond the 
Watson-Crick pairings, aberrant base-pairing interactions can occur in nascent RNAs, leading to the formation of non-native secondary structure elements. Moreover, the formation of RNPs relies on proper assembly of proteins onto structured RNA. Misfolded structures in RNAs and RNPs therefore require large-scale remodelling. RNA chaperoning proteins play key roles in restructuring misfolded structures, promoting the correct folding of RNA and enabling correct structural transitions (Herschlag, 1995; Jarmoskaite and Russell, 2014). Such chaperoning proteins include RNA helicases, a group of nucleoside triphosphate- (NTP-) dependent enzymes that bind or remodel structured RNAs and RNPs.

RNA helicases are ubiquitous proteins found in all three domains of life, also many viruses encode one or more members of this protein family. The comparison of sequence and structural features allowed the classification of helicases in superfamilies and families (Fairman-Williams et al., 2010). Most eukaryotic RNA helicases belong to the superfamily 2 (SF2). All SF2 helicases are characterised by a conserved helicase core harbouring the binding sites for NTP and RNA (Caruthers and McKay, 2002; Fairman-Williams et al., 2010; Jarmoskaite and Russell, 2014; Singleton et al., 2007). The helicase core consists of two tandem RecA-like domains (domain 1 and domain 2, Figure 1.4) in juxtaposition to each other and connected by a flexible linker sequence. The helicase core harbours up to 12 characteristic sequence motifs (Figure 1.4). These sequence motifs contact both ligands and, in the catalytic conformation of the helicase, are arranged to form an NTP. binding pocket and an RNA substrate tunnel.

Among the conserved motifs, all helicases carry the Walker A and Walker B motifs (motifs I and II, Figure 1.4), which are also found in many NTPases and coordinate the binding and hydrolysis of NTP (Walker et al., 1982). Besides motifs I and II, the motif VI is also required for NTP binding and hydrolysis. The motifs III and Va are suggested to couple the NTP binding and hydrolysis with the helicase activity (Pause and Sonenberg, 1992). Finally, motifs la-c, IV, IVa and V are implicated in contacting the substrate RNA (Caruthers and McKay, 2002). Importantly, residues of the conserved sequence motifs establish contacts primarily with the sugar-phosphate backbone of the substrate RNA, suggesting little sequence specificity for the substrate (Andersen et al., 2006; von Moeller et al., 2009; Tauchert et al., 2017). 
A

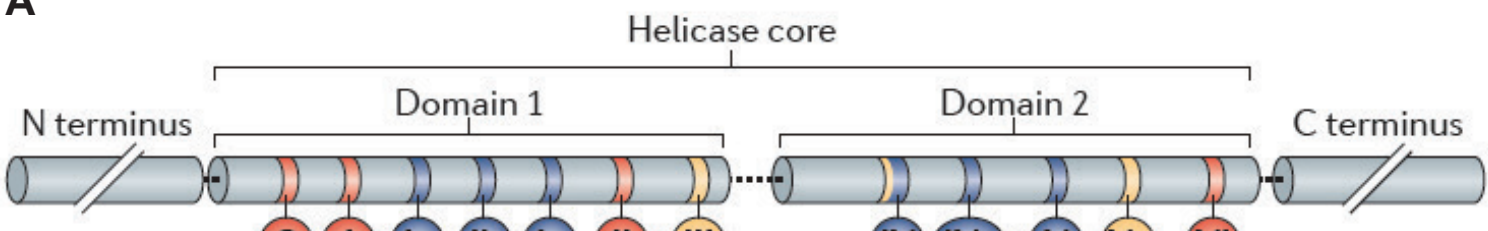

Q) (I) (Ia) (IC) (II) (II) (IV) IVa V (Va) (VI)

B

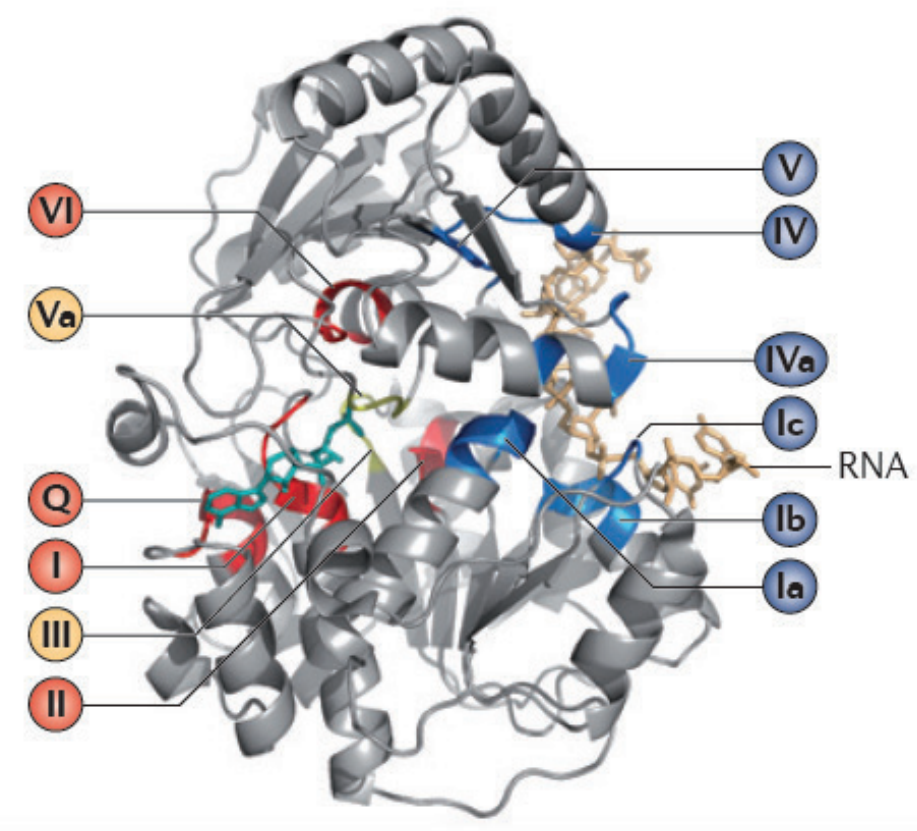

Figure 1.4: Organisation and structure of the helicase core of RNA helicases. (A) Schematic representation of the helicase core of RNA helicases and motif distribution in the two RecA-like domains (Domain 1 and Domain 2). Conserved signature motifs are colour coded according to their role in NTP binding (red), RNA binding (blue) or coupling NTP hydrolysis and helicase activity (yellow). (B) Structure of the helicase core of the Drosophila melanogaster DEAD-box protein Vasa. The conserved signature motifs are represented following the same colour scheme as in (A). Adapted from Linder and Jankowsky, 2011.

All these signature motifs are highly conserved within families of the SF2 superfamily. From the nine SF2 families, most RNA chaperones are found within the DEAD-box and DEAH-box proteins, named after the amino-acid sequence Asp-Glu-Ala-Asp (DEAD) or Asp-Glu-Ala-His (DEAH) of the Walker B motif, respectively (Linder et al., 1989). Interestingly, the molecular properties and mechanisms of these two helicase families appear to differ substantially from each other. For example, proteins of the DEAD-box family carry an extra characteristic sequence, besides the conserved motifs, consisting of an invariant glutamine, the Q-motif. This motif acts as a sensor for bound NTP and further regulates nucleotide hydrolysis (Cordin et al., 2004; Tanner et al., 2003).

Flanking the helicase core, RNA helicases often have amino- and carboxyl-terminal ( $\mathrm{N}$ - and $\mathrm{C}$-terminal) extensions variable in length and composition. While the DEAD-box 
family shows little conservation of these terminal regions, in the DEAH-box family the C-terminus is highly conserved and contains three defined domains: a winged helix (WH), a ratchet-like domain and an oligosaccharide binding $(\mathrm{OB})$ fold, important for helicase activity (Ozgur et al., 2015). In general, these ancillary domains are thought to be critical for the physiological specificity of helicases, serving as a platform for protein-protein interactions or mediating interactions with RNA substrates (Jarmoskaite and Russell, 2014; Ozgur et al., 2015).

\subsubsection{Mechanism of action}

In all catalytic RNA helicases, the helicase core couples NTP-binding and RNA-dependent hydrolysis to protein conformational changes. For many helicases, these conformational changes lead to the disruption of RNA base pairs and unwinding of duplex RNA, the bestknown molecular function of these enzymes. However, it has been demonstrated that RNA helicases can also act by annealing RNA strands, remodelling RNPs and serving as RNAclamps (Linder and Jankowsky, 2011; Semlow et al., 2016). All of these roles require similar steps of NTP and RNA binding and NTP hydrolysis, but regulation of the hydrolysis cycle can modulate the way the RNA helicase functions.

Structural and biochemical experiments have revealed the conformational changes that RNA helicases undergo during catalytic cycles, and showed that proteins of the DEADbox and DEAH-box families work by remodelling RNA following two distinct mechanisms (reviewed in Gilman et al., 2017; Jarmoskaite and Russell, 2014; Ozgur et al., 2015).

DEAD-box proteins function primarily as adenosine triphosphate- (ATP-) driven, non-processive helicases that bind and unwind short RNA helices by forcing local RNAduplex separation (Yang et al., 2007a). Structural analyses of different DEAD-box proteins revealed that prior to ATP or RNA substrate binding, the two RecA-like domains remain ordered but spatially separated from each other forming a cleft (open conformation) (Mallam et al., 2012). ATP initially binds to the ATP-binding pocket in D1 formed by the $\mathrm{Q}$ motif, and double-stranded RNA (dsRNA) binds to both RecA-like domains (Mallam et al., 2012; Samatanga and Klostermeier, 2014), which ultimately leads to the formation of a ternary complex of the enzyme, containing ATP and dsRNA. Formation of inter-domain contacts between amino acids from the helicase motifs induces the closure of the complex, with both RecA-like domains coming together and creating a functional ATPase active site (ON state) (Hilbert et al., 2009). These conformational changes distort the bound RNA, producing a kink in one of the RNA strands retaining it in a tight complex with the enzyme, while the other strand is released from its base-pairing interactions and the complex. 
Importantly, RNA unwinding precedes ATP hydrolysis, which is only required for release of the products and returning the helicase into an open conformation (OFF state). ATP hydrolysis results in the release of inorganic phosphate $(\mathrm{Pi})$ and the efficient release of the tightly bound RNA strand (Liu et al., 2008). Moreover, complete unwinding of an RNA helix is achieved in a single cycle of ATP binding and hydrolysis (Chen et al., 2008). Interestingly, using a similar mechanism as unwinding, some DEAD-box helicases can function as effective nucleotide-dependent RNA clamps. By regulating steps of the ATPase cycle, the helicase core can be trapped in a closed RNA-bound state forming stable, long-lived complexes with RNA. For example, it has been shown that the RNA helicase Ded1 can form long-lived complexes with RNA when ATP hydrolysis is impeded (Linder and Jankowsky, 2011; Liu et al., 2014a; Nielsen et al., 2008). Moreover, DEADbox helicases can remove proteins from structured and unstructured RNAs in an ATP-driven manner that does not require translocation and unwinding, thus further expanding the molecular functions of these enzymes (Bowers, 2006; Fairman, 2004). For example, RNA helicase Dbp5 is proposed to remove export factor Mex67 from mRNA during mRNA export (Lund and Guthrie, 2005).

Despite the sequence and structural similarities with DEAD-box proteins, DEAH-box helicases have a very different mechanism of action. Unlike DEAD-box proteins, DEAHbox helicases are able to bind and hydrolyse all four NTPs, and typically require a 3' singlestranded RNA (ssRNA) extension adjacent to the RNA duplex for loading onto the RNA. These enzymes are thought to mediate duplex unwinding by translocating along a ssRNA while displacing the complementary strand (Tauchert et al., 2017). Importantly, crystal structures show that the conserved C-terminal domains of these enzymes interact with the helicase core forming an RNA-binding tunnel (He et al., 2010; Walbott et al., 2010). Initially, binding of NTP induces large rearrangements of the C-terminal domains, leading to the opening of the RNA-binding tunnel. This step is key for enabling binding of DEAHbox proteins to sSRNA regions within folded RNA substrates in the cell. Binding to RNA and NTP induces the closure of the helicase core, trapping the bound RNA between D1 and D1. Hydrolysis of NTP promotes disruption of nucleotide-mediated interactions between D1 and D2, as well as contacts between D2 and the RNA. Along with these conformational transitions, movement of the helicase core domains relative to the trapped RNA strand results in the directional translocation of the enzyme (He et al., 2017). Upon NTP release, the helicase is returned to an open conformation and D2 is able to establish new interactions with the RNA. A new cycle of NTP binding and helicase core closure leads to the translocation of the helicase by one nucleotide. The RNA helicase toggles between open and closed conformation upon continuous NTP hydrolysis, thus 
translocating in 3'-5' direction along a ssRNA (Hamann et al., 2019; He et al., 2017; Tauchert et al., 2017). Interestingly, it has been proposed that these RNA helicases can remodel RNPs by pulling on RNA strands rather than translocating through them, thus disrupting base pairs in a process termed winching (Semlow et al., 2016).

Besides these mechanisms of action, many RNA helicases do not only catalyse the unwinding of RNA duplexes but can also promote RNA rearrangements by annealing RNA strands (Halls et al., 2007; Linder and Jankowsky, 2011; Uhlmann-Schiffler, 2006; Yang et al., 2007b). A prominent example is the DEAD-box helicases Ded1, which has been proposed to anneal RNA strands during cytoplasmic translation in an ATP-independent manner (Yang and Jankowsky, 2005).

\subsubsection{Regulation of RNA helicase activity}

Besides the relative promiscuity that arises from lacking RNA sequence specificity, RNA helicases possess inherently low catalytic activity. This is mostly due to intrinsic structural features that enable their autoregulation, such as interactions within the RecA-like domains or ancillary regions in the $\mathrm{N}$ - and $\mathrm{C}$-terminus (reviewed in Ozgur et al., 2015). Modulating the ATPase and unwinding activity and/or the substrate specificity of these proteins is essential for temporal and spatial regulation of RNA helicases, and different mechanisms of regulation have been proposed so far (Sloan and Bohnsack, 2018).

\subsubsection{RNA helicase regulation by cofactor proteins}

The action of some RNA helicases of the DEAH-box family can be modulated by protein partners containing a glycine-rich domain (G-patch domain). The conserved OB fold in the C-terminus of DEAH-box helicases is key for mediating the interaction with the inherently disordered G-patch domain (Aravind and Koonin, 1999; Robert-Paganin et al., 2015). To date, five G-patch proteins have been identified in yeast: Pfa1, Gno1, Ntr1, Cmg1 and Spp2; and they all act as positive regulators of DEAH-box helicases. Notably, the multifunctional helicase Prp43 (DHX15 in human) interacts with four of the five G-patch proteins. Binding of the different G-patch proteins to Prp43 leads to stimulation of the ATPase and/or unwinding activity of the helicase. Moreover, it has been shown that the interaction of the different G-patch proteins with Prp43 is mutually exclusive and that the individual cofactors target the helicase to its different cellular functions (Heininger et al., 2016). The fifth G-patch protein, Spp2, interacts with the RNA helicase Prp2, which is involved in pre-mRNA splicing, and stimulates its ATPase activity (Warkocki et al., 2015). 
Interestingly, homologues of the yeast G-patch proteins are present in higher eukaryotes, and many interactions with their cognate RNA helicases are evolutionarily conserved.

Importantly, many additional G-patch proteins are expressed in humans, suggesting an expanded network of RNA helicase regulation by G-patch proteins compared to yeast. For instance, the catalytic activity of DHX15 can be stimulated by the nucleolar G-patch protein NFkB-repressing factor (NKRF), and it has further been shown that these proteins function together in ribosome biogenesis (Memet et al., 2017). Although the mechanism of DEAH-box helicase regulation by G-patch proteins remain poorly understood, it has been suggested that G-patch protein binding might induce conformational changes that relieve helicase autoinhibition and help to coordinate ATP hydrolysis and unwinding activity (Christian et al., 2014; Robert-Paganin et al., 2017; Tauchert et al., 2017).

Another prominent group of proteins that regulate RNA helicases are the MIF4G domaincontaining proteins. All these proteins contain the conserved middle domain of elF4G (MIF4G domain) consisting of ten antiparallel $\alpha$-helices organised as five HEAT repeats (named after four proteins that carry such structure: ㅂuntingtin, elongation factor 3, protein phosphatase $2 \underline{A}$ and yeast kinase $\underline{T} O R 1)$. These structures form an arc with concave and convex surfaces (Ponting, 2000). To date, 12 MIF4G domain-containing proteins have been identified in humans, and seven (Tif4631, Tif4632, NMD2, Gle1, Sto1, Sgd1, Cwc22) in yeast. The MIF4G domain-containing proteins interact with proteins of a subfamily of the DEAD-box helicases, termed the elF4A-like helicases. These so-called "minimal helicases" consist of only a helicase core and lack $\mathrm{N}$ - and C-terminal extensions, similar to the translation initiation helicase elF4A (elF4A-I/II in humans) after which the group has been named (Andreou and Klostermeier, 2013).

Interestingly, MIF4G domain-containing proteins have been suggested to not only stimulate the catalytic activity of elF4A-like proteins but also, in some cases, to inhibit it. Structural and biochemical studies of DEAD-box helicase-MIF4G domain-containing protein complexes allowed for a molecular understanding of these two mechanisms of regulation. The best-studied example of helicase activation by an MIF4G domaincontaining protein is that of elF4A and elF4G (Schutz et al., 2008). It has been shown that the MIF4G domain of elF4G interacts with both RecA-like domains of elF4A, leading to a "half-open" conformation. This conformation promotes nucleotide and phosphate release, thus allowing a rapid switch between the open and closed conformations that, in turn, leads to increased catalytic activity of the helicase (Garcia-Garcia et al., 2015; Hilbert et al., 2011). In contrast, the interaction of CWC22 with the RNA helicase elF4A-III/DDX48 via its MIF4G domain blocks the helicase in an inactive, closed conformation, which is the state in which it is required during assembly of exon-junction complexes (EJCs; 
Steckelberg et al., 2012). It has been proposed that in the elF4A-III/CWC22 complex, an extra $\alpha$-helix in the C-terminus of the MIF4G domain promotes the reorientation of the elF4A-III RecA-like domains, pulling the RNA binding residues apart thereby disrupting the ATP binding pocket. This conformation leads to inactivation of the helicase and enables it to act as an RNA clamp by ensuring a stable grip on the substrate RNA (Buchwald et al., 2013; Steckelberg et al., 2012). Interestingly, elF4A-III is a multifunctional RNA helicase that performs different molecular functions and interacts with different MIF4G domaincontaining proteins in the context of EJC, ribosome biogenesis and translation initiation (Alexandrov et al., 2011; Choe et al., 2014; Steckelberg et al., 2012). This suggests that MIF4G domain-containing proteins could recruit DEAD-box helicases to different pathways and induce switching of molecular functions by modulating the catalytic activity of the RNA helicase. Importantly, all the available data suggests a mechanism of regulation by MIF4G domains that can fine-tune their specificity to DEAD-box helicases by subtle changes in intermolecular connections, resulting in inhibition or activation of the helicase activity.

Besides these two main groups of RNA helicase cofactors, many RNA helicases interact with and are regulated by dedicated protein-cofactors that do not belong to a cofactor family. In many cases, the mechanism of regulation used by these RNA helicase/cofactor pairs involves conformational regulation, as for DEAD-box helicases and MIF4G domaincontaining proteins. For example, the interaction of elF4A-III with the EJC components MAGO and Y14 stabilises a closed conformation and promotes phosphate release (Ballut et al., 2005). Other cofactors modulate the activity of RNA helicases by altering their interactions with RNAs, either by recruiting the helicase to its target RNA or by sterically blocking the access of RNA to the helicase (von Moeller et al., 2009; Montpetit et al., 2011; Noble and Song, 2007; Sharif et al., 2013). However, for most stand-alone helicasecofactor pairs, the mechanisms of regulation remain unknown.

\subsubsection{Non-cofactor mediated mechanisms of RNA helicase regulation}

Despite the overall lack of sequence specificity of the helicase core, some RNA helicases are recruited to RNA substrates either by secondary structure recognition or by sequencespecific recruitment. For example, the RNA helicase DbpA from E. coli carries a C-terminal RNA binding domain (RBD) that mediates specific binding to a hairpin within the 23S rRNA sequence (Diges, 2001; Rudolph and Klostermeier, 2015). Also, a sequence-specific binding mechanism has been proposed for the DEAD-box helicase DDX17, which is able to bind a subset of primary micro RNAs (pri-miRNAs) that contain a VCAUCH motif (Mori et al., 2014). In contrast, many RNA helicases are targeted to their RNA substrates by 
ancillary domains in a non-sequence-specific manner. One reported example is the yeast DEAD-box helicase Mss116, which contains an unstructured basic C-terminal tail, which is implicated in the binding of structured RNAs. This basic tail serves as a functional tether, establishing electrostatic interactions with exposed helices and anchoring the helicase in proximity to its substrate duplexes (Mallam et al., 2011). Ancillary domains of RNA helicases also play pivotal roles in viral RNA recognition, as best exemplified by the RIGI-like helicases. These helicases possess two distinct auxiliary protein domains that mediate RNA binding; the specialised insertion domain HEL2i located between the RecAlike domains promotes recognition of dsRNA, whereas the C-terminal regulatory domain recognises and interacts with specific viral RNA features (Rawling and Pyle, 2014). Similarly, the RNA helicase YTHDC2 is recruited to a subset of chemically modified transcripts by a YTH domain that specifically binds to RNAs containing $N^{6}$-methyladenosines ( $\mathrm{m}^{6} \mathrm{As}$ ) (Kretschmer et al., 2018; Wojtas et al., 2017).

In recent years, non-coding RNAs (ncRNAs) have emerged as new players in RNA helicase regulation. In some cases, binding of a ncRNA modulates the catalytic activity of a helicase in a similar way to a protein cofactor. For example, the long non-coding RNA (IncRNA) BC1 contributes to translational control in dendritic cells by modulating the activity of the elF4A-I helicase; association of the IncRNA specifically blocks the RNA unwinding activity of elF4A-I and simultaneously stimulates its ATPase activity (Lin et al., 2008). An alternative mechanism for RNA helicase regulation by ncRNAs has been proposed based on the IncRNA BC200 and the helicase DHX36 (Booy et al., 2016). Here, the IncRNA associated with the helicase is suggested to act as an acceptor of the unwound single-stranded RNA products, enhancing duplex separation by hindering reannealing during progressive cycles of unwinding (Booy et al., 2016). In addition, posttranslational modification (PTM) can dynamically modulate the activity of RNA helicases. High-throughput screens revealed that many RNA helicases are indeed modified by phosphorylation, methylation, acetylation, ubiquitination and/or sumoylation (Gustafson and Wessel, 2010; Hornbeck et al., 2015; Liu et al., 2014c), and in many cases, these PTMs have an impact on the cellular functions of the helicases. For instance, the sumoylation of DDX5 enhances its transcriptional repression activity, and extensive phosphorylation of DHX3X is required for inducing an innate immune response (Jacobs et al., 2007; Soulat et al., 2008). Moreover, some PTMs directly alter the catalytic activity of RNA helicases. For example, acetylation and de-acetylation of three lysine residues of the multifunctional DEAD-box helicase DDX21 modulate its unwinding activity, which is essential for its role in safeguarding genome integrity (Song et al., 2017). 


\subsubsection{Biological functions}

RNA helicases are required in every step of RNA metabolism and are important drivers and regulators of gene expression (Bourgeois et al., 2016; Jarmoskaite and Russell, 2014). Their wide range of molecular functions is key for these enzymes to perform different roles in different pathways. Although many RNA helicases are required for specific processes, several of these enzymes act in more than one process, i.e. the helicases Prp43/DHX15 (ribosome biogenesis, pre-mRNA splicing) and elF4A-III (ribosome biogenesis, EJC assembly, translation initiation). These proteins generally function within larger multicomponent assemblies, such as the spliceosome, the translation initiation machinery or the nascent pre-ribosomal subunits. Ribosome biogenesis is the pathway that involves the most RNA helicases, with a total of 21 in yeast and even more in humans. Within this process, helicases modulate the dynamics of snoRNAs and/or promote structural changes within the ribosomal subunit precursors (detailed in section 1.3). Pre-mRNA splicing is another cellular process that requires many RNA helicases. In yeast, eight RNA helicases participate in pre-mRNA splicing; three DEAD-box helicases and one Ski2-like helicase are required for spliceosome assembly, and four DEAH-box helicases are required for the catalytic steps of the splicing reactions. These RNA helicases have been implicated in many different molecular functions (such as structural rearrangements, unwinding of small nuclear RNAs (snRNA) and snRNA-pre-mRNA duplexes, protein displacement), and their highly regulated mode of action provides directionality to the splicing process (Jarmoskaite and Russell, 2014). For example, the DEAD-box helicase Sub2 (UAP56 in humans) is suggested to displace Mud2 (U2AF65 in humans) from pre-mRNAs in early stages of spliceosome assembly (Kistler, 2001; Shen et al., 2008), whereas the DEAH-box protein Prp22 (DHX8 in humans) disrupts base pairing between the U5 small nuclear RNA and the pre-mRNA, leading to the release of the mRNA (Schwer, 2008). Interestingly, besides its roles in ribosome biogenesis (see below), the multifunctional DEAH-box helicase Prp43 mediates the release of the intron lariat and spliceosome disassembly (Fourmann et al., 2013). Additionally, RNA helicases can act as proof-readers of the splicing process, distinguishing between correct and incorrect products and promoting the dissociation of aberrant ones (Semlow and Staley, 2012). In mitochondrial RNA metabolism, several autocatalytic RNAs require RNA helicases Mss116 (S. cerevisiae) and CYT19 (Neurospora crassa) for adapting their native conformations. These helicases then act as chaperones, for example, annealing RNA strands to facilitate folding (Fedorova et al., 2010; Karunatilaka et al., 2010).

Many RNA helicases act as regulators of transcription, either as activators or repressors (Fuller-Pace, 2013). The best-described example is the mammalian DEAD-box protein 
DDX5 and its paralogue DDX17, which interact with transcription factors and can modulate their activity (Xing et al., 2019). Interestingly, the yeast homologue of these helicases (Dbp2) has been proposed to promote efficient assembly of mRNA-binding proteins, and to resolve RNA-DNA hybrids in nascent RNAs (Ma et al., 2016; Tedeschi et al., 2018). Many RNA helicases are also required for the RNP remodelling steps involved in mRNA export. For example, the DEAD-box helicase Dbp5 mediates the dissociation of the export factor Mex67 from exported mRNPs at the rim of the nuclear pore complex, a critical step during mRNA export (Lund and Guthrie, 2005; Tran et al., 2007). Cytoplasmic translation of mRNAs also requires the action of many RNA helicases, for example, to perform rearrangements of structures within the 5' UTR during cap-dependent translation initiation. A well-studied example is the DEAD-box helicase elF4A (elF4A-I/II), which is proposed to promote loading of the pre-initiation complex onto the mRNA by unwinding secondary structures in the 5' UTR (Aitken and Lorsch, 2012; Svitkin et al., 2001). Other helicases are involved at later stages of translation; for example, Dbp5 (DDX19B in humans) is required for the recruitment of release factors elRF1 and eRF3 to translation termination complexes (Gross et al., 2007; Tieg and Krebber, 2013). Furthermore, RNA helicases also contribute to cytoplasmic mRNA degradation (Hardwick and Luisi, 2013). The DEAD-box helicase Dhh1 (DDX6 in humans) promotes mRNA decapping by restructuring the mRNA 5' end (Fischer, 2002), whereas Dbp2 modulates nonsense mediated decay in humans and yeast (Bond et al., 2001; Geißler et al., 2013). Many RNA helicases are important for RNA granule dynamics, where they contribute to the crosstalk between mRNA storage, translation and decay (Hooper and Hilliker, 2013). For instance, the ATPase activity of the DEAD-box helicase Dhh1 regulates the dynamics of processing bodies (PB) formation. Furthermore, stimulation of the ATPase activity by the MIF4G domain-containing protein Not1 is essential for promoting disassembly of PBs. It is thereby proposed that PB formation in response to stress is controlled by regulation of the interaction between Dhh1 and the MIF4G domain-containing protein Not1 (Mugler et al., 2016).

Additionally, these RNA helicases have been shown to play roles in (miRNA) biogenesis, as, for example, DDX5 and DDX17 are required for maturation of some pri-miRNAs (Mori et al., 2014; Motiño et al., 2015). RNA helicases are also involved in viral RNA sensing and stimulation of the subsequent immune response, as has been proposed for the DEAHbox helicases DHX33 and DHX9 (Liu et al., 2014b; Zhang et al., 2011). 
Introduction

\subsubsection{RNA helicases and their relation to diseases}

In line with their central roles in many essential cellular processes, RNA helicases have been implicated in many different diseases, including viral infection, neurological diseases and cancer. For instance, as $80 \%$ of all viruses are RNA viruses and many lack RNA helicases of their own, several host RNA helicases can be hijacked during the course of viral infections (Meier-Stephenson et al., 2018). For example, the HIV-I virus recruits cellular helicases such as DDX1 to support viral replication (Edgcomb et al., 2012; Krishnan and Zeichner, 2004). Interestingly, many RNA helicases also participate in the immune response against viral infections, such as RIG-I, which has been proposed to sense viral RNA and trigger the innate immune response (Bruns and Horvath, 2012; Rawling and Pyle, 2014). Moreover, mutations, as well as dysregulation of several RNA helicases, have been shown to have a role in cellular transformation in cancer (Abdelhaleem, 2004; Steimer and Klostermeier, 2012). Prominent examples are the translation initiation helicases, such as elF4A, that mediate the translation of oncogenic mRNAs with complex 5' UTR structures (Heerma van Voss et al., 2017). Other RNA helicases, such as DDX5, have been suggested to activate oncogenic transcription factors (Clark et al., 2013). Importantly, RNA helicases play additional roles in cancer development through, among other processes, alternative pre-mRNA splicing, miRNA regulation, ribosome biogenesis and apoptosis (Clark et al., 2008; Fuller-Pace, 2013; Suzuki et al., 2009). Furthermore, correct RNA processing plays a key role in the nervous system, and RNA helicases involved in pre-mRNA splicing and RNA editing (such as UAP56 and DDX19) have been linked to neurodegenerative disease (Steimer and Klostermeier, 2012). Given the extensive link between RNA helicases and diverse diseases, these enzymes represent potential targets for therapeutic applications.

\subsection{RNA helicases in ribosome biogenesis}

In yeast 21 predicted RNA helicases have been reported so far to be required for ribosome biogenesis. Early studies allowed their classification in LSU and SSU RNA helicases, according to affinity purifications of pre-ribosomal complexes and analyses of pre-rRNA processing defect upon depletion or mutation of individual RNA helicases. So far, eight RNA helicases (Dhr1, Dhr2, Dbp4, Dbp8, Fal1, Rok1, Rrp3 and Kre33) are implicated in small subunit maturation, ten RNA helicases (Dbp2, Dbp3, Dbp6, Dbp7, Dbp9, Dbp10, Drs1, Mak5, Spb4 and Mtr4) in large subunit maturation and three (Prp43, Has1 and Dbp5) are required for the maturation of both subunits (Martin et al., 2013). All these enzymes, with the exception of Dbp2, Dbp3 and Dbp7, are essential for cell viability, suggesting they 
carry out unique non-compensable functions in the cell. To date, the molecular functions of most RNA helicases in ribosome biogenesis remain unclear. However, functional roles have been proposed for some of these enzymes in modulating the dynamics of snoRNPs on pre-ribosomes and driving structural remodelling of the pre-ribosomal subunits. Identifying the binding sites of RNA helicases on the pre-rRNA is key to studying their molecular functions. In recent years, the development of crosslinking and sequencing techniques (Bohnsack et al., 2012; Granneman et al., 2009) made it possible to determine the binding sites of some RNA helicases on the pre-rRNAs. These results, together with the recently available structures of pre-ribosomal particles (Barandun et al., 2017; Bradatsch et al., 2012; Kater et al., 2017; Wu et al., 2016; Zhou et al., 2019), as well as the mature ribosome (Ben-Shem et al., 2011), are important tools for studying the role of RNA helicases during ribosome biogenesis.

\subsubsection{The roles of RNA helicases in regulating snoRNA dynamics on pre- ribosomes}

During ribosome biogenesis, many snoRNAs base-pair with the pre-rRNAs and guide sitespecific modifications (methylation, pseudouridylation or acetylation) within sequences that ultimately form functionally important regions of the ribosome. Interestingly, some snoRNAs instead contribute to pre-rRNA folding (Watkins and Bohnsack, 2012). There are 75 snoRNAs in yeast, three of which (U3, U14 and snR30) are essential. As snoRNAs form stable interactions with pre-rRNAs, their release is important for ongoing ribosome biogenesis and, in some cases, this dissociation might require assistance from protein factors. Quantitative screening of snoRNA levels on pre-ribosomes upon RNA helicase depletion lead to proposed roles for some proteins of this family in promoting the dissociation as well as the binding of snoRNAs (Bohnsack et al., 2008). Importantly, RNA helicases can likely have direct or indirect roles in promoting snoRNA release. Given the unwinding function of RNA helicases, it is likely that some can promote the release of snoRNAs by directly unwinding the snoRNA-rRNA base-pairing. Such a model has been proposed for the DEAD-box helicase Rok1, which is required for release of the snR30 snoRNA from pre-ribosomes (Bohnsack et al., 2008). UV-crosslinking and analysis of cDNA (CRAC) results showed that Rok1 binds to snR30 as well as its known binding site in the pre-rRNA. Further analysis of the CRAC data revealed hybrid snoRNA-rRNA sequences crosslinked to Rok1, suggesting a direct role of the helicase in unwinding the snoRNA-rRNA duplex (Martin et al., 2014). Such a direct model of snoRNA release has also been proposed for the DEAH-box helicase Prp43, which is required for release of a subset of snoRNAs from pre-ribosomes (Bohnsack et al., 2009). Similarly, Prp43 binds to 
snoRNAs and their cognate binding sites on the pre-rRNA, and the catalytic activity of the helicase is required for efficient snoRNA release (Bohnsack et al., 2009). Besides enabling the release of snoRNAs, Prp43 is also required for promoting the incorporation of the snoRNAs snR64 and snR67 into pre-ribosomes, probably by remodelling pre-rRNA secondary structures and thus enabling snoRNA-pre-rRNA base-pairing (Bohnsack et al., 2009). For other RNA helicases, the mechanism by which they mediate snoRNA release is less clear and might involve structural remodelling of the pre-rRNA rather than unwinding of snoRNA-pre-rRNA duplexes. For example, while CRAC data suggests that the DEADbox helicase Has1 directly unwinds the U14 snoRNA (Bohnsack et al., 2008; Brüning et al., 2018), depletion of the DEAD-box helicase Dbp4 also leads to an indirect accumulation of U14 on pre-ribosomes (Koš and Tollervey, 2005; Soltanieh et al., 2015). Moreover, the DEAH-box helicase Dhr1 is implicated in the dissociation of the U3 snoRNA from the pre18S rRNA precursor to allow the formation of the pseudoknot structure, a function that has been demonstrated to be conserved in humans (Choudhury et al., 2019; Sardana et al., 2015). Interestingly, the acetyltransferase-helicase Kre33 is thought to facilitate annealing of the snoRNAs snR4 and snR45 around Kre33 acetylation target sites on the pre-rRNA. These snoRNAs then guide the acetylation of the 18S rRNA precursor sequence by the Kre33 acetyltransferase domain (Sharma et al., 2015, 2017).

\subsubsection{The roles of RNA helicases as structural remodellers on pre- ribosomes}

For some RNA helicases, roles have been proposed in remodelling pre-ribosomal particles in ways that enable pre-rRNA processing or recruitment/release of other proteins. Such is the case of the helicase Prp43, which is proposed to promote final processing of the 18S rRNA precursor by remodelling late pre-40S particles and enabling access of the endonuclease Nob1 to its target site (Bohnsack et al., 2009; Pertschy et al., 2009). Similarly, the DEAD-box helicase Dbp3 facilitates $A_{3}$ cleavage by the RNase MRP. This helicase is proposed to disrupt secondary structures adjacent to the cleavage site, allowing the recruitment and function of the MRP complex (Kressler et al., 1999; Weaver et al., 1997). Furthermore, a recent study implicates the DEAD-box helicase Spb4 in structural rearrangements that enable incorporation of the export factor Arx1 into late pre-60S particles. The same study suggests an early role for Mak5 in remodelling the pre-rRNA to enable recruitment of the RP Rpl10 to late LSU precursors (Brüning et al., 2018). Intriguingly, Rok1 has also been proposed to promote the dissociation of Rrp5 from the SSU pre-ribosome, leading to the indirect release of snR30 (Khoshnevis et al., 2016). Besides its role in U14 snoRNA release, the RNA helicase Has1 is required for the 
remodelling of domain I of the 25S rRNA precursor, enabling incorporation of ribosomal proteins (Brüning et al., 2018; Dembowski et al., 2013). The DEAD-box helicase Dbp10 also promotes $27 \mathrm{SB}$ pre-rRNA processing, and it has been suggested to unwind a helix within the 25S rRNA precursor to allow binding of additional AFs (Manikas et al., 2016). The Ski2-like helicase Mtr4, the only helicase in the pathway that does not belong to the DEAD-box or DEAH-box families, is recruited to $5.8 \mathrm{~S}$ precursors by Nop53, and to 5 ' ETS fragments by Utp18, where it unwinds rRNA secondary structures to enable them to be degraded or trimmed by the nuclear exosome (Falk et al., 2017; Thoms et al., 2015). Although no direct RNA substrates have been suggested yet, the Dbp6 RNA helicase together with $\mathrm{Dbp} 7$ and Dbp9 have been proposed to contribute in a cooperative manner to the incorporation of the ribosomal protein Rpl3 into pre-60S particles (Rosado et al., 2007). While some RNA helicases have defined roles in ribosome biogenesis, such as Prp43 in snoRNA release from pre-ribosomal particles (Bohnsack et al., 2009), the molecular function of other RNA helicases (e.g. Dhr2, Drs1 and Fal1) remains unknown. 


\subsection{Aims and Objectives}

Ribosome biogenesis is a very dynamic and energy demanding process that enables the production of mature SSU and LSU. This pathway involves four rRNAs, 80 ribosomal proteins and over 200 trans-acting assembly factors. Among these assembly factors, RNA helicases are RNA-dependent ATPases involved in the release or association of snoRNAs, remodelling of rRNA structures and in facilitating conformational transitions along the maturation process. While several RNA helicases have ascribed roles in the process, in other cases the exact molecular function(s) and targets of RNA helicases implicated in this pathway remain unknown.

One such helicase is Fal1, a largely uncharacterised essential protein required for $40 \mathrm{~S}$ maturation (Kressler et al., 1997). Fal1 is an elF4A-like helicase, and helicases of this subfamily have been shown to be regulated by cofactors containing MIF4G domains. Interestingly, Sgd1 is an MIF4G domain-containing protein that has also been suggested to function in 405 biogenesis, raising the possibility that these proteins act together as a helicase-cofactor pair.

In this context, the objective of this work was to broaden the understanding of the roles of RNA helicases and assembly factors involved in SSU maturation by conducting functional analyses of the RNA helicase Fal1 and its putative interaction partner Sgd1.

Therefore, this study aimed to:

- Characterise the pre-rRNA processing defects arising upon Fal1 and Sgd1 depletion

- Characterise Fal1 in vitro and in vivo making use of catalytically inactive mutants

- Analyse the role of Sgd1 as a putative MIF4G domain-containing cofactor of Fal1

- Identify binding sites of Fal1/Sgd1 on pre-ribosomes

- Analyse the interactions of Sgd1 with Fal1 and RNA 


\section{Materials and Methods}

\subsection{Chemicals}

Chemicals used in this study were purchased from Sigma Aldrich, Carl Roth, Roche, GE HealthCare. Restriction enzymes were purchased from ThermoFisher Scientific. DNA and RNA oligos were synthesised by Sigma-Aldrich and IDT Integrated DNA Technologies, respectively. ${ }^{32} \mathrm{P}-\gamma$-ATP was purchased from PerkinElmer.

\subsection{Molecular Cloning}

The generation of protein expression plasmids and tagging of endogenous proteins involved well-established molecular cloning techniques such as amplification by PCR, restriction enzyme digestion and $E$. coli transformation, and were generally performed according to standard methods (Sambrook and W Russell, 2001).

\subsubsection{DNA amplification by PCR and detection of product}

Gene and coding sequences for cloning into expression vectors were amplified by Polymerase Chain Reaction (PCR) from yeast genomic DNA using specific oligonucleotides including enzymatic restriction sites. An Sgd1 coding sequence optimised for expression in E. coli was synthesised by Eurofins Genomics (Ebersberg, Germany) and used as a template for amplification of full length and Sgd1-derived fragments.

For PCR amplification, specific oligonucleotides were designed (Table 2.1) with either restriction sites for enzymes (Section 2.2.2) or complementary sequences for the Gibson Assembly Method (Section 2.2.3; Gibson, 2011).

All PCR reactions were performed following a standard protocol and specific annealing temperature for each oligonucleotide pair, as described in Table 2.2. Afterwards, PCR products were pooled together and purified using a NucleoSpin Gel and PCR Clean-up kit (Machery-Nagel) according to the manufacturer's protocol. 
Table 2.1 Oligonucleotides used for molecular cloning and mutagenesis.

\begin{tabular}{|c|c|}
\hline Name & Sequence ( $\left.5^{\prime}-3^{\prime}\right)$ \\
\hline Sgd1(CO)_Ncol_Fw & TATATATACCATGGGTCAGAAAACGGACGG \\
\hline Sgd1(CO)_Xmal_Rv & TATTATATATCCCGGGACGAGTCAATGTTTTCC \\
\hline MIF4G_Ncol_Fw & $\begin{array}{l}\text { TATATATACCATGGCTTTGTCTGAAATTAGCAAGAAGGTCAAC } \\
\text { TCCTC }\end{array}$ \\
\hline MIF4G_Xmal_Rv & $\begin{array}{l}\text { ATATATCCCGGGTTACCTGTTGTTTTTCAAGTCAGACATGGTA } \\
\text { TC }\end{array}$ \\
\hline MA3(CO)_Ncol_Fw & AGCGTATGACCATGGACATTCGCCGTGCGATTTTCATCAGC \\
\hline MIF4G(CO)_Xmal_Rv & ATACTCGCCCGGGAACGGTTATTCTTCAGGTCGGACATGG \\
\hline MIF4G(CO)_Ncol_Fw & GACAGCGCCATGGATTCAACGCTGAGTGAAATTTCG \\
\hline MA3(CO)_Xmal_Rv & AACATGTTCCCGGGCATCAAGCTTCAGAATGTCATTCGC \\
\hline MIF4G_GA_Fw & $\begin{array}{l}\text { CCATCACCACCATGCCATGGGTACCCGGGGATCCCCCGGGT } \\
\text { CAACGCTGAGTGAAATTTCGAAAAAAGTG }\end{array}$ \\
\hline MA3_GA_Rv & $\begin{array}{l}\text { GCGTGAATGTAAGCGTGACATAACTAATTACATGACTCGAGT } \\
\text { TAATCAAGCTTCAGAATGTCATTCGCCAGC }\end{array}$ \\
\hline Sgd1_GA_Rv & $\begin{array}{l}\text { GCGTGAATGTAAGCGTGACATAACTAATTACATGACTCGAGT } \\
\text { TACGAGTCAATGTTTTCCAGTTCTTTATCG }\end{array}$ \\
\hline MIF4G(CO)_Xhol_Rv & TATATATCTCGAGTTAACGGTTATTCTTCAGGTCGGACATGG \\
\hline Fal1_DQAD_Fw & $\begin{array}{l}\text { GTTCAAATGTTAGTTTTAGATCAGGCTGATGAATTATTGAGTG } \\
\text { AGACAC }\end{array}$ \\
\hline Fal1_DQAD_Rv & $\begin{array}{l}\text { GTGTCTCACTCAATAATTCATCAGCCTGATCTAAAACTAACAT } \\
\text { TTGAAC }\end{array}$ \\
\hline
\end{tabular}

In order to visualise the amplified products, a $5 \mu \mathrm{L}$ sample of the PCR reaction was mixed with $1 \mu \mathrm{L} 6 \mathrm{x}$ DNA loading dye $(0.2 \%$ bromophenol blue, $0.2 \%$ xylene cyanol, $60 \%$ glycerol, $60 \mathrm{mM}$ EDTA) and loaded onto a $1.5 \%$ agarose gel containing SafeView (ABM, $3 \mu \mathrm{L}$ in $30 \mathrm{~mL}$ gel-mix) followed by electrophoresis at $110 \mathrm{~V}$ for $60 \mathrm{~min}$ in $1 \times$ TAE buffer (40 mM Tris/HCl pH 8, $20 \mathrm{mM}$ acetic acid, $1 \mathrm{mM}$ EDTA). DNA bands were subsequently visualised with UV light. 
Table 2.2 Standard PCR reaction mix and cycle conditions.

\begin{tabular}{|c|c|c|c|c|}
\hline Component & Final Concentration & Temperature & Time & Cycles \\
\hline 10 x PfuT Buffer & $1 x$ & $95^{\circ} \mathrm{C}$ & $5 \mathrm{~min}$ & $x 1$ \\
\hline $2.5 \mathrm{mM}$ dNTPs & $200 \mu \mathrm{M}$ & $95^{\circ} \mathrm{C}$ & $30 \mathrm{sec}$ & \multirow{3}{*}{$\times 35$} \\
\hline \multirow{2}{*}{$\begin{array}{l}\text { Forward (Fw) } \\
\text { Oligonucleotide }\end{array}$} & \multirow[t]{2}{*}{$0.2 \mu \mathrm{M}$} & $55-60^{\circ} \mathrm{C}$ & $30 \mathrm{sec}$ & \\
\hline & & $72^{\circ} \mathrm{C}$ & $1 \mathrm{~min} / \mathrm{kb}$ & \\
\hline $\begin{array}{l}\text { Reverse (Rv) } \\
\text { Oligonucleotide }\end{array}$ & $0.2 \mu \mathrm{M}$ & $72^{\circ} \mathrm{C}$ & $10 \min$ & $x 1$ \\
\hline Template DNA & $80-100 \mathrm{ng}$ & $4^{\circ} \mathrm{C}$ & - & - \\
\hline PfuT polymerase & $2 U$ & & & \\
\hline $\mathrm{MQ}-\mathrm{H}_{2} \mathrm{O}$ & To $50 \mu \mathrm{L}$ & & & \\
\hline
\end{tabular}

\subsubsection{Restriction digest and ligation}

PCR products (insert DNA) were digested according to Table 2.1 and cloned into recipient plasmid DNA vectors. Digests were carried out in a total volume of $20 \mu \mathrm{L}$; insert DNA was digested using $10 \mathrm{U}$ of restriction enzyme(s) in $1 \mathrm{x}$ enzyme buffer following the manufacturer's instructions, for $2 \mathrm{~h}$ at $37^{\circ} \mathrm{C}$. The enzymatic digestion of $2 \mu \mathrm{g}$ plasmid DNA was performed under the same conditions, followed by an additional treatment with $1 \mathrm{U}$ of Alkaline phosphatase (ThermoFisher Scientific) at $37^{\circ} \mathrm{C}$ for $20 \mathrm{~min}$ to dephosphorylate the DNA.

The products of the restriction digests were separated by electrophoresis and the DNA fragments visualised as described in Section 2.2.1. DNA fragments of the expected length were excised from the gel and purified using a NucleoSpin Gel and PCR Clean-Up kit (Machery-Nagel) according to the manufacturer's protocol.

For ligation reactions, $50 \mathrm{ng}$ of plasmid DNA were ligated to insert DNA in a 1:5 molar ratio, using $5 \mathrm{U}$ of T4 DNA Ligase (ThermoFisher Scientific) and $1 \mathrm{x}$ ligase buffer in a final reaction volume of $20 \mu \mathrm{L}$. The reaction was incubated at room temperature (RT) for $1 \mathrm{~h}$ and then stored on ice, or at $16^{\circ} \mathrm{C}$ overnight.

\subsubsection{Gibson Assembly}

Alternatively, the Gibson Assembly method was used for cloning into expression vectors. The target vector was linearized by enzymatic digest as described above. Both vector DNA 
and PCR products were loaded onto an agarose gel, separated and purified after gel electrophoresis as previously described.

The ligation reaction was performed as described by Gibson, 2011. In brief, $5 \mu \mathrm{L}$ of a DNA mix (10-100 ng of vector and insert DNA, in equimolar amounts) were added to $15 \mu \mathrm{L}$ Reaction Mix containing $1 \times$ Isothermal Master mix (5\% PEG-8000, $100 \mathrm{mM}$ Tris/HCl $\mathrm{pH}$ 7.5, $10 \mathrm{mM} \mathrm{MgCl} 2,10 \mathrm{mM}$ DTT, $1 \mathrm{mM}$ NAD, $1 \mathrm{mM}$ dNTPs), $0.08 \mathrm{U}$ T5 exonuclease, $0.5 \mathrm{U}$ Phusion DNA Polymerase, $0.08 \mathrm{U}$ Taq DNA Ligase, and incubated at $50^{\circ} \mathrm{C}$ for $2 \mathrm{~h}$.

\subsubsection{E. coli transformation and extraction of plasmid DNA}

The products of all ligation reactions were used to transform a DH5 $\alpha$ strain of $E$. coli, which was also used for plasmid amplification. Routinely, $50 \mu \mathrm{L}$ of competent $E$. coli cells were incubated for $20 \mathrm{~min}$ on ice with a $20 \mu \mathrm{L}$ ligation reaction (or $5 \mathrm{ng}$ of plasmid DNA), followed by a heat shock treatment at $42^{\circ} \mathrm{C}$ for $1 \mathrm{~min}$. Cells were incubated on ice for $2 \mathrm{~min}$ and allowed to recover and express antibiotic resistance genes with $750 \mu \mathrm{L}$ of Luria Bertani (LB) medium at $37^{\circ} \mathrm{C}$ for $1 \mathrm{~h}$ while shaking $(750 \mathrm{rpm})$. Cells were pelleted by centrifugation at $5,000 \mathrm{~g}$ for $3 \mathrm{~min}$, resuspended in the remaining supernatant and plated onto LB agar containing $0.1 \mathrm{mg} / \mathrm{mL}$ Ampicillin (LB Amp).

After incubation for $16 \mathrm{~h}$ at $37^{\circ} \mathrm{C}$, single colonies were used to inoculate $4 \mathrm{~mL}$ pre-cultures (LB Amp), which were grown at $37^{\circ} \mathrm{C}$ shaking overnight. Cells from $1.5 \mathrm{~mL}$ of pre-culture were pelleted by centrifugation at $5,000 \mathrm{~g}$ for $1 \mathrm{~min}$ and used for plasmid extraction using a NucleoSpin MiniPrep kit (Machery-Nagel) and following the manufacturer's instructions.

Purified plasmids carrying the insert DNA (determined by test restriction digest, see section 2.2.2) were sent for sequencing (Eurofins Genomics) using oligonucleotides flanking the multiple cloning site (MCS) of the plasmid, and internal sequencing primers for big coding sequences.

\subsubsection{Site-directed mutagenesis}

In order to generate constructs for expression of proteins with amino acid substitutions in conserved sequence motifs, plasmids containing the FAL1 coding sequence were subjected to site-directed mutagenesis. Single-point mutations were introduced using specific oligonucleotides annealing to the target region and carrying the mutated bases (see Table 2.1). The mutagenic PCR reaction was carried out in triplicate according to the conditions detailed in table 2.3 . 
Table 2.3 Standard reaction mix and PCR conditions for site-directed mutagenesis.

\begin{tabular}{|c|c|c|c|c|}
\hline Component & Final Concentration & Temperature & Time & Cycles \\
\hline $10 \times$ PfuT Buffer & $1 x$ & $95^{\circ} \mathrm{C}$ & $30 \mathrm{sec}$ & $\mathrm{x} 1$ \\
\hline $2.5 \mathrm{mM}$ dNTPs & $200 \mu \mathrm{M}$ & $95^{\circ} \mathrm{C}$ & $30 \mathrm{sec}$ & \multirow{3}{*}{$x 18$} \\
\hline Fw Oligonucleotide & $0.25 \mu \mathrm{M}$ & $55^{\circ} \mathrm{C}$ & $60 \mathrm{sec}$ & \\
\hline Rv Oligonucleotide & $0.25 \mu \mathrm{M}$ & \multirow[t]{2}{*}{$68^{\circ} \mathrm{C}$} & $\begin{array}{l}1 \mathrm{~min} / \mathrm{kb} \\
\text { plasmid length }\end{array}$ & \\
\hline $\begin{array}{l}\text { Plasmid DNA } \\
\text { template }\end{array}$ & $50 \mathrm{ng}$ & & & \\
\hline PfuT polymerase & $2 U$ & & & \\
\hline $\mathrm{MQ}-\mathrm{H}_{2} \mathrm{O}$ & To $50 \mu \mathrm{L}$ & & & \\
\hline
\end{tabular}

After the PCR reaction, samples were placed on ice for 2 min and digested with $1 \mu \mathrm{L} D p n \mathrm{l}$ at $37^{\circ} \mathrm{C}$ for $2 \mathrm{~h}$. Reactions were then pooled together, mixed with $7.5 \mu \mathrm{L} 3 \mathrm{M} \mathrm{NaOAc} p H 5.2$ and the DNA was precipitated with $450 \mu \mathrm{L} 100 \%$ ethanol overnight at $-20^{\circ} \mathrm{C}$. The DNA was centrifuged at $20,000 \mathrm{~g}$ at $4^{\circ} \mathrm{C}$ for $20 \mathrm{~min}$, the pellet was washed in $70 \%$ ethanol and resuspended in $10 \mu \mathrm{L} M Q-\mathrm{H}_{2} \mathrm{O}$. The plasmid DNA was amplified in $E$. coli and positive clones were confirmed by sequencing (see section 2.2.4).

Table 2.4 Plasmids used in this study.

\begin{tabular}{|c|c|c|c|}
\hline ID & Name & Application & Source \\
\hline pMB031 & pRS415 & $\begin{array}{l}\text { Empty vector for protein } \\
\text { expression in yeast }\end{array}$ & $\begin{array}{l}\text { New England } \\
\text { Biolabs }\end{array}$ \\
\hline pMB141 & pRS415-Met-nHTP & $\begin{array}{l}\text { Empty vector for protein } \\
\text { expression in yeast }\end{array}$ & M. Bohnsack \\
\hline pMB142 & pRS415-Met-cHTP & $\begin{array}{l}\text { Empty vector for protein } \\
\text { expression in yeast }\end{array}$ & M. Bohnsack \\
\hline pMB533 & A8 & $\begin{array}{l}\text { Empty vector for His } 10 \text {-tagging }(\mathrm{N}- \\
\text { term) and protein expression in } E \text {. } \\
\text { coli }\end{array}$ & M. Bohnsack \\
\hline pMB535 & A21 & $\begin{array}{l}\text { Empty vector for His }{ }_{10} \text {-ProteinA- } \\
\text { tagging (N-term) and protein } \\
\text { expression in } E \text {. coli }\end{array}$ & M. Bohnsack \\
\hline
\end{tabular}




\begin{tabular}{|c|c|c|c|}
\hline pMB597 & A102 & $\begin{array}{l}\text { Empty vector for MBP-TEV- } \\
\text { tagging (N-term), His 10-tagging (C- } \\
\text { term) and protein expression in } E \text {. } \\
\text { coli }\end{array}$ & M. Bohnsack \\
\hline pMB1190 & A8-Fal1 & Recombinant expression in E. coli & M. Bohnsack \\
\hline pMB1278 & pRS415-Fal1+-500bp & Protein expression in yeast cells & M. Bohnsack \\
\hline pMB1322 & $\begin{array}{l}\text { pRS415-Met-Sgd1- } \\
\text { HTP }\end{array}$ & Protein expression in yeast cells & This study \\
\hline pMB1346 & A21-MIF4G_Sgd1 & Recombinant expression in E. coli & This study \\
\hline pMB1353 & A8-Fal1_DQAD & Recombinant expression in E. coli & This study \\
\hline pMB1356 & $\begin{array}{l}\text { pRS415- } \\
\text { Fal1_DQAD+-500bp }\end{array}$ & Protein expression in yeast cells & This study \\
\hline pMB1469 & pJAC_OsTIR1-HIS & $\begin{array}{l}\text { Template for genomic insertion of } \\
\text { OsTIR1 in HIS locus }\end{array}$ & $\begin{array}{l}\text { Morawska and } \\
\text { Ulrich, } 2013\end{array}$ \\
\hline pMB1608 & $\begin{array}{l}\text { pEX-A258- } \\
\text { Sgd1_CodOpt }\end{array}$ & $\begin{array}{l}\text { Sgd1 sequence optimised for } \\
\text { expression in E. coli }\end{array}$ & $\begin{array}{l}\text { Eurofins } \\
\text { Genomics }\end{array}$ \\
\hline pMB1613 & A102-Sgd1_CodOpt & Recombinant expression in E. coli & This study \\
\hline pMB1634 & $\begin{array}{l}\text { A102-MA3- } \\
\text { Cterm_Sgd1 }\end{array}$ & Recombinant expression in E. coli & This study \\
\hline pMB1636 & $\begin{array}{l}\text { A102-Nterm- } \\
\text { MIF4G_Sgd1 }\end{array}$ & Recombinant expression in E. coli & This study \\
\hline pMB1638 & $\begin{array}{l}\text { A102-MIF4G- } \\
\text { MA3_Sgd1 }\end{array}$ & Recombinant expression in E. coli & This study \\
\hline pMB1639 & $\begin{array}{l}\text { A102-MIF4G- } \\
\text { Cterm_Sgd1 }\end{array}$ & Recombinant expression in E. coli & This study \\
\hline pMB1671 & $\begin{array}{l}\text { pRS415-Met-HTP- } \\
\text { MIF4G-MA3_Sgd1 }\end{array}$ & Protein expression in yeast cells & This study \\
\hline pMB1672 & $\begin{array}{l}\text { pRS415-Met-HTP- } \\
\text { MIF4G-Cterm_Sgd1 }\end{array}$ & Protein expression in yeast cells & This study \\
\hline pMB1677 & $\begin{array}{l}\text { pRS415-Met-HTP- } \\
\text { Nterm-MIF4G_Sgd1 }\end{array}$ & Protein expression in yeast cells & This study \\
\hline
\end{tabular}

\subsection{Yeast Cultivation}

All yeast strains used in this study are based on the parental strain BY4741 (Brachmann et al., 1998) and were grown in YPD media (2 \% Dextrose, $2 \%$ Peptone/Tryptone, $1 \%$ Yeast Extract) or synthetic glucose media (SD, $1.9 \mathrm{~g} / \mathrm{L}$ yeast nitrogen base without amino 
acids, $5 \mathrm{~g} / \mathrm{L}$ ammonium sulphate, $2 \%$ dextrose, $0.79 \mathrm{~g} / \mathrm{L}$ complete supplemented mixture drop out: complete).

For selective growth, yeast strains were grown in SD -His medium $(1.9 \mathrm{~g} / \mathrm{L}$ yeast nitrogen base without amino acids, $5 \mathrm{~g} / \mathrm{L}$ ammonium sulphate, $2 \%$ dextrose, $0.77 \mathrm{~g} / \mathrm{L}$ complete supplemented mixture drop out: -His), SD -Leu medium (1.9 g/L Yeast Nitrogen Base without amino acids, $5 \mathrm{~g} / \mathrm{L}$ ammonium sulphate, $2 \%$ Dextrose, $0.69 \mathrm{~g} / \mathrm{L}$ complete supplemented mixture drop out: -Leu) or SD-Ura medium (1.9 g/L Yeast Nitrogen Base without amino acids, $5 \mathrm{~g} / \mathrm{L}$ ammonium sulphate, $2 \%$ Dextrose, $0.77 \mathrm{~g} / \mathrm{L}$ complete supplemented mixture drop out: -Ura).

Routinely, strains were stored at $-80^{\circ} \mathrm{C}$ in glycerol stocks (medium containing $15 \%$ glycerol) streaked out on appropriate plates and grown at $30^{\circ} \mathrm{C}$ for 2 days. Single colonies were used to inoculate $4 \mathrm{~mL}$ cultures, which were grown at $30^{\circ} \mathrm{C}$ while shaking for $16 \mathrm{~h}$. The stationary phase cultures were then used either as such or diluted into larger volumes for generating exponential phase cultures.

\subsection{Yeast transformation}

For the generation of yeast strains, tagging cassettes were amplified by PCR according to standard protocol (section 2.2.1) using as template pYM14 (Janke et al., 2004) for 6xHAtagging or pHyg-AID*-6xHA (Morawska and Ulrich, 2013) for auxin-degron-tagging (Table 2.4). Oligonucleotides were designed for amplification of the tagging cassettes, and target specific sequences were added to allow for homologous recombination of the PCR product with genomic DNA (Table 2.5).

Table 2.5 Oligonucleotides used for amplification of tagging cassettes.

$\begin{array}{ll}\text { Name } & \text { Sequence (5'-3') } \\ \text { Fal1_Janke_S3 } & \begin{array}{l}\text { GAAAAATTCTATTCTATCAAAATAAATCCGATGCCAGCAAATTTTGCA } \\ \text { GAATTATCACGTACGCTGCAGGTCGAC }\end{array} \\ \text { Fal1_Janke_S2 } & \begin{array}{l}\text { GCCGCATGGATGAGACTCTCTTAACTTGTTTATGTAGGTATATGTTCT } \\ \text { CTATATTTTAATCGATGAATTCGAGCTCG }\end{array} \\ \text { Fal1_Aux_Fw } & \begin{array}{l}\text { CTATCAAAATAAATCCGATGCCAGCAAATTTTGCAGAATTATCACCTA } \\ \text { AAGATCCAGCCAAAC }\end{array} \\ \text { Fal1_Aux_Rv } & \begin{array}{l}\text { GGATGAGACTCTCTTAACTTGTTTATGTAGGTATATGTTCTCTATATTC } \\ \text { TGATATCATCGATGAATTC }\end{array}\end{array}$




\section{Sgd1_Aux_Fw CAAAAGCTGCCAAATCTATTATAGACAAAGAACTGGAAAATATAGATC CTAAAGATCCAGCCAAAC \\ Sgd1_Aux_Rv GGTTATAGTTGAGCAAATAAAGAATACAAAATTTATTCCAAAAAACAC TGATATCATCGATGAATTC}

To allow exogenous expression of proteins or to tag endogenous proteins, yeast cells were transformed with exogenous DNA from plasmids or PCR products, respectively. Additionally, a plasmid carrying the OsTIR1 gene was used for homologous recombination with genomic DNA after enzymatic digestion with Pmel (as described in section 2.2.2).

For transformation of yeast cells, $1 \mathrm{~mL}$ stationary phase culture of the parental strain was used for inoculating $50 \mathrm{~mL}$ of YPD medium and incubated at $30^{\circ} \mathrm{C}$ shaking until $\mathrm{OD}_{600}=0.7$ was reached. Cells were harvested by centrifugation at $4,000 \mathrm{~g}$ for $5 \mathrm{~min}$ and washed once with $10 \mathrm{~mL}$ sterile water. The cell pellet was resuspended in $1 \mathrm{~mL}$ sterile water, transferred to a $1.5 \mathrm{~mL}$ tube and centrifuged at 5,000 rpm for $5 \mathrm{~min}$. Cells were then washed once with $1.5 \mathrm{~mL} 1 \times \mathrm{TE} / \mathrm{LiAc}$ buffer $(10 \mathrm{mM}$ Tris/HCl pH 7.5, $1 \mathrm{mM}$ EDTA, $100 \mathrm{mM}$ lithium acetate $\mathrm{pH} 7.5$ ) and resuspended in $200 \mu \mathrm{L} 1 \times \mathrm{TE} / \mathrm{LiAc}$ buffer (to a concentration of approx. $2 \times 10^{9}$ cells $/ \mathrm{mL}$ ). Per transformation reaction, $50 \mu \mathrm{L}$ cell suspension were mixed with $5 \mu \mathrm{L}$ denatured salmon sperm DNA (10 mg/ml, preheated for $20 \mathrm{~min}$ at $95^{\circ} \mathrm{C}$ and cooled down on ice) and the DNA of interest (12 $\mu \mathrm{L}$ PCR product or $0.5 \mu \mathrm{g}$ plasmid DNA). Immediately, $300 \mu \mathrm{L}$ PEG/TE/LiAc buffer (40\% polyethylene glycol 4000, $10 \mathrm{mM}$ Tris/HCl pH 7.5, $1 \mathrm{mM}$ EDTA, $10 \mathrm{mM}$ lithium acetate $\mathrm{pH}$ 7.5) were mixed into the reaction. Cells were incubated $30 \mathrm{~min}$ at $30^{\circ} \mathrm{C}$ while shaking (750 rpm) and then subjected to a heat shock at $42^{\circ} \mathrm{C}$ for $15 \mathrm{~min}$. Finally, $800 \mu \mathrm{L}$ sterile water were added to the transformation reaction, cells were pelleted by centrifugation at $13,000 \mathrm{rpm}$ for $10 \mathrm{sec}$ and resuspended in $100 \mu \mathrm{L}$ sterile water.

In the case of auxotrophic selection of transformed cells, the cell suspension was plated onto the appropriate drop out media and incubated at $30^{\circ} \mathrm{C}$ for 2 days. In the case of selection by antibiotics, the resuspended cell pellet was plated onto YPD plates, incubated overnight at $30^{\circ} \mathrm{C}$ and then replica plated onto selective media. For both selection methods, after growth at $30^{\circ} \mathrm{C}$ for 2 days, single colonies were streaked out and further selected twice to avoid mixed colonies from different genetic backgrounds.

Tagging of endogenous proteins was checked by western blotting (see section 2.8) and positive strains were stored at $-80^{\circ} \mathrm{C}$ in glycerol stocks. All yeast strains used during this study are detailed in table 2.6. 
Table 2.6 Yeast strains used in this study.

\begin{tabular}{|c|c|c|c|}
\hline Strain ID & Name & Genotype & Source \\
\hline YMB006 & BY4741a & $\begin{array}{l}\text { MATa; his } 3 \Delta 1 \text {; leu } 2 \Delta 0 \\
\text { met15 } \Delta 0 ; \text {; ura3 } \Delta 0\end{array}$ & $\begin{array}{l}\text { Brachmann et al., } \\
1998\end{array}$ \\
\hline YMB146 & pTetO $7-$ Rok1 & $\begin{array}{l}\text { YMB279; pTtetO } 7-3 \times \text { HA-Rok1 } \\
\text { (NatNT2) }\end{array}$ & Bohnsack lab \\
\hline YMB279 & pTetO7 parent & $\begin{array}{l}\text { YMB006; tTA::LYS2; } \\
\text { tetR'::URA3 K.I.::LEU2 }\end{array}$ & $\begin{array}{l}\text { (Alexander et al., } \\
\text { 2010) }\end{array}$ \\
\hline YMB1175 & Fal1-HA & YMB006; Fal1-3xHA (hpnNT) & Bohnsack lab \\
\hline YMB1461 & OsTIR1 & $\begin{array}{l}\text { YMB006; pTdh3- } \\
\text { OsTIR1::HIS3MX6 }\end{array}$ & This study \\
\hline YMB1584 & $\begin{array}{l}\text { OsTIR1 Fal1-HA- } \\
\text { AID }\end{array}$ & $\begin{array}{l}\text { YMB1461; Fal1-6xHA-AID } \\
\text { (hpnNT) }\end{array}$ & This study \\
\hline YMB1629 & $\begin{array}{l}\text { OsTIR1 Sgd1- } \\
\text { HA-AID }\end{array}$ & $\begin{array}{l}\text { YMB1461; Sgd1-6xHA-AID } \\
\text { (hpnNT) }\end{array}$ & This study \\
\hline YMB1632 & $\begin{array}{l}\text { OsTIR1 Fal1-HA- } \\
\text { AID + EV }\end{array}$ & YMB1584; pMB031::LEU2 & This study \\
\hline YMB1634 & $\begin{array}{l}\text { OsTIR1 Fal1-HA- } \\
\text { AID + Fal1-WT }\end{array}$ & YMB1584; pMB1278::LEU2 & This study \\
\hline YMB1656 & OsTIR1 Fal1-HA & YMB1461; Fal1-6xHA & This study \\
\hline YMB1672 & $\begin{array}{l}\text { OsTIR1 Fal1-HA- } \\
\text { AID + Fal1- } \\
\text { DQAD }\end{array}$ & YMB1584; pMB1356::LEU2 & This study \\
\hline YMB1684 & Sgd1-HTP & YMB006; Sgd1-HTP:: URA3 & This study \\
\hline YMB1852 & $\begin{array}{l}\text { Sgd1_MIF4G- } \\
\text { MA3 }\end{array}$ & YMB1629; pMB1671::LEU2 & This study \\
\hline YMB1853 & $\begin{array}{l}\text { Sgd1_MIF4G- } \\
\text { Cterm }\end{array}$ & YMB1629; pMB1672::LEU2 & This study \\
\hline YMB1886 & $\begin{array}{l}\text { Sgd1-Nterm- } \\
\text { MIF4G }\end{array}$ & YMB1629; pMB1677::LEU2 & This study \\
\hline
\end{tabular}

\subsection{Depletion of endogenous proteins from yeast using the auxin-inducible degron system}

As FAL1 and SGD1 are both essential genes and their deletion leads to non-viable cells, endogenous proteins were transiently depleted using the auxin-inducible degron system, as described by Morawska and Ulrich, 2013. In yeast strains expressing both the OsTIR1 
complex and the protein of interest degron-tagged (AID-tag), addition of auxin to the media rapidly targets the protein for degradation by the proteasome. As this approach involves C-terminal-tagging of the proteins, expression in the absence auxin is from the endogenous promoter and therefore at an endogenous expression level.

In order to avoid secondary effects arising from protein depletion, an optimal depletion time was determined. For this, yeast cultures were grown at $30^{\circ} \mathrm{C}$ in $\mathrm{SD}$ media until $\mathrm{OD}_{600}=0.6$ was reached. To start the depletion, IAA (3-indoleacetic acid, Sigma-Aldrich), resuspended in $100 \%$ ethanol, was added to the cultures to a final concentration of $0.5 \mathrm{mM}$. The same volume of $100 \%$ ethanol was added to control samples. Cultures were further grown and samples were taken every $30 \mathrm{~min}$ corresponding to $\mathrm{OD}_{600}=4$. Protein levels were determined after TCA precipitation of proteins and immunoblotting. The time of depletion was determined as the earliest time point when the protein was no longer detectable by immunoblotting using an antibody against the HA-epitope of the C-terminal AID-tag.

\subsection{In vivo Complementation Assay}

To analyse the effects of specific mutations on protein activity, in vivo complementation assays were performed. For this, yeast strains for the depletion of endogenous proteins were transformed with expression plasmids encoding a wild-type or a mutant version of the protein, and additionally, a strain was transformed with the empty vector to use as a control (see section 2.4 and Table 2.6).

Yeast cultures were grown to mid-log phase at $30^{\circ} \mathrm{C}$ in SD -Leu medium and submitted to depletion for 30 and $60 \mathrm{~min}$ as described in section 2.5. Cells were harvested by centrifugation at $4,500 \mathrm{~g}$ at room temperature (RT) for $5 \mathrm{~min}$, and the cell pellets were washed once with $1 \times$ PBS buffer $\left(137 \mathrm{mM} \mathrm{NaCl}, 2.7 \mathrm{mM} \mathrm{KCl}, 10 \mathrm{mM} \mathrm{Na}_{2} \mathrm{HPO}_{4}, 1.8 \mathrm{mM}\right.$ $\mathrm{KH}_{2} \mathrm{PO}_{4}$ ). After centrifugation at $4,500 \mathrm{~g}$ for $5 \mathrm{~min}$ at $\mathrm{RT}$, the supernatants were discarded and cell pellets were frozen in liquid nitrogen, stored at $-80^{\circ} \mathrm{C}$ and later used for RNA extraction (see section 2.9).

\subsection{Enrichment of endogenous complexes on IgG sepharose}

Endogenous protein-RNA complexes were purified from yeast lysates via His-TEV protease cleavage site-ProteinA (HTP)-tagged proteins. A yeast strain expressing an HTPtagged protein of interest was grown in $1 \mathrm{~L} \mathrm{SD}$ medium at $30^{\circ} \mathrm{C}$ to $\mathrm{OD}_{600}=0.8$ and the cells were harvested by centrifugation at $6,000 \mathrm{~g}$ for $5 \mathrm{~min}$ at $4^{\circ} \mathrm{C}$. The cell pellet was resuspended in $20 \mathrm{~mL} 1 \times$ PBS buffer and transferred to a $50 \mathrm{~mL}$ falcon tube. After 
centrifugation at $4,500 \mathrm{~g}$ for $5 \mathrm{~min}$ at $4^{\circ} \mathrm{C}$, the cells were resuspended in 1 pellet volume of TMN150 buffer (50 mM Tris/HCl pH 7.8, $150 \mathrm{mM} \mathrm{NaCl}, 1.5 \mathrm{mM} \mathrm{MgCl}_{2}, 0.1 \% \mathrm{NP}-40$, $5 \mathrm{mM} \beta$-mercaptoethanol) containing protease inhibitor (+ PI, 1 tablet cOmplete Mini protease inhibitor mix in $25 \mathrm{~mL}$ buffer, Roche). Cells were ground in liquid nitrogen three times using a mortar and pestle, and the lysate was stored on ice to thaw, followed by centrifuging at $20,000 \mathrm{~g}$ at $4^{\circ} \mathrm{C}$ for $30 \mathrm{~min}$. $100 \mu \mathrm{L}$ slurry lgG sepharose beads (GE Healthcare) were washed three times with $1 \mathrm{~mL}$ TMN150 buffer by centrifuging (500 rpm, $4^{\circ} \mathrm{C}, 1 \mathrm{~min}$ ) and incubated with cleared lysate at $4^{\circ} \mathrm{C}$ for $2 \mathrm{~h}$ while rotating. The beads were then washed three times with $1 \mathrm{~mL}$ TMN150 buffer and centrifuging at $500 \mathrm{~g}$ for $1 \min$ at $4^{\circ} \mathrm{C}$.

The purified complexes were further analysed either by RNA extraction from the beads (see section 2.9) or by TCA precipitation of proteins (see section 2.8). Before TCA precipitation, the complexes were eluted from the IgG beads by TEV protease cleavage. After the last washing step, the beads were resuspended in $200 \mu \mathrm{L}$ TMN150 buffer and transferred to a Mobicol column (bioRad, $35 \mathrm{~nm}$ pore size filter). $1.6 \mu \mathrm{g}$ GST-TEV (Glutathione-S-transferase - Tobacco Etch virus) were added to the suspension and incubated at $4^{\circ} \mathrm{C}$ overnight. The purified complexes were collected in a fresh tube after centrifugation at $500 \mathrm{~g}$ for $1 \mathrm{~min}$.

\subsection{Total protein precipitation and SDS-PAGE}

Proteins were precipitated from total yeast lysates in order to check newly generated yeast strains or to compare protein levels under different growth conditions. Denatured proteins were separated by size using denaturing sodium dodecyl sulphate polyacrylamide gel electrophoresis (SDS-PAGE). Following electrophoresis, proteins were visualised by Coomassie staining, or transferred to a nitrocellulose membrane for detection with specific antibodies (section 2.9).

To obtain a total yeast lysate, cell pellets were resuspended in $150 \mu \mathrm{L} M Q-\mathrm{H}_{2} \mathrm{O}, 150 \mu \mathrm{L}$ glass beads were added and cells were disrupted by vortexing at max. speed for $5 \mathrm{~min}$ at $4^{\circ} \mathrm{C}$. Proteins were then precipitated by addition of $1 \mathrm{~mL} 15 \%$ trichloroacetic acid (TCA, Sigma-Aldrich) and incubation on ice for $10 \mathrm{~min}$. The supernatant was transferred into a fresh tube and centrifuged at $20,000 \mathrm{~g}$ for $20 \mathrm{~min}$ at $4^{\circ} \mathrm{C}$. The precipitated protein pellet was washed twice with $1 \mathrm{~mL}$ ice-cold acetone and centrifuged at 20,000 g for $20 \mathrm{~min}$ at $4^{\circ} \mathrm{C}$. The supernatant was discarded, and the protein pellet air-dried and resuspended in $32 \mu \mathrm{L} 1 \times$ SDS buffer $(60 \mathrm{mM}$ Tris/ $\mathrm{HCl}$ pH 6.8, $2 \%$ SDS, $0.01 \%$ bromophenol blue, $1.25 \% \beta$-mercaptoethanol). 
Before loading onto a denaturing polyacrylamide gel, protein samples were incubated at $95^{\circ} \mathrm{C}$ for $15 \mathrm{~min}$. SDS-PAGE gels contained a $5 \%$ polyacrylamide (PAA) stacking gel and a resolving gel of $8 \%$ or $10 \%$ PAA concentration, for resolution of larger (>100 kDa) or smaller (approx.. $60 \mathrm{kDa}$ ) proteins, respectively. Electrophoresis was done in $1 \mathrm{x}$ Lämmli Buffer (25 mM Tris, $192 \mathrm{mM}$ glycine, $0.1 \%$ SDS) at $30 \mathrm{~mA}$ for $1 \mathrm{~h}$.

\subsection{Western blotting and Coomassie staining}

For detection by specific antibodies, proteins were transferred from a polyacrylamide gel to a nitrocellulose membrane (GE Healthcare) by wet electroblotting at $110 \mathrm{~V}$ for $60 \mathrm{~min}$ at $4^{\circ} \mathrm{C}$ in $1 \times$ Western Blot buffer (25 mM Tris, $192 \mathrm{mM}$ glycine, $0.05 \%$ SDS, 20 \% methanol). Non-specific binding of the antibodies to the membrane was prevented by incubation with blocking solution ( $5 \%$ milk, $1 \%$ Tween2000, in 1 x PBS buffer) for $1 \mathrm{~h}$ at RT. Afterwards, the membrane was incubated overnight at $4^{\circ} \mathrm{C}$ with an appropriate dilution of primary antibody in blocking solution (see table 2.7).

Table 2.7 Antibodies used in this study.

\begin{tabular}{lll}
\hline Name & Dilution & Provider \\
\hline$\alpha-H A$ & $1: 500$ & Sigma-Aldrich Chemie GmbH \\
$\alpha-P g k 1$ & $1: 7,500$ & Life Technologies GmbH \\
$\alpha-$ MBP & $1: 10,000$ & New England BioLabs \\
$\alpha$-His & $1: 3,000$ & (Sigma-Aldrich Chemie GmbH \\
$\alpha$-mouse (800) & $1: 10,000$ & Li-COR \\
$\alpha$-mouse (680) & $1: 10,000$ & Li-COR \\
Goat-anti-mouse & $1: 10,000$ & Jackson ImmunoResearch Europe Ltd. \\
\hline
\end{tabular}

After incubation with the primary antibody, the membrane was washed $3 x$ with $1 \times$ PBS-T buffer (1 x PBS buffer, $1 \%$ Tween2000) and incubated with the secondary antibody (diluted in blocking solution) at RT for $1 \mathrm{~h}$. Secondary antibodies conjugated with horseradish peroxidase (HRP) were used for Enhanced ChemoLumminiscence (ECL) detection, and IRDye conjugated secondary antibodies were used for fluorescent detection. 
For visualisation of recombinantly expressed and purified proteins, proteins separated by SDS-PAGE were stained with Coomassie staining solution $(1 \mathrm{mg} / \mathrm{mL}$ Coomassie, $45 \%$ ethanol, $10 \%$ acetic acid) at RT for $1 \mathrm{~h}$ while shaking, and washed with destaining solution (30\% ethanol, $10 \%$ acetic acid) overnight.

\subsection{RNA extraction from yeast}

Total RNA was extracted from yeast cells or from purified complexes, and later used for analysis of pre-rRNA processing or protein associated RNAs.

In preparation for total RNA extraction, yeast cell pellets were washed once with $10 \mathrm{~mL}$ PBS buffer and centrifuged at $5,000 \mathrm{~g}$ for $5 \mathrm{~min}$ at $4^{\circ} \mathrm{C}$. Cells were resuspended in $200 \mu \mathrm{L}$ GTC Mix (2 M guanidine thiocyanate, $25 \mathrm{mM}$ Tris/HCl pH 8.0, $5 \mathrm{mM}$ EDTA pH 8.0, 1 \% Nlauroylsarcosine, $150 \mathrm{mM} \beta$-mercaptoethanol) and transferred into a new $15 \mathrm{~mL}$ tube. $600 \mu \mathrm{L}$ glass beads and $200 \mu \mathrm{L}$ Roti-Aqua-Phenol (Carl Roth) were added to the suspension, and cells were lysed by vortexing for $5 \mathrm{~min}$ at $4^{\circ} \mathrm{C}$. For extraction of RNAs associated with complexes immobilised on IgG beads, the beads were resuspended in $3 \mathrm{~mL}$ GTC Mix and transferred to a new $15 \mathrm{~mL}$ falcon tube. For extraction from both yeast lysate and beads, an additional $3 \mathrm{~mL}$ of Roti-Aqua-Phenol were added to the suspension, followed by incubation for $5 \mathrm{~min}$ at $65^{\circ} \mathrm{C}$. Subsequently, the suspension was stored on ice and incubated for $5 \mathrm{~min}$. $1.6 \mathrm{~mL}$ sodium acetate $\mathrm{mix}(100 \mathrm{mM}$ sodium acetate $\mathrm{pH} 5.2$, $1 \mathrm{mM}$ EDTA pH 8, $10 \mathrm{mM}$ Tris $/ \mathrm{HCl} \mathrm{pH} 8$ ) and $3 \mathrm{~mL}$ chloroform were added to the icecooled suspension and the suspension was mixed by vortexing. The organic phase was separated from the aqueous phase by centrifugation at $4,500 \mathrm{rpm}$ for $30 \mathrm{~min}$ at $4^{\circ} \mathrm{C} .5 \mathrm{~mL}$ of the aqueous phase (upper phase) were transferred into a new $15 \mathrm{~mL}$ tube, for a second extraction step. $5 \mathrm{~mL} \mathrm{PCl}$ (phenol:chloroform:isoamyl alcohol in 25:24:1 ratio) were added, the suspension vortexed and centrifuged at $4,500 \mathrm{rpm}$, for $5 \mathrm{~min}$, at $4^{\circ} \mathrm{C}$. Again, the aqueous phase $(4.5 \mathrm{~mL})$ was transferred into a new $15 \mathrm{~mL}$ tube and mixed with chloroform in a $1: 1(\mathrm{v} / \mathrm{v})$ ratio. After vortexing thoroughly, phases were separated by centrifugation (4,500 rpm, $\left.5 \mathrm{~min}, 4^{\circ} \mathrm{C}\right)$ and $4 \mathrm{~mL}$ of the upper phase were transferred into a new tube. $30 \mu \mathrm{g}$ GlycoBlue (Invitrogen) were added to the aqueous phase and the RNA was precipitated by addition of $11 \mathrm{~mL}$ ethanol and storage at $-20^{\circ} \mathrm{C}$, overnight. The sample was then centrifuged at $4,500 \mathrm{rpm}$ for $30 \mathrm{~min}$ at $4^{\circ} \mathrm{C}$, the supernatant discarded and the RNA pellet was washed once with $2 \mathrm{~mL} 70 \%$ ethanol. After centrifugation (4,500 rpm, $10 \mathrm{~min}$, $4^{\circ} \mathrm{C}$ ) of the sample, the supernatant was removed, and the RNA pellet was dried and resuspended in $30 \mu \mathrm{L} M Q-\mathrm{H}_{2} \mathrm{O}$. 


\subsection{Denaturing agarose gel electrophoresis and northern blotting for pre- rRNAs}

rRNA precursors were separated by electrophoresis in 1 x BPTE buffer (10 mM Pipes, $30 \mathrm{mM}$ Bis-Tris, $1 \mathrm{mM}$ EDTA $\mathrm{pH} 8$ ) and detected by northern blotting with specific radiolabelled probes (Table 2.8). Prior to loading, $5 \mu \mathrm{g}$ total RNA were mixed with 5 volumes of glyoxal dye (20\% glyoxal, $61 \%$ DMSO, $5 \%$ glycerol, $0.1 \%$ ethidium bromide in $1 \times$ BPTE) and incubated at $55^{\circ} \mathrm{C}$ for $1 \mathrm{~h}$. Samples were then loaded onto a $1.2 \%$ agarose gel and resolved by electrophoresis in $1 \times$ BPTE buffer at $60 \mathrm{~V}$ for $16 \mathrm{~h}$. Abundant RNAs were then visualised by exposure to UV light to verify separation. The gel was washed for 20 min with $100 \mathrm{mM} \mathrm{NaOH}$, two times for 15 min with Tris-salt buffer (0.5 M Tris/ $\mathrm{HCl} \mathrm{pH} \mathrm{7.4,} 1.5 \mathrm{M} \mathrm{NaCl}$ ) and once for 15 min with $6 \times \mathrm{SSC}$ buffer $(900 \mathrm{mM}$ $\mathrm{NaCl}, 100 \mathrm{mM}$ sodium citrate, $\mathrm{pH}$ 7). The RNA was transferred onto a Hybond-N+ nylon membrane (previously equilibrated in 6 x SSC buffer) by vacuum blotting at 300 mbar bar for $1.5 \mathrm{~h}$. Finally, the RNA was crosslinked to the membrane using UV light of $0.12 \mathrm{~J} / \mathrm{cm}^{2}$ (UV Stratalinker) twice.

The membrane was stained with methylene blue solution ( $0.3 \mathrm{M}$ sodium acetate $\mathrm{pH} 5.2$, methylene blue) to visualise the most abundant RNA species, and then prehybridised with $30 \mathrm{~mL}$ SES1 buffer (0.5 M sodium phosphate $\mathrm{pH} 7.2,7 \%$ SDS, $1 \mathrm{mM}$ EDTA pH 8.0) at $37^{\circ} \mathrm{C}$ for $30 \mathrm{~min}$ while rotating. rRNA precursors were detected by hybridising specific antisense radioactively-labelled DNA oligonucleotides (Table 2.7) with the membrane. Oligonucleotide probes were labelled by mixing $2 \mu \mathrm{L} 10 \mathrm{mM}$ oligonucleotide with $2 \mu \mathrm{L}$ 10 x T4 polynucleotide kinase buffer A (ThermoFisher Scientific), 10 U T4 polynucleotide kinase (PNK) and $2 \mu \mathrm{L}{ }^{32} \mathrm{P}-\gamma$-ATP in a final reaction volume of $20 \mu \mathrm{L}$, followed by incubation at $37^{\circ} \mathrm{C}$ for $40 \mathrm{~min}$. The radiolabelled oligonucleotide was then diluted in $30 \mathrm{~mL}$ SES1 buffer and hybridised with the membrane rotating in a tube overnight at $37^{\circ} \mathrm{C}$.

Table 2.8 Oligonucleotides used as Northern blot probes.

\begin{tabular}{lll}
\hline ID & Name & Sequence $\left(\mathbf{5}^{\prime}-\mathbf{3}^{\prime}\right)$ \\
\hline 1461 & $\mathrm{~b}$ & TGTTACCTCTGGGCCC \\
1462 & 004 & CGGTTTTAATTGTCCTA \\
1468 & 020 & TGAGAAGGAAATGACGCT \\
7194 & $\mathrm{c}$ & CCAGTTACGAAAATTCTTG \\
7195 & $\mathrm{a}$ & GAAGCAACAAGCAGTAAAAAAG \\
\hline
\end{tabular}


The membrane was washed once for 30 min with 6 x SSC buffer and once for 30 min with $2 \times$ SSC buffer containing $0.1 \%$ SDS at $37^{\circ} \mathrm{C}$, while shaking. Once dried, the membrane was exposed to a phosphorimager screen. After exposure for one or two days, radioactive signals were detected using a Typhoon FLA 9500 phosphorimager (GE Healthcare).

For re-probing, the membrane was first striped with $400 \mathrm{~mL}$ Stripping buffer $(0.1 \times$ SSC, $0.1 \%$ SDS, previously heated to $100^{\circ} \mathrm{C}$ ) for $1 \mathrm{~h}$ shaking, at $70^{\circ} \mathrm{C}$. Once stripped, the membrane was pre-hybridised in SES1 buffer and re-probed as described above.

\subsection{In culturo UV-crosslinking and analysis of cDNA (CRAC)}

Specific protein-RNA interactions were analysed using the UV-crosslinking and analysis of $\underline{c}$ DNA (CRAC) method, previously described by Bohnsack et al., 2012 and Granneman et al., 2009. This technique crosslinks proteins covalently to their associated RNAs by UVirradiation, followed by purification of the protein-RNA complexes and preparation of a cDNA library that is analysed by next-generation sequencing. All CRAC experiments presented in this study were performed by Philipp Hackert.

Yeast strains expressing HTP-tagged proteins were grown in $1 \mathrm{~L} \mathrm{SD}$ or SD -Leu to midlog phase $\left(\mathrm{OD}_{600}=0.8\right)$ and, if required, depleted of endogenous proteins as previously described (see section 2.5). The cells were crosslinked with a custom built crosslinker (iTRIC: in culturo temperature regulated interaction crosslinker) by UV irradiation at $254 \mathrm{~nm}\left(1.6 \mathrm{~J} / \mathrm{cm}^{2}\right)$ for $30 \mathrm{~min}$ at a constant temperature of $\sim 22^{\circ} \mathrm{C}$. Once irradiated, cells were harvested by centrifugation $\left(4,500 \mathrm{~g}, 4^{\circ} \mathrm{C}, 5 \mathrm{~min}\right)$, resuspended in $20 \mathrm{~mL}$ PBS and transferred to a $50 \mathrm{~mL}$ falcon tube. After centrifugation at $4,500 \mathrm{~g}, 4^{\circ} \mathrm{C}$ for $5 \mathrm{~min}$, the supernatant was discarded, the cell pellet was frozen in liquid nitrogen and stored at $-80^{\circ} \mathrm{C}$.

Before cell lysis, the cell pellet was thawed on ice and resuspended in 1 volume of TMN150 + PI buffer. Cells were disrupted with $3 \mathrm{~mL}$ zirconia beads (Thistle Scientific) by vortexing $5 \times 1 \mathrm{~min}$, with $1 \mathrm{~min}$ incubation on ice in between. The cell suspension was diluted with 3 pellet volumes of TMN150 + PI buffer and centrifuged at $4,500 \mathrm{~g}$ at $4^{\circ} \mathrm{C}$ for $20 \mathrm{~min}$. The supernatant was transferred into a $1.5 \mathrm{~mL}$ tube and cleared by centrifugation at $20,000 \mathrm{~g}, 4^{\circ} \mathrm{C}$ for $20 \mathrm{~min}$. HTP-tagged proteins, and associated complexes, were purified from the cleared lysate using IgG sepharose beads as described in section 2.7. After the last washing step, beads were resuspended in $600 \mu \mathrm{L}$ TMN150 buffer. $8 \mu \mathrm{g}$ GSTTEV protease were added followed by incubation overnight at $4^{\circ} \mathrm{C}$, rotating. After TEV cleavage, the supernatant was transferred to a new tube and treated with $0.1 \mathrm{U}$ RNace-lt (Agilent Technologies) for $30 \mathrm{sec}$ at $37^{\circ} \mathrm{C}$. After incubation, the reaction was stopped in 
Stopping Reagent (6 M Guanidium/ $\mathrm{HCl}, 300 \mathrm{mM} \mathrm{NaCl}, 10 \mathrm{mM}$ Imidazole $\mathrm{pH}$ 8.0) by vortexing.

For purification of protein-RNA complexes under denaturing conditions, $100 \mu \mathrm{L}$ Ni-NTA bead slurry (Qiagen) were washed three times with $500 \mu \mathrm{L}$ Wash Buffer $1(50 \mathrm{mM}$ Tris $/ \mathrm{HCl}$ $\mathrm{pH} 7.8,300 \mathrm{mM} \mathrm{NaCl}, 10 \mathrm{mM}$ Imidazole, $6 \mathrm{M}$ Guanidine/HCl, $0.1 \% \mathrm{NP}-40,5 \mathrm{mM}$ $\beta$-mercaptoethanol), with a centrifugation step $\left(500 \mathrm{~g}, 4^{\circ} \mathrm{C}, 10 \mathrm{sec}\right)$ between each wash. The protein-RNA complexes were incubated with the equilibrated Ni-NTA beads for $2 \mathrm{~h}$ at $4^{\circ} \mathrm{C}$ while rotating. Afterwards, beads were washed twice with $750 \mu \mathrm{L}$ Wash Buffer 1 and three times with $750 \mu \mathrm{L}$ PNK buffer $(50 \mathrm{mM}$ Tris/HCl pH 7.8, $10 \mathrm{mM} \mathrm{MgCl}$, $0.5 \% \mathrm{NP}-40$, $5 \mathrm{mM} \beta$-mercaptoethanol). The beads were transferred to a Mobicol spin column and the remaining buffer was discarded after centrifugation at $500 \mathrm{~g}$ for $10 \mathrm{sec}$.

The co-precipitated RNAs were treated with alkaline phosphatase (TSAP, Promega) to remove the 5' and 3' phosphates left by RNase cleavage. The Ni-NTA beads were resuspended in $80 \mu \mathrm{L}$ TSAP-mix containing $8 \mathrm{U}$ thermo-sensitive alkaline phosphatase (TSAP, Promega) and $80 \mathrm{U}$ RNasin (ribonuclease inhibitors, Promega) in 1 x PNK buffer, and incubated for $30 \mathrm{~min}$ at $37^{\circ} \mathrm{C}$. After the treatment, the beads were washed once with $400 \mu \mathrm{L}$ Wash Buffer 1 and three times with $400 \mu \mathrm{L} 1$ x PNK buffer.

For subsequent reverse transcription, PCR amplification and sequencing steps, adaptors were ligated to the RNA fragments on the beads. First, a 3' adaptor was ligated to the RNA by resuspending the beads in $80 \mu \mathrm{L}$ of $3^{\prime}$ linker-mix, containing $60 \mathrm{U}$ RNasin, $800 \mathrm{U}$ RNA ligase II mutant (Epicentre), 1.25 M RA3 3' Linker and 10 \% PEG8000 in 1 x PNK buffer, followed by overnight incubation at $16^{\circ} \mathrm{C}$. After ligation, the beads were washed as described above ( $1 \times 400 \mu \mathrm{L}$ Wash Buffer $1,3 \times 400 \mu \mathrm{L} 1 \times$ PNK buffer; $\left.500 \mathrm{~g}, 5 \mathrm{~min}, 4^{\circ} \mathrm{C}\right)$ and the RNA 5' ends were radioactively phosphorylated to enable detection of the purified protein-RNA complexes by autoradiography. For the phosphorylation reaction, the beads were resuspended in $80 \mu \mathrm{L}$ PNK mix, containing $80 \mathrm{U}$ T4 PNK (New England Biolabs), $60 \mathrm{U}$ RNasin, $40 \mu \mathrm{Ci}{ }^{32} \mathrm{P}-\gamma-\mathrm{ATP}, 1 \times \mathrm{PNK}$ buffer, and incubated at $37^{\circ} \mathrm{C}$ for $40 \mathrm{~min}$. The reaction was then supplemented with $1 \mu \mathrm{L}$ Li-ATP (100 mM, Roche) and further incubated at $37^{\circ} \mathrm{C}$ for $20 \mathrm{~min}$. The beads were washed once with $400 \mu \mathrm{L}$ Wash Buffer 1 , three times with $400 \mu \mathrm{L} 1 \times$ PNK buffer, with centrifugation steps at $500 \mathrm{~g}$ for $5 \mathrm{~min}$ at $4^{\circ} \mathrm{C}$ between each wash. Before on-bead ligation of the $5^{\prime}$ adaptor, the beads were resuspended in $80 \mu \mathrm{L}$ 5' linker-mix (1.25 $\mu \mathrm{M}$ RNA5 ( $\left.{ }^{5}\right)$ containing 5' Linker, $1 \mathrm{mM}$ ATP (NEB), $60 \mathrm{U}$ RNasin, $40 \cup \mathrm{T} 4$ ssRNA ligase I (New England Biolabs), $1 \times \mathrm{PNK}$ buffer) at $16^{\circ} \mathrm{C}$ overnight. After the ligation reaction, the beads were washed with $400 \mu \mathrm{L}$ Wash Buffer $2(50 \mathrm{mM}$ Tris $/ \mathrm{HCl} \mathrm{pH} 7.8,50 \mathrm{mM} \mathrm{NaCl}, 10 \mathrm{mM}$ Imidazole $\mathrm{pH} 8.0,0.1 \%$ NP-40, $5 \mathrm{mM}$ 
$\beta$-mercaptoethanol) until excess radioactivity was removed. Purified protein-RNA complexes were eluted from the Ni-NTA beads by incubating with $200 \mu \mathrm{L}$ elution buffer (50 mM Tris/HCl pH 7.8, $50 \mathrm{mM} \mathrm{NaCl}, 150 \mathrm{mM}$ imidazole $\mathrm{pH} 8.0,0.1 \% \mathrm{NP}-40,5 \mathrm{mM}$ $\beta$-mercaptoethanol) for $5 \mathrm{~min}$. The elution step was repeated twice (final elution volume of $400 \mu \mathrm{L}$ ). The eluate was supplemented with $2 \mu \mathrm{g}$ bovine serum albumin (BSA) and complexes were TCA precipitated as described in section (2.8). The dried pellet was resuspended in $30 \mu \mathrm{L} 1 \times$ NuPAGE buffer containing $50 \mathrm{mM}$ DTT (Invitrogen) and stored at $-20^{\circ} \mathrm{C}$.

Protein-RNA samples were separated by electrophoresis on a 4-12 \% Bis-Tris NuPAGE gradient gel (Invitrogen) using 1 x MES buffer (50 mM MES, $50 \mathrm{mM}$ Tris-Base, $0.1 \%$ SDS, $1 \mathrm{mM}$ EDTA, pH 7.3) to prevent RNA hydrolysis. After $1.5 \mathrm{~h}$ electrophoresis at $100 \mathrm{~V}$, complexes were transferred by wet electroblotting to a HyBond $\mathrm{C}$ nitrocellulose membrane (GE Healthcare) at $80 \mathrm{~V}$ for $2 \mathrm{~h}$ at $4^{\circ} \mathrm{C}$. Once dried, the membrane was exposed to an $\mathrm{X}$ ray film using a Hyperscreen intensifying screen (GE Healthcare) for $1-3 \mathrm{~h}$ at $-80^{\circ} \mathrm{C}$ for detection of radioactive signal, and the corresponding region was cut out of the membrane. The membrane slice was subjected to protein digestion in $400 \mu \mathrm{L}$ Wash buffer 2 (containing $1 \%$ SDS and $5 \mathrm{mM}$ EDTA) and $6.5 \mu \mathrm{L}$ commercial proteinase $\mathrm{K}$ (Roche) at $55^{\circ} \mathrm{C}$ overnight. After the proteinase treatment, the RNA was extracted by mixing the solution with $50 \mu \mathrm{L} 3 \mathrm{M}$ sodium acetate $\mathrm{pH} 5.2$ and $500 \mu \mathrm{L} \mathrm{PCl}$, followed by phase separating centrifugation at $20,000 \mathrm{~g}$ for $5 \mathrm{~min}$. The upper phase was transferred to a new tube, mixed with $20 \mu \mathrm{g}$ glycogen and the RNA was precipitated with $1 \mathrm{~mL} 100 \%$ ethanol at $-20^{\circ} \mathrm{C}$ overnight. The suspension was centrifuged at $20,000 \mathrm{~g}$ for $30 \mathrm{~min}$. The obtained RNA pellet was washed with $500 \mu \mathrm{L} 70 \%$ ethanol and dried.

cDNA was prepared from the purified RNA fragments by resuspending the RNA pellet in $13 \mu \mathrm{L}$ RT-mix containing 0.8 $\mu \mathrm{M}$ RTP Primer and $0.8 \mathrm{mM}$ dNTP Mix (Roche). Primers were annealed to the RNA template by heating the sample to $80^{\circ} \mathrm{C}$ for $3 \mathrm{~min}$. The mixture was then kept on ice for $5 \mathrm{~min}$, supplemented with $4 \mu \mathrm{L} 5 \mathrm{x}$ first strand buffer (Invitrogen), $1 \mu \mathrm{L}$ $100 \mathrm{mM} \mathrm{DTT}, 1 \mu \mathrm{L} \mathrm{RNasin}(40 \mathrm{U} / \mu \mathrm{L})$, and incubated at $50^{\circ} \mathrm{C}$ for $3 \mathrm{~min}$. The reaction was then started by addition of $1 \mu \mathrm{L}$ SuperScript III reverse transcriptase (Invitrogen) and incubated for $1 \mathrm{~h}$ at $50^{\circ} \mathrm{C}$, followed by Superscript inactivation at $65^{\circ} \mathrm{C}$ for $15 \mathrm{~min}$. Finally, the RNA template was digested by treating the sample with $10 \mathrm{U}$ RNase $\mathrm{H}$ (New England Biolabs) at $37^{\circ} \mathrm{C}$ for 30 min. cDNAs were further amplified by PCR, in six independent reactions, using $1 \mu \mathrm{l}$ of RT-mix (Table 2.9).

Amplified DNA from 6 PCR reactions were mixed with $30 \mu \mathrm{L} 3 \mathrm{M} \mathrm{NaAc} \mathrm{pH} 5.2$, extracted with $1: 1$ ratio of $\mathrm{PCl}$ and precipitated with 3 volumes of $100 \%$ ethanol at $-20^{\circ} \mathrm{C}$ overnight. DNA was pelleted by centrifugation at $20,000 \mathrm{~g}$ for $20 \mathrm{~min}$ at $4^{\circ} \mathrm{C}$, subsequently dried at 
$37^{\circ} \mathrm{C}$ and resuspended in $15 \mu \mathrm{L} \mathrm{MQ}-\mathrm{H}_{2} \mathrm{O}$ and $5 \mu \mathrm{L} 4 \times$ Qiagen Loading buffer. The DNA sample was loaded onto a $3 \%$ Metaphore agarose gel (Lonza) and separated by electrophoresis in $1 \times$ TBE buffer ( $89 \mathrm{mM}$ Tris/HCl, $89 \mathrm{mM}$ boric acid, $2 \mathrm{mM}$ EDTA) at $100 \mathrm{~mA}$. The gel was stained in an ethidium bromide bath $(1: 10,000$ dilution of $10 \mathrm{mg} / \mathrm{mL}$ $\mathrm{EtBr}$ ) for visualisation of the amplified DNA. Fragments of approx. 150-400 bp length were excised from the gel and purified using a Qiagen MINI elute kit following the manufacturer's protocol. The concentration was determined and the obtained cDNA library was then used for single-end deep sequencing (Illumina). The bioinformatic analysis of the sequencing data was performed using software packages from the pyCRAC software suite (Webb et al., 2014) and in-house pipeline available in the Bohnsack group.

Table 2.9 Conditions for PCR amplification of CDNA.

\begin{tabular}{|c|c|c|c|c|}
\hline Component & Final Concentration & Temperature & Time & Cycles \\
\hline $10 \times$ LA Taq buffer $+\mathrm{MgCl}_{2}$ & $1 x$ & $95^{\circ} \mathrm{C}$ & $2 \min$ & $x 1$ \\
\hline Fw Oligo & $0.4 \mu \mathrm{M}$ & $98^{\circ} \mathrm{C}$ & $30 \mathrm{sec}$ & \\
\hline Index Oligo & $0.4 \mu \mathrm{M}$ & $60^{\circ} \mathrm{C}$ & $40 \mathrm{sec}$ & $x 40$ \\
\hline dNTPs & $0.125 \mathrm{mM}$ & $68^{\circ} \mathrm{C}$ & $40 \mathrm{sec}$ & \\
\hline LA TakaRa Taq & $2.5 \mathrm{U}$ & $72^{\circ} \mathrm{C}$ & $5 \mathrm{~min}$ & $x 1$ \\
\hline Template cDNA & $1 \mu \mathrm{L}$ & & & \\
\hline $\mathrm{MQ}-\mathrm{H}_{2} \mathrm{O}$ & to $50 \mu \mathrm{L}$ & & & \\
\hline
\end{tabular}

\subsection{Recombinant protein expression in E. coli}

E. coli BL21 codon+ or pLysS strains were transformed with 20 ng plasmid DNA (see section 2.2.3 and Table 2.4), plated on LB Amp/Chl $(100 \mu \mathrm{g} / \mathrm{ml}$ ampicillin, $34 \mu \mathrm{g} / \mathrm{ml}$ chloramphenicol) and incubated overnight at $37^{\circ} \mathrm{C}$. Single colonies were used to inoculate $50 \mathrm{~mL}$ LB Amp/Chl medium. The suspension was incubated over night at $37^{\circ} \mathrm{C}$, shaking. Cultures grew to stationary phase and were subsequently diluted in 1-6 L of LB Amp/Chl to an $\mathrm{OD}_{600}=0.1$ and further grown until $\mathrm{OD}_{600}=0.6$ was reached. Cultures were then cooled for $30 \mathrm{~min}$ at $4^{\circ} \mathrm{C}$ and protein expression was induced by addition of $1 \mathrm{mM}$ IPTG. Cultures were incubated overnight at $16^{\circ} \mathrm{C}$ while shaking. The following day, $0.1 \mathrm{mM}$ PMSF was added to the cultures and cells were harvested by centrifugation at $6,000 \mathrm{~g}$ for $20 \mathrm{~min}$ at $4^{\circ} \mathrm{C}$. Cell pellets were washed once with $10 \mathrm{~mL} 1 \times$ PBS buffer and centrifuged 
at $4,500 \mathrm{~g}$ for $10 \mathrm{~min}$ at $4^{\circ} \mathrm{C}$. Supernatants were discarded and cell pellets were stored at $-80^{\circ}$ or immediately used for protein purification.

\subsection{Recombinant Protein Purification}

For purification of recombinantly expressed proteins, a standard purification protocol for His-tag proteins with Ni-NTA beads was used. Cell pellets were resuspended in $20 \mathrm{~mL}$ of lysis buffer (50 mM Tris/ $\mathrm{HCl} \mathrm{pH} 7.4,500 \mathrm{mM} \mathrm{NaCl}, 1 \mathrm{mM} \mathrm{MgCl}, 5 \mathrm{mM}$ imidazole, $1 \mathrm{mM}$ PMSF, $10 \%$ glycerol) and cells were disrupted by sonication 4 times for $30 \mathrm{sec}$ at $45 \%$ amplitude ( $0.7 \mathrm{sec}$ on, $0.3 \mathrm{sec}$ off) on ice. The lysate was cleared by centrifugation at $20,000 \mathrm{~g}$ for $20 \mathrm{~min}$ at $4^{\circ} \mathrm{C}$. In order to remove nucleic acids and nucleic acid-associated proteins, polyethyleneimine ( $\mathrm{PEI}$, Sigma Aldrich) was added to the lysate to a final concentration of $0.05 \%(\mathrm{~V} / \mathrm{v})$ and incubated at $4^{\circ} \mathrm{C}$ for $15 \mathrm{~min}$, rotating. Afterwards, the lysate was again centrifuged at $33,000 \mathrm{~g}$ for $30 \mathrm{~min}$ at $4^{\circ} \mathrm{C}$.

$0.5 \mathrm{~mL}$ Ni-NTA matrix ( $1 \mathrm{~mL}$ bead slurry, Roche) were equilibrated by washing the matrix three times with $10 \mathrm{~mL}$ Wash Buffer I ( $50 \mathrm{mM}$ Tris/ $\mathrm{HCl} \mathrm{pH} \mathrm{7.4,500} \mathrm{mM} \mathrm{NaCl,} 1 \mathrm{mM} \mathrm{MgCl}$, $30 \mathrm{mM}$ imidazole, $10 \%$ glycerol) and centrifuging at $500 \mathrm{~g}$ for $1 \mathrm{~min}$ in between washes. The equilibrated Ni-Matrix was then incubated with the previously cleared lysate for $1 \mathrm{~h}$ rotating at $4^{\circ} \mathrm{C}$ and afterwards, the suspension was transferred to a column. The flowthrough (lysate) was collected and passed over the beads twice more. The beads were then washed once with $10 \mathrm{~mL}$ Wash Buffer I, once with $10 \mathrm{~mL}$ Wash Buffer II $(50 \mathrm{mM}$ Tris/ $\mathrm{HCl} \mathrm{pH} \mathrm{7.4,} 1 \mathrm{M} \mathrm{NaCl}, 1 \mathrm{mM} \mathrm{MgCl}$, $30 \mathrm{mM}$ imidazole, $10 \%$ glycerol) and once again with $10 \mathrm{~mL}$ Wash Buffer I.

Bound proteins were eluted by incubating the matrix with $3 \mathrm{~mL}$ Elution Buffer $(50 \mathrm{mM}$ Tris/ $\mathrm{HCl} \mathrm{pH} \mathrm{7.4,} 500 \mathrm{mM} \mathrm{NaCl}, 1 \mathrm{mM} \mathrm{MgCl} 2,300 \mathrm{mM}$ imidazole, $10 \%$ glycerol) for $5 \mathrm{~min}$ in the column. Following incubation, $1 \mathrm{~mL}$ fractions were collected. This procedure was repeated twice to obtain a total of 9 elution fractions. The protein content of each elution fractions was tested by spotting $1 \mu \mathrm{L}$ of each fraction onto a nitrocellulose membrane and staining with Amidoblack solution $(0.1 \%(\mathrm{w} / \mathrm{v})$ in $50 \%$ ethanol). Peak fractions containing most protein were pooled together and glycerol was added to a final concentration of $20 \%$. The protein solution was dialysed overnight against Dialysis Buffer $(50 \mathrm{mM}$ Tris/ $\mathrm{HCl}$ $\mathrm{pH} 7.4,120 \mathrm{mM} \mathrm{NaCl}, 2 \mathrm{mM} \mathrm{MgCl} 2,20 \%$ glycerol) in a dialysis tube (Spectrum Laboratories Inc) with an appropriate cut-off (less than half the molecular weight of the protein). 
Purified proteins were frozen in liquid nitrogen and stored at $-80^{\circ} \mathrm{C}$. To assess the purity of each fraction, samples were resolved by SDS-PAGE and Coomassie stained as described in section 2.8 and 2.9 .

\subsection{In vitro NADH-coupled ATPase assay}

To test the RNA-dependent ATPase activity of recombinantly expressed and purified proteins, an $\mathrm{NADH}$-coupled ATPase assay was performed as previously described (Kiianitsa et al., 2003). In short, $1.5 \mu \mathrm{M}$ protein samples were resuspended in $150 \mu \mathrm{L}$ reaction volume containing $50 \mathrm{mM}$ Tris/ $\mathrm{HCl} \mathrm{pH} \mathrm{7.4,} 25 \mathrm{mM} \mathrm{NaCl}, 2 \mathrm{mM} \mathrm{MgCl}$, $450 \mu \mathrm{M} \beta$ nicotinamide adenine dinucleotide (NADH), $4 \mathrm{mM}$ ATP, $1.5 \mathrm{mM}$ phosphoenolpyruvate (PEP), $20 \mathrm{U} / \mathrm{mL}$ pyruvate kinase/ lactic dehydrogenase (PK/LDH, Sigma-Aldrich) and either increasing amounts $(0-2 \mu \mathrm{M})$ or a single concentration of RNA (32 nt, 5'-GUAAUGCAAGUGAACGUACACACACACACACA-3'). Under these conditions, the hydrolysis of ATP and increased levels of ADP lead to the conversion of PEP into pyruvate by the supplemented pyruvate kinase enzyme. Pyruvate can then be converted into lactate by the $\mathrm{LDH}$ enzyme in a reaction coupled to NADH oxidation to NAD+. This reaction can be followed by measuring the decrease of absorption at $340 \mathrm{~nm}\left(\mathrm{~A}_{340}\right)$, the absorbance maximum of $\mathrm{NADH}$. The change in $\mathrm{A}_{340}$ was measured for $30 \mathrm{~min}$ in $50 \mathrm{sec}$ intervals in a BioTEK Synergy plate reader. The rates of ATP hydrolysis were calculated according to (Kiianitsa et al., 2003) as follows:

$$
\text { nM ATP hydrolysed } \times \sec ^{-1}=-\frac{d A_{340}}{d t} \times K_{\text {path }}^{-1} \times 10^{6}
$$

The molar absorption coefficient for a given optical pathlength $\left(K_{\text {path }}=2.1515 \mathrm{mM}^{-1}\right)$ was specified by the fill of $150 \mu \mathrm{L}$ per well and the background NADH decomposition.

\subsection{Anisotropy Measurements}

The RNA binding affinity of recombinantly expressed and purified proteins was measured by fluorescence anisotropy using a FluoroMax-4 spectrofluorometer (Horriba Scientific).

Proteins samples were first dialysed against Anisotropy Buffer $(30 \mathrm{mM}$ Tris/ $\mathrm{HCl} \mathrm{pH} \mathrm{7.5,}$ $50 \mathrm{mM} \mathrm{NaCl}$ ) overnight. In a final reaction volume of $120 \mu \mathrm{L}$, increasing concentrations of protein were incubated with $20 \mathrm{nM}$ of an Atto647-labelled RNA (5'GUAAUGAAAGU/3Atto647NN-3') at RT for $1 \mathrm{~min}$, and afterwards transferred into a Quartz SUPRASIL ${ }^{\circledR} 10 \times 2$ High Precision Cell cuvette (Hellma Analytics) for measurement. Anisotropy values were measured for protein concentrations ranging from 
$100 \mathrm{mM}$ to $4 \mu \mathrm{M}$ at $25^{\circ} \mathrm{C}$. Using the FluorEssence V3.5 software, single point anisotropy measurements were taken using excitation and emission wavelengths of $470 \mathrm{~nm}$ and $517 \mathrm{~nm}$, respectively, excitation slit width of $5 \mathrm{~nm}$, and emission slit width of $10 \mathrm{~nm}$. A maximal of six trials per sample was set, and a target standard error of $5 \%$. For each data point, the mean and the standard deviation of three independent measurements were calculated and plotted as a function of the concentration of protein used in each experiment. Dissociation constants were determined using the GraphPad Prism 6 software to fit the data.

\subsection{Binding assay of recombinantly expressed proteins}

\subsubsection{Binding assay using yeast lysate and purified recombinant proteins}

In vitro binding experiments were performed to assess interactions between Fal1 and Sgd1, or Rok1 and Sgd1. Sgd1 served as bait protein and was recombinantly expressed in E. coli and purified as described above (section 2.13 and 2.14). HA-tagged Fal1 and Rok1 were retrieved from crude yeast lysates. For each binding experiment $60 \mu \mathrm{l}$ of amylose resin (New England BioLabs) was equilibrated in Yeast Lysis Buffer (50 mM Tris/ $\mathrm{HCl} \mathrm{pH} \mathrm{7.8,} 150 \mathrm{mM} \mathrm{NaCl}, 1.5 \mathrm{mM} \mathrm{MgCl}_{2}, 0.05 \%$ NP-40, $5 \mathrm{mM} \beta$-mercaptoethanol, $+\mathrm{PI}$ ), by washing the matrix three times with $1 \mathrm{~mL}$ yeast lysis buffer. In between washing steps, the matrix was centrifuged at $100 \mathrm{~g}$ for $30 \mathrm{sec}$ at $4^{\circ} \mathrm{C} .1 \mathrm{~L}$ yeast cultures were grown until mid-log phase, harvested by centrifugation at 4,500 $\mathrm{g}$ and resuspended in one pellet volume of Yeast Lysis Buffer. Cells were then disrupted by glass bead lysis by incubating the cell suspension with $1.5 \mathrm{~mL}$ glass beads. Samples were thoroughly vortexed for $5 \mathrm{~min}$, $1.5 \mathrm{~mL}$ yeast lysis buffer was added and lysates were cleared from the glass beads by centrifugation at $4,000 \mathrm{~g}$ for $10 \mathrm{~min}$ at $4^{\circ} \mathrm{C}$. The supernatant was transferred into $1.5 \mathrm{~mL}$ tubes and cleared from cell debris by centrifugation at $20,000 \mathrm{~g}$ for $10 \mathrm{~min}$ at $4^{\circ} \mathrm{C} .1 \mathrm{~mL}$ cleared lysate was further treated with $30 \mu \mathrm{g}$ RNase A at $16^{\circ} \mathrm{C}$ for 15 min ("+ RNase" lysate). The remaining sample was directly used for the binding experiments ("- RNase" lysate). For each binding experiment, $60 \mu$ of equilibrated beads were first incubated with 300 pmol MBP-Sgd1-His 10 , MBP only (resuspended to $150 \mu$ in yeast lysis buffer), or the buffer alone, for $1 \mathrm{~h}$ at $4^{\circ} \mathrm{C}$. The beads were subsequently washed three times with $1 \mathrm{~mL}$ yeast lysis buffer and sedimented between each wash step as described above. The washed beads were added to $1 \mathrm{~mL}$ of RNase treated or untreated yeast lysate and incubated for $2 \mathrm{~h}$ at $4^{\circ} \mathrm{C}$, rotating. Afterwards, the matrix was washed five times as described above and transferred to a Mobicol column. Excess buffer was removed by centrifugation at $100 \mathrm{~g}$ for 30 seconds and the beads were resuspended in $100 \mu$ yeast 
lysis buffer containing $1.6 \mu \mathrm{g}$ TEV-protease, followed by overnight incubation at $4^{\circ} \mathrm{C}$, rotating. Afterwards, the elution fractions were collected by centrifugation (100 g, $30 \mathrm{sec}$, $4^{\circ} \mathrm{C}$ ). The proteins were precipitated with TCA, resolved by SDS-PAGE and visualised by immunoblotting as described in section 2.8.

\subsubsection{Binding assay using purified recombinant proteins}

To identify the Sgd1 regions important for binding to Fal1, recombinant $\mathrm{His}_{10}$ and MBPtagged full length Sgd1 and Sgd1 fragments were used as baits and incubated with recombinantly expressed His-Fal1.

Per sample, $60 \mu \mathrm{L}$ amylose matrix slurry were equilibrated by washing three times with $500 \mu \mathrm{L}$ binding buffer $\left(50 \mathrm{mM}\right.$ Tris/ $\mathrm{HCl} \mathrm{pH} \mathrm{7.8,} 300 \mathrm{mM} \mathrm{NaCl}, 1.5 \mathrm{mM} \mathrm{MgCl}_{2}, 0.1 \% \mathrm{NP}$ $40,1 \mathrm{mM} \mathrm{DTT},+\mathrm{Pl})$, centrifuging the matrix at $500 \mathrm{~g}$ at $4^{\circ} \mathrm{C}$ for $1 \mathrm{~min}$ in between washes. Beads were resuspended in $150 \mu \mathrm{L}$ binding buffer containing $300 \mathrm{pmol}$ MBP-tagged protein. Empty beads and tag-only controls were handled in the same way. Bead suspensions were supplemented with $10 \mu \mathrm{g}$ RNase $\mathrm{A}$ and incubated for $1 \mathrm{~h}$ at $4^{\circ} \mathrm{C}$ rotating. After the incubation step, the samples were centrifuged at $500 \mathrm{~g}$ for $1 \mathrm{~min}$ and the supernatant was discarded. The beads were washed five times with binding buffer as described before. Next, $150 \mu \mathrm{L}$ Binding buffer containing 300 pmol His-tagged Fal1 were added to all samples and incubated at $4^{\circ} \mathrm{C}$ for $2 \mathrm{~h}$ on a rotating wheel. The beads were again washed with binding buffer five times and transferred to a Mobicol column. The remaining buffer was centrifuged out of the column at $500 \mathrm{~g}$ for $1 \mathrm{~min}$ and the beads were resuspended in $100 \mu \mathrm{L} 1 \times \mathrm{SDS}$ buffer. Samples were boiled at $95^{\circ} \mathrm{C}$ for $5 \mathrm{~min}$ and the supernatant was separated from the beads by centrifugation at $500 \mathrm{~g}$ for $1 \mathrm{~min}$. Samples were separated by SDS-PAGE and visualised by Coomassie staining as described in section 2.8 . 


\section{Results}

\subsection{Depletion of Fal1 leads to pre-rRNA processing defects at cleavage sites}

\section{$A_{0}, A_{1}$ and $A_{2}$}

21 RNA helicases are involved in ribosome biogenesis in yeast, however, for several of them, their molecular function remains elusive. More specifically, eight RNA helicases are required for SSU biogenesis, a very dynamic process that is initiated with the formation of an SSU processome and the final steps of which occur in the cytoplasm. A largely uncharacterised RNA helicase involved in this pathway is Fal1. Fal1 is a DEAD-box helicase belonging to the elF4A-like subfamily of minimal helicases. Due to its close homology to elF4A, this protein was originally expected to have a role in translation initiation. However, Fal1 is not a functional homologue of elF4A and displays a nucleolar localisation. An early functional study of this protein revealed that Fal1 is required for the nucleolar maturation of the SSU particle (Kressler et al., 1997), but the binding site and the particular aspects of pre-SSU biogenesis for which this helicase is required have remained unclear. Advances in biochemical techniques, as well as the recently available structures of early pre-SSU particles, represent important tools for obtaining a deeper understanding of the functional role of assembly factors in the maturation process of the small subunit. Nonetheless, none of these approaches has yet provided any more insights into the role of Fal1. To better understand the role of Fal1 in ribosome biogenesis, we conducted in vivo and in vitro functional analyses of this protein.

As Fal1 is essential for cell viability (Kressler et al., 1997), in vivo functional analyses were carried out by depleting yeast cells of Fal1 rather than generating a deletion strain. For the depletion of Fal1, we established the auxin-inducible degradation system (AID) system, in yeast strains as previously described by Morawska and Ulrich, 2013. In brief, proteins carrying a degron tag (AID tag) can be targeted for proteasomal degradation in the presence of the plant hormone auxin. Auxin mediates the interaction between the degron tag and an auxin receptor, the F-box protein transport inhibitor response 1 (TIR1), which leads to ubiquitination of the degron tag and degradation of the target protein by the proteasome. Yeast strains expressing TIR1 and Fal1-6xHA or Fal1-6xHA-AID were grown to exponential phase and treated with auxin (indole-3-acetic acid, IAA) for targeted depletion of Fal1 carrying an AID tag. As yeast cells lack an auxin-responsive system, no changes in the expression levels of Fal1-6xHA were expected. Cells were harvested before treatment $(t=0 \mathrm{~min})$ and at different timepoints after IAA treatment $(t=30 \mathrm{~min}$, $t=60 \mathrm{~min}, \mathrm{t}=90 \mathrm{~min}$ ). A control culture was treated with $100 \%$ ethanol, the solvent used to resuspend auxin, and cells were harvested after $90 \mathrm{~min}(-\mathrm{IAA})$. Total proteins were 
extracted by TCA precipitation and analysed by SDS-PAGE and western blotting to detect the HA tag, and, as a loading control, the abundant cytoplasmic protein, Pgk1 (Figure 3.1).

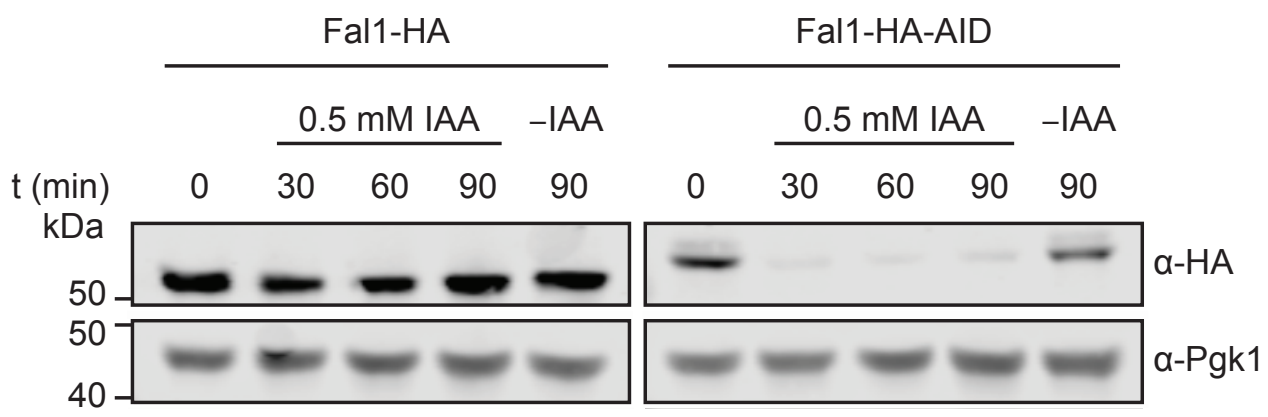

Figure 3.1 Auxin degron-based depletion of Fal1. Yeast strains expressing HA-tagged (Fal1$\mathrm{HA}$ ) or degron tagged (Fal1-HA-AID) Fal1 were treated with $0.5 \mathrm{mM}$ IAA for 30, 60 or $90 \mathrm{~min}$, or left untreated $(t=0$ and $-I A A)$. Total proteins were extracted and detected by western blotting using antibodies against the HA epitope or endogenous Pgk1 as a loading control.

As expected, we did not observe a reduction of Fal1-HA protein levels after auxin treatment, indicating a lack of auxin-induced unspecific degradation. This finding also confirms that the stability of Fal1-HA is not significantly affected by introducing a C-terminal HA-tag onto the protein. Intriguingly, we detected slightly lower basal levels of Fal1-HAAID than those of Fal1-HA before treatment. This could be due to the degron-tag affecting the stability of the protein. Importantly, we observed that auxin treatment lead to a reduction of Fal1-HA-AID protein levels after $30 \mathrm{~min}$, and until 90 min after treatment. To allow enough time for the effects of Fal1 depletion in ribosome biogenesis to be detectable, an optimal depletion time for the functional analysis of Fal1 was established at 60 min after auxin treatment.

Fal1 has been shown to be necessary for maturation of the 18S rRNA precursor (Kressler et al., 1997). To identify the precise pre-rRNA processing events that require Fal1, we analysed the processing of pre-rRNA in Fal1-depleted cells by northern blotting. Yeast strains expressing Fal1-HA or Fal1-HA-AID were grown to exponential phase and subjected to IAA treatment for 60 min. RNA was extracted, separated by denaturing gel electrophoresis and pre-rRNA species were detected using radioactively labelled oligonucleotide probes (004 and 020), which base-pair to the internal transcribed spacer sequences (Figure 3.2A). 
A

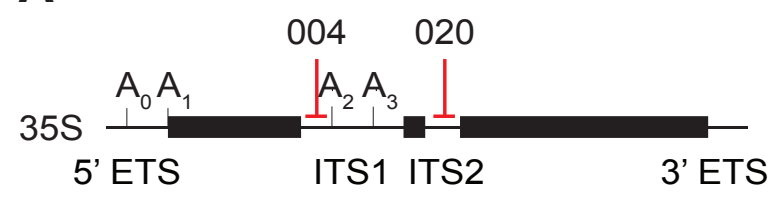

$32 S$

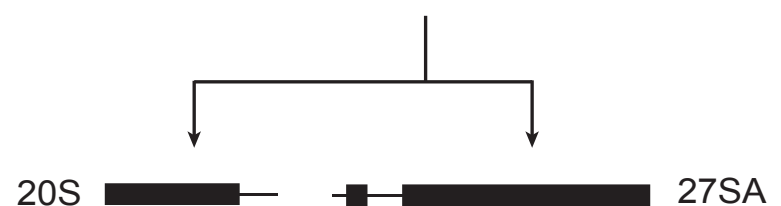

205

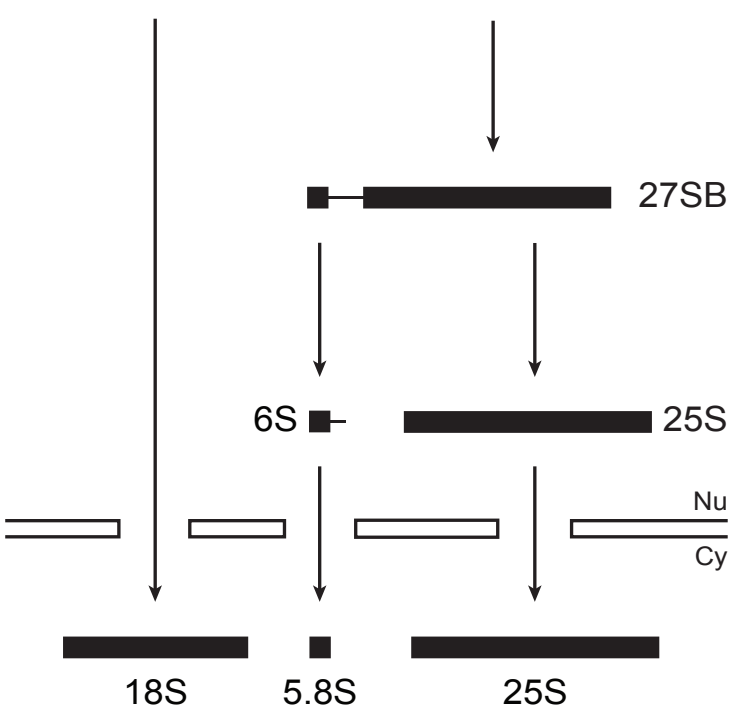

B
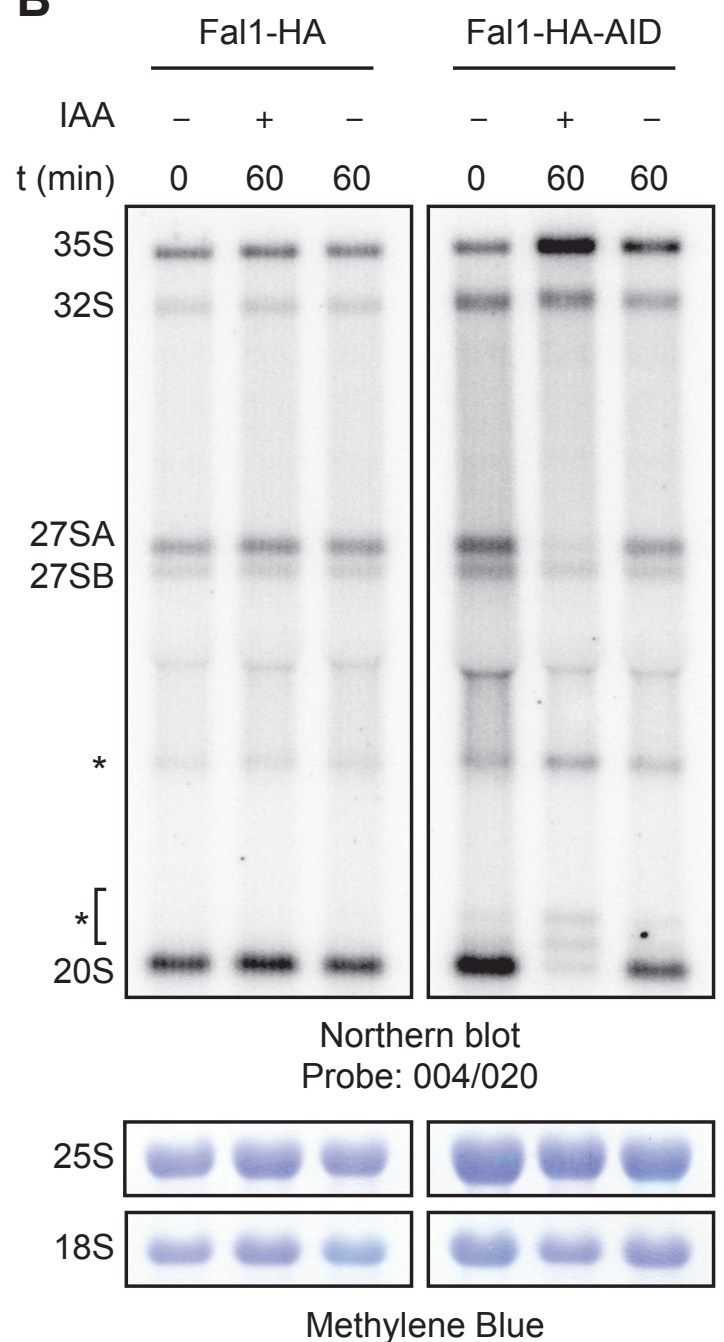

Figure 3.2 Depletion of Fal1 leads to SSU pre-rRNA processing defects. (A) Simplified prerRNA processing scheme. Selected cleavage sites are indicated on the 35S pre-rRNA. Processing steps occurring in the nucleus $(\mathrm{Nu})$ or cytoplasm (Cy) are separated by white bars. The binding regions of the radiolabelled oligonucleotide probes (004 and 020) used for northern blot detection of rRNA precursors are indicated in red on the 35S pre-rRNA (for more detailed information see Table 2.8). (B) Northern blot showing the pre-rRNA processing intermediates present in yeast strains untreated (-IAA) or treated with $0.5 \mathrm{mM}$ IAA for $60 \mathrm{~min}$. Mature 25S and 18S rRNA levels are shown by methylene blue staining. Aberrant precursors accumulated upon Fal1 depletion are indicated with an asterisk $\left({ }^{*}\right)$.

Consistent with the depletion of an 18S rRNA maturation factor, depletion of Fal1-HA-AID did not affect mature 25S rRNA levels, but led to a slight reduction of mature 18S rRNA (Figure 3.2B, methylene blue staining). Despite the mild effect observed on mature $18 \mathrm{~S}$ rRNA, the level of its immediate precursor, the $20 \mathrm{~S}$ pre-rRNA, was drastically reduced when Fal1-HA-AID was depleted. Depletion of the helicase also lead to accumulation of 
the 35S pre-rRNA, which indicates impaired co-transcriptional processing of the earliest pre-rRNA transcripts. Interestingly, the level of the 27SA pre-rRNA was also observed to be reduced upon Fal1-HA-AID depletion, but not the other 25S rRNA precursors, thus enabling production of mature 25S rRNA. Intriguingly, depletion of Fal1-HA-AID lead to the accumulation of aberrant rRNA precursors, which are not intermediates of the normal pre-rRNA processing pathway (indicated with an asterisk in Figure 3.2B).

The pre-rRNA processing defects observed upon Fal1 depletion suggest an impairment in early processing steps of pre-18S rRNA, whereas pre-25S rRNA processing was not significantly affected. An alternative pathway of pre-18S rRNA processing could explain the accumulation of aberrant precursors under depletion conditions (Figure 3.3A). Impaired cleavage at sites $A_{0}$ and $A_{1}$ within the 5 ' ETS sequence and $A_{2}$ within ITS1 lead to the production of aberrant 23S, 22S and 21S pre-rRNA species and thereby hindering $20 \mathrm{~S}$ pre-rRNA production and 18S rRNA maturation. To determine whether the aberrant species accumulated during Fal1 depletion correspond to these pre-rRNAs, oligonucleotide probes were designed that base-pair with different regions within the 5' ETS sequence and the ITS1, and were used for northern blot mapping of the aberrant precursors (Figure 3.3B).

Mapping of the aberrant pre-rRNAs showed that two of the unidentified pre-rRNA species include a sequence located between the $A_{0}$ and $A_{1}$ cleavage sites, indicating that in these two rRNA species, but not in the third one, cleavage at $A_{1}$ is impaired (Figure 3.3B, probe a). All three aberrant pre-rRNAs were found to contain the region between the $A_{2}$ and $A_{3}$ cleavage sites, consistent with impaired $A_{2}$ cleavage (Figure 3.3B, probe b). Interestingly, none of the aberrant pre-rRNAs were detected with a probe base-pairing to the sequence downstream of the $A_{3}$ cleavage site, suggesting effective $A_{3}$ cleavage of the nascent prerRNA upon Fal1 depletion (Figure 3.3B, probe c). Taken together, these data show that Fal1 depletion leads to the accumulation of two pre-rRNA species including intact $A_{1}$ and $A_{2}$ cleavage sites ( $21 S$ and $22 S$ pre-rRNAs), and one pre-rRNA species including a noncleaved $A_{2}$ site (23S pre-rRNA). These results are consistent with the model that depletion of Fal1 leads to alternative processing of $18 \mathrm{~S}$ rRNA precursors upon impaired $A_{0}, A_{1}$ and $\mathrm{A}_{2}$ cleavage. 
A

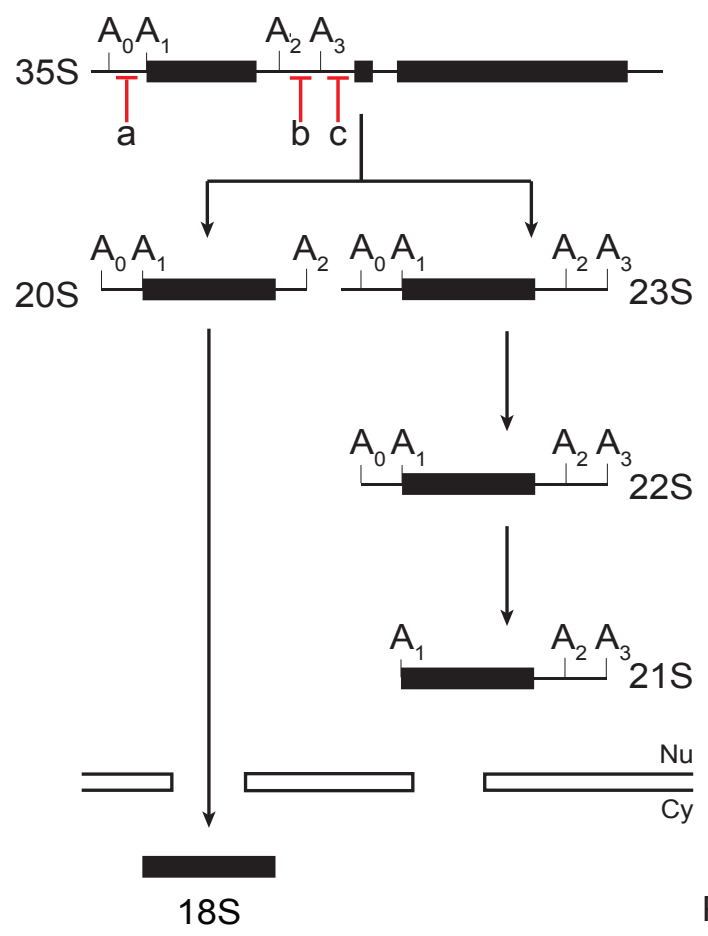

B

Fal1-HA-AID

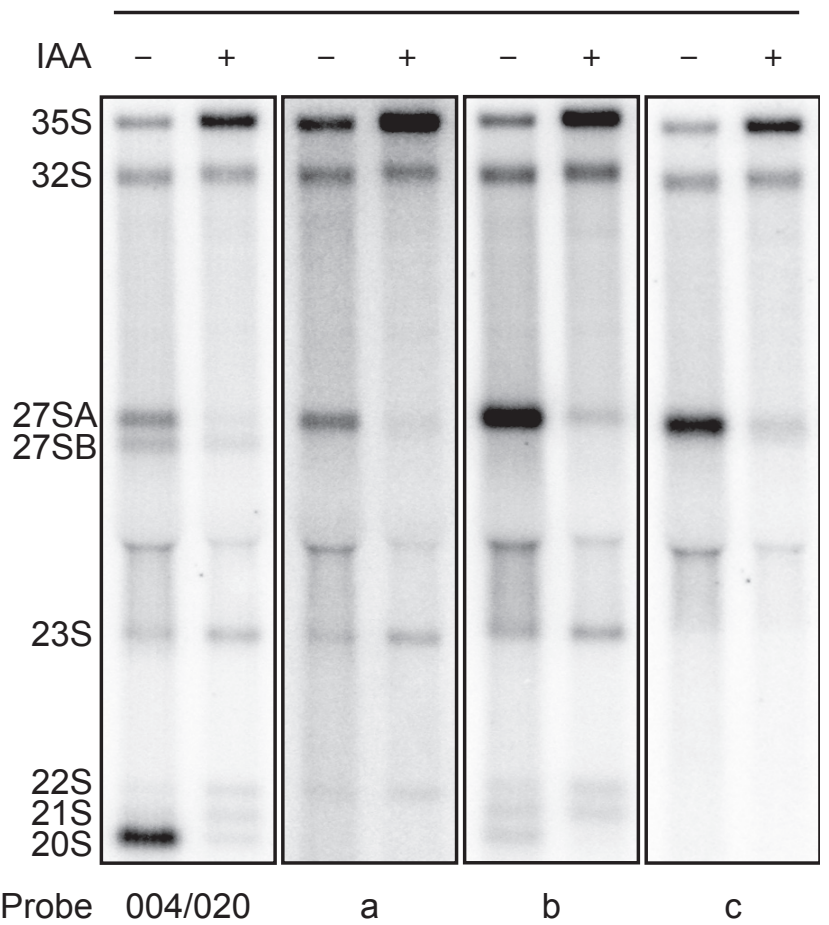

Figure 3.3 Depletion of Fal1 leads to accumulation of aberrant 23S, 22S and 21S pre-rRNAs. (A) Alternative pre-rRNA processing scheme for the pre-18S rRNA. Selected cleavage sites are indicated on the $35 \mathrm{~S}$ pre-rRNA. White bars illustrate the separation of processing events occurring in the nucleus $(\mathrm{Nu})$ or the cytoplasm (Cy). The binding regions of radiolabelled oligonucleotide probes $(a, b$ and $c$ ) used for mapping of pre-rRNAs are indicated in red on the $35 \mathrm{~S}$ pre-rRNA (for more detailed information see Table 2.8). (B) Northern blot showing the mapping of aberrant rRNA precursors from yeast cells left untreated $(-I A A)$ or depleted of Fal1 $(+\mid A A)$ using different radiolabelled probes.

\subsection{The ATPase activity of Fal1 is required for pre-rRNA processing}

Depletion experiments showed that Fal1 is required for correct processing of the SSU pre-rRNA. As Fal1 is an ATP-dependent RNA helicase (Banroques et al., 2011), the hypothesis that the pre-rRNA processing defects arise from the lack of ATP hydrolysis-dependent activity of Fal1 was addressed.

To study the role of the catalytic activity of Fal1 in ribosome biogenesis, a probable activity mutant was first generated and its ATPase activity was tested in vitro. Motif II of the helicase core, which contains the DEAD amino acid sequence, is proposed to be responsible for ATP binding and hydrolysis (Walker 1982), and mutations of this motif are expected to disrupt ATPase activity. To generate a DQAD-mutant version of Fal1, a singleamino acid substitution ( $E_{174}$ to $Q_{174}$ ) was introduced by site-directed mutagenesis of the 
DEAD sequence of motif II. Wild-type (WT) and DQAD-mutant Fal1 were recombinantly expressed in $E$. coli cells and subsequently affinity purified (Figure 3.4A). To test the ATPase activity of Fal1_WT and the Fal1_DQAD-mutant, an in vitro NADH-coupled ATPase assay was used in the presence and absence of RNA (Figure 3.4.B).

A

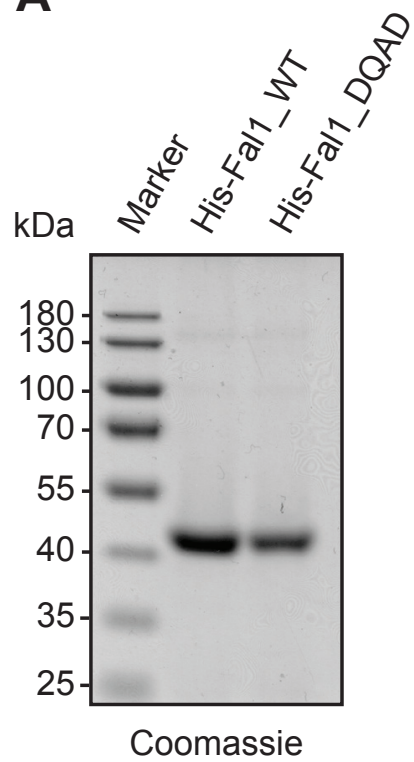

B

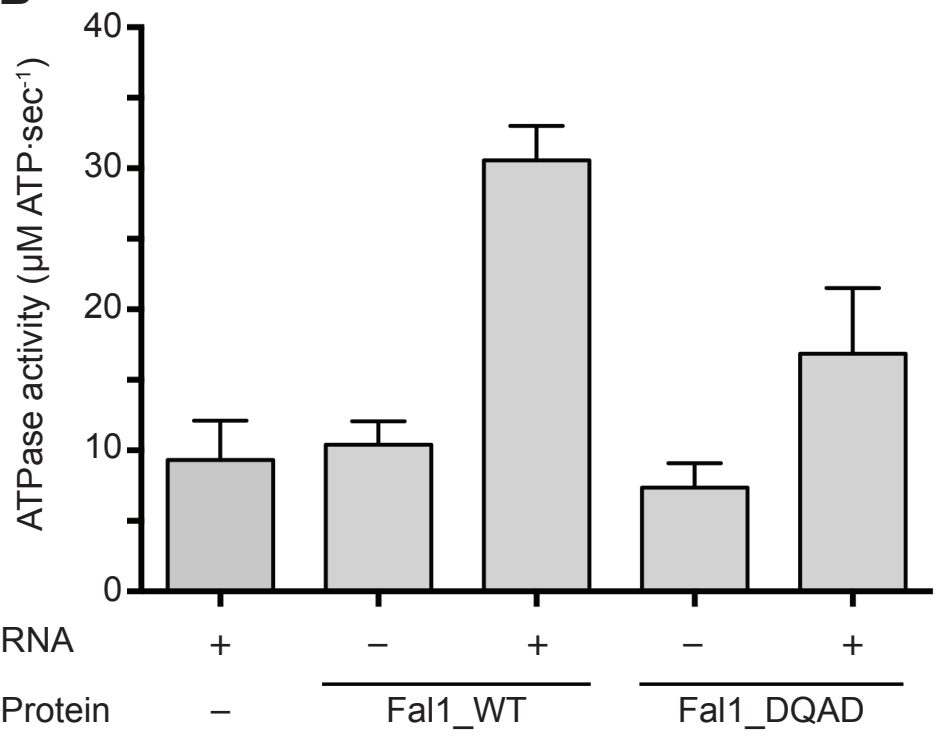

Figure 3.4 Fal1 is an RNA-dependent ATPase, and mutation of the DEAD sequence within Motif II impairs ATPase activity in vitro. (A) Coomassie stained SDS-PAGE gel of recombinantly expressed and affinity purified Fal1 WT and DQAD mutant. (B) ATPase activity of Fal1_WT and Fal1_DQAD measured by NADH-coupled ATPase assay with and without the addition of RNA. A negative control without protein shows background ATP turnover. The data of three independent experiments are presented as mean \pm standard deviation.

Under the experimental conditions, no ATP hydrolysis activity above background ATP turnover was detected for Fal1_WT in the absence of RNA in vitro (Figure 3.4B, no-protein control and Fal1_WT -RNA). Importantly, incubation of Fal1_WT with RNA significantly enhanced the ATPase activity of the helicase (Figure 3.4B, Fal1_WT +RNA). These results show that Fal1_WT is an RNA-dependent ATPase. The mutant Fal1_DQAD showed no ATPase activity above background without RNA present (Figure 3.4B, Fal1_DQAD -RNA). While addition of RNA also stimulated ATP hydrolysis by Fal1_DQAD (Figure 3.4B, Fal1_DQAD +RNA), this mutant showed impaired ATPase activity compared to Fal1_WT under the same conditions. These results demonstrate that the DQAD-mutant is a catalytic mutant of Fal1 that can be used for further functional characterisation of the helicase in vivo. 


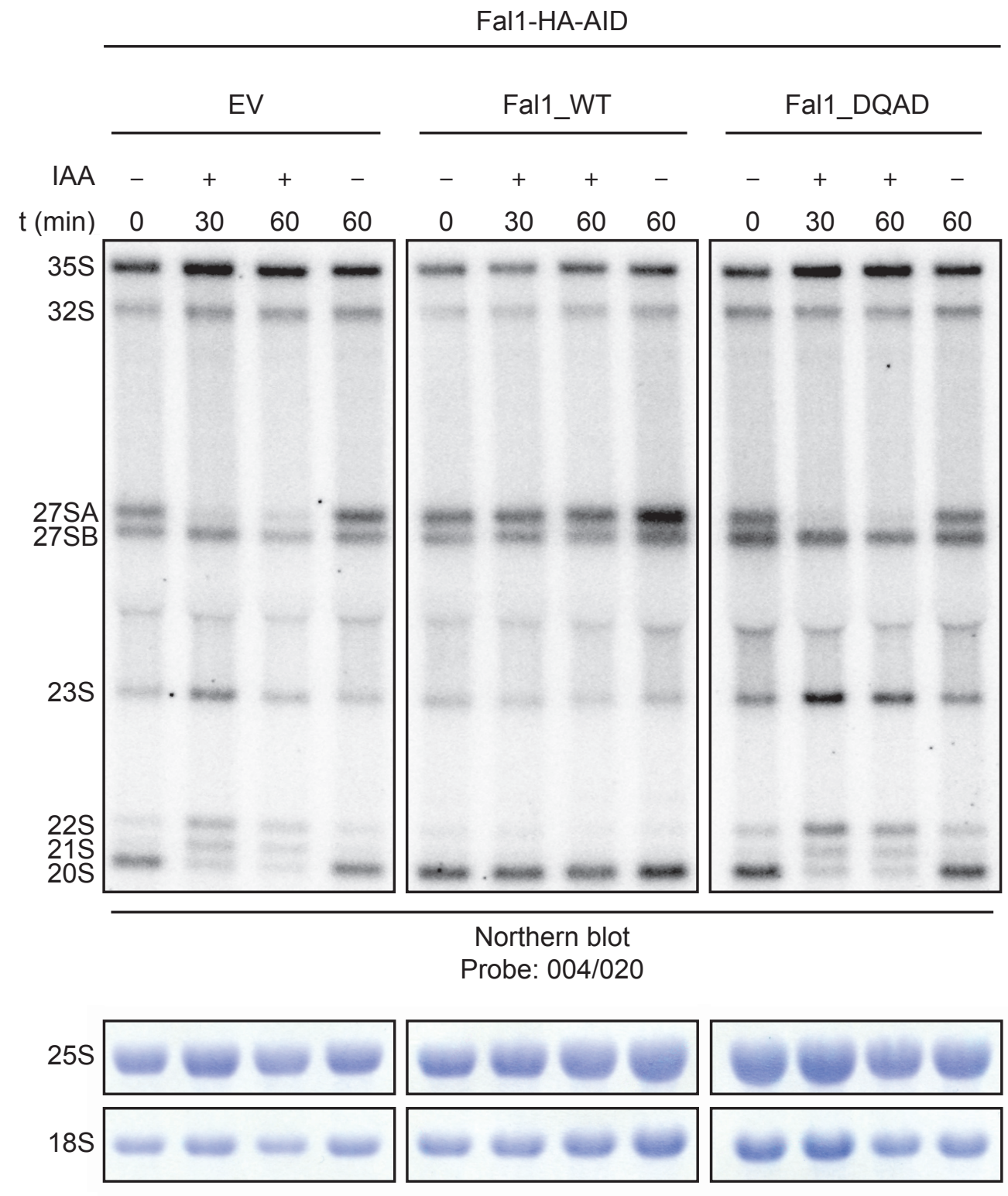

Methylene Blue

Figure 3.5 The ATPase activity of Fal1 is required for SSU pre-rRNA processing. Northern blot showing the pre-rRNA processing intermediates in yeast strains left untreated (-IAA) or depleted of Fal1 (+IAA) and transformed with an empty vector (EV), or with a vector encoding wild-type Fal1 (Fal1_WT) or an activity mutant (Fal1_DQAD). Methylene blue staining shows the mature 25S and 18S rRNAs.

To determine whether the ATPase activity of Fal1 is required for its role in ribosome biogenesis, an in vivo complementation assay was performed. The Fal1-depletion strain was transformed with an empty vector (EV) control or constructs allowing for exogenous expression of Fal1_WT or Fal1_DQAD. After growing the cells under depletion conditions, RNA was extracted and pre-rRNA processing was analysed by northern blotting to 
determine if the exogenously expressed proteins could complement the pre-rRNA processing defects (Figure 3.5).

After auxin treatment, the yeast strain transformed with EV showed the previously mentioned pre-rRNA processing defects corresponding to Fal1 depletion (Figure 3.5; EV, $\mathrm{t}=30$ and $60 \mathrm{~min},+\mathrm{IAA})$. Importantly, exogenous expression of Fal1_WT complemented the pre-rRNA processing defects caused by Fal1 depletion (Figure 3.5; Fal1_WT, $\mathrm{t}=30$ and $60 \mathrm{~min},+(\mathrm{AA})$. Interestingly, exogenous expression of catalytically inactive Fal1_DQAD did not rescue the pre-rRNA processing defects caused by Fal1 depletion (Figure 3.5; Fal1_DQAD, $\mathrm{t}=30$ and $60 \mathrm{~min},+\mathrm{IAA}$ ). These data show that the ATPase activity of Fal1 is required for correct cleavage of the pre-rRNA transcript at the processing sites $A_{0}, A_{1}$ and $A_{2}$.

\subsection{The MIF4G domain-containing protein Sgd1 interacts with Fal1 in vivo and depletion of Sgd1 leads to similar pre-rRNA processing defects as Fal1 depletion}

A genetic screen previously identified the nucleolar protein Sgd1 as a genetic interactor of Fal1 (Alexandrov et al., 2011). Furthermore, Sgd1 is an elF4G-like protein that carries the middle domain of elF4G (MIF4G domain) and a second elF4G HEAT domain, the MA3 domain (Alexandrov et al., 2011; Marintchev and Wagner, 2005; Ponting, 2000). A mutational analysis showed that Sgd1 and Fal1 are required for ribosome biogenesis, and it has been proposed that these two proteins form an elF4A/elF4G-like functional complex required for $18 \mathrm{~S}$ rRNA maturation (Alexandrov et al., 2011). Based on this hypothesis, a functional analysis of the role of Sgd1 in ribosome biogenesis was conducted. As Sgd1 is an essential protein (Akhtar et al., 2000), a yeast strain for the depletion of Sgd1 using the AID system was first generated (Figure 3.6). 


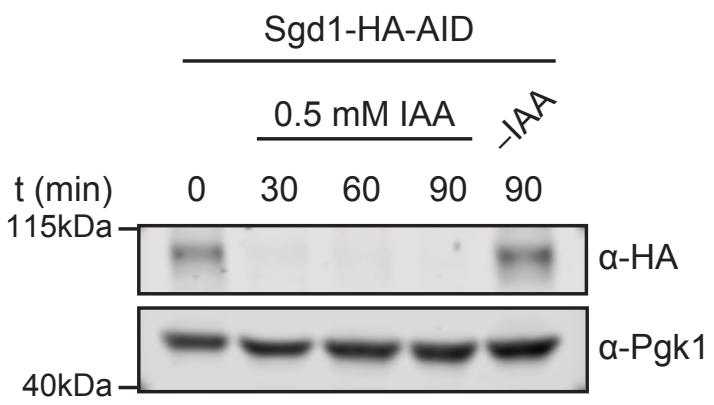

Figure 3.6 Auxin degron-based depletion of Sgd1. A yeast strain expressing degron tagged Sgd1 (Sgd1-HA-AID) was treated with $0.5 \mathrm{mM}$ IAA for $0,30,60$ or $90 \mathrm{~min}$, or left untreated (-IAA). Total cellular proteins were extracted and protein levels were detected by western blotting using antibodies against the HA epitope (Sgd1) or endogenous Pgk1 as loading control.

Efficient depletion of Sgd1-HA-AID was achieved within the first 60 min of auxin treatment, and longer auxin treatment did not increase the effect on the Sgd1 protein level. Therefore, a 60 min depletion time was used for further experiments to allow effects of Sgd1 depletion on ribosome biogenesis to be detectable.

To study the impact of Sgd1 depletion on ribosome biogenesis, we analysed pre-rRNA processing by northern blotting of RNAs derived from cells depleted of Sgd1-HA-AID (Figure 3.7A). Interestingly, depletion of Sgd1-HA-AID lead to a similar pre-rRNA processing phenotype as previously observed for Fal1 depletion (Figure 2.2B); decreased levels of 20S and 27SA pre-rRNAs and a concomitant accumulation of 35S pre-rRNA were observed. Importantly, depletion of Sgd1-HA-AID also led to accumulation of the aberrant precursors 23S, 22S and 21S, as shown by northern blot mapping of these RNA species using different radiolabelled probes (Figure 3.7B and C). These results support a shared role of Sgd1 and Fal1 in SSU pre-rRNA processing. 
A

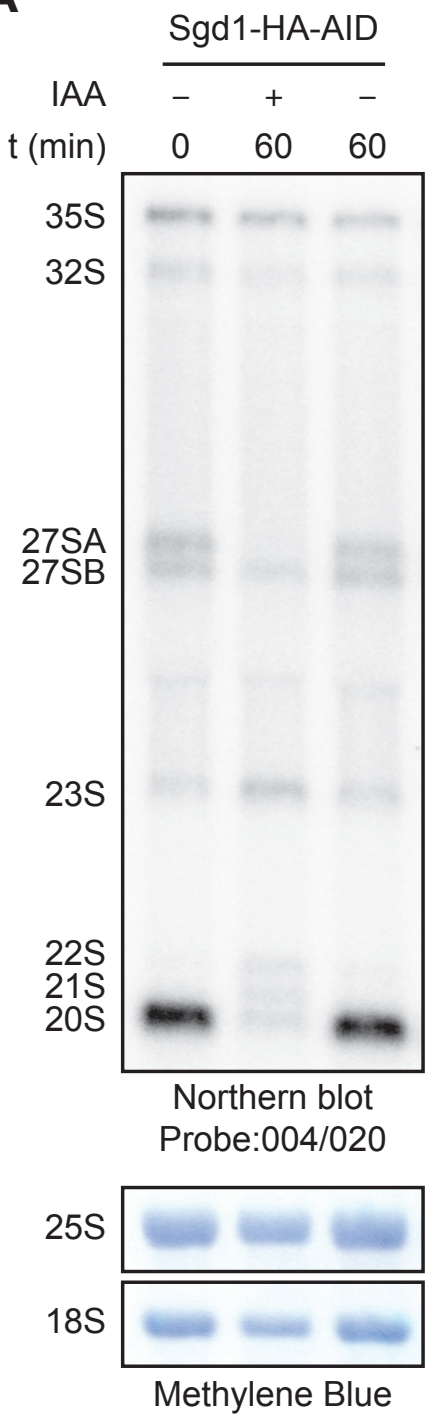

B

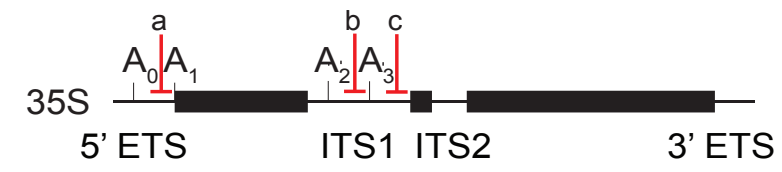

C

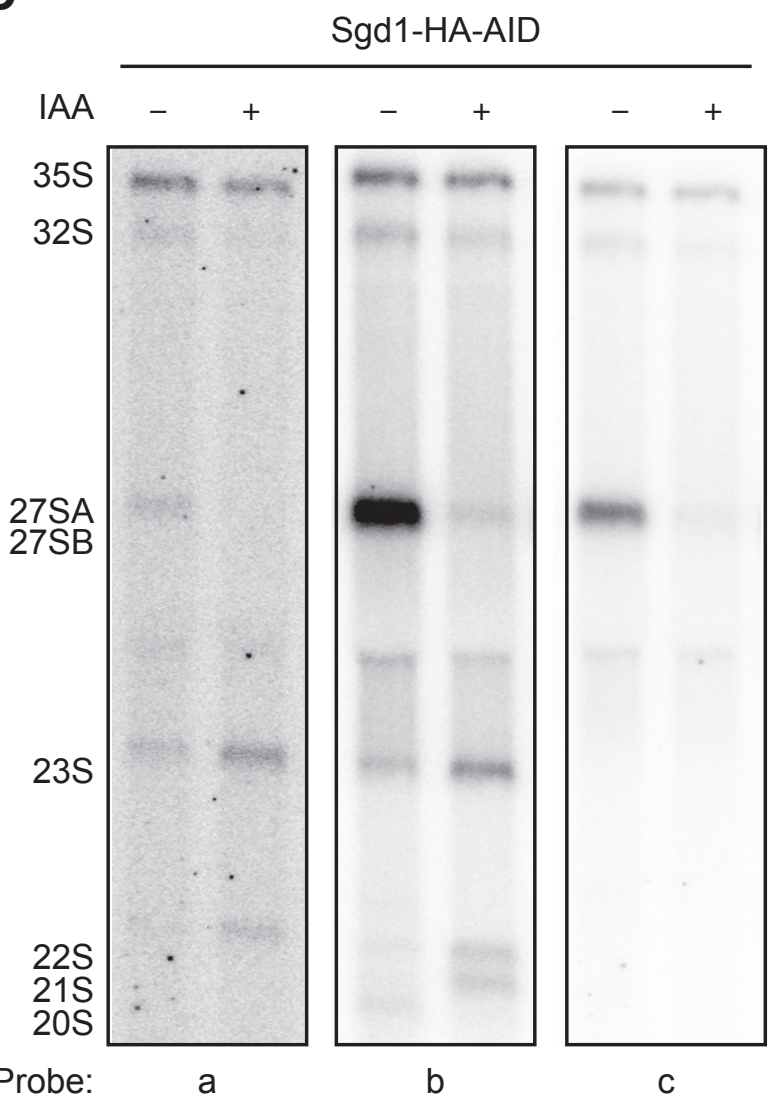

Figure 3.7 Depletion of Sgd1 leads to similar defects in pre-rRNA processing to Fal1 depletion. (A) Northern blot showing pre-rRNA intermediates present in yeast strains left untreated (-IAA) or depleted of Sgd1 (+IAA). (B) Scheme showing selected pre-rRNA cleavage sites and the binding sites on pre-rRNA (indicated in red) of radiolabelled oligonucleotide probes used for mapping rRNA precursors ( $a, b$ and $c$ ). (C) Northern blot showing the mapping of aberrant rRNA precursors accumulated upon depletion of Sgd1 using different radiolabelled probes.

The DEAD-box helicase elF4A and the cofactor protein elF4G are known to interact directly in vivo and act together in translation initiation (Hilbert et al., 2011; Schutz et al., 2008). Fal1 and Sgd1 are an elF4A-like helicase and an elF4G-like protein respectively, therefore the hypothesis that Fal1 and Sgd1 can form a complex in vivo was addressed. To do this, a Sgd1 variant carrying a N-terminal MBP-tag and a C-terminal His-tag was 
recombinantly expressed and affinity purified from E. coli (Figure 3.8). The purified protein was then employed in pull-down experiments.

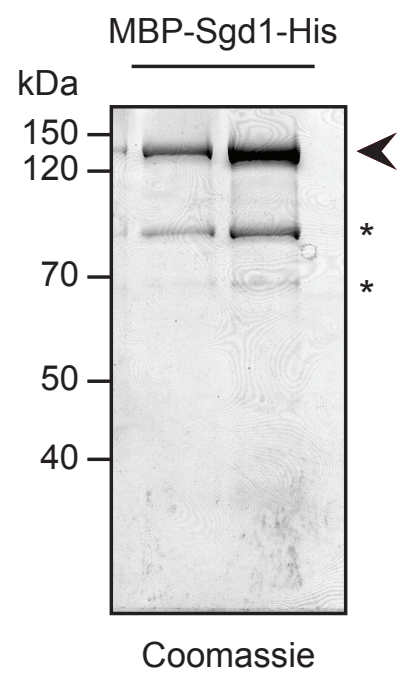

Figure 3.8 Recombinant expression and purification of Sgd1 from E. coli. Coomassie stained SDS-PAGE of recombinant MBP-Sgd1-His. Coomassie band corresponding to MBP-Sgd1-His is indicated with an arrow. Co-purified contaminants are indicated with an asterisk $\left({ }^{*}\right)$.

Recombinantly expressed MBP-Sgd1-His or MBP-His were immobilised on amylose beads and incubated with lysates prepared from yeast cells expressing HA-tagged Fal1 (HA-Fal1 lysate) or, as a control, cells expressing a HA-tagged version of another RNA helicase required for SSU biogenesis, Rok1 (HA-Rok1 lysate). To differentiate between RNA-mediated interactions and protein-protein interactions, the yeast lysates were treated with RNase A prior to incubation with the immobilised proteins (Figure 3.9). Despite treatment with protease inhibitors, and although MBP-Sgd1-His was recombinantly expressed and purified, incubation with yeast lysate resulted in significant cleavage of the bait protein, as detected by $\alpha-M B P$ antibody (Figure 3.9; MBP*). Consequently, a longer exposure time was required to detect full-length MBP-Sgd1-His in the elution fractions. 


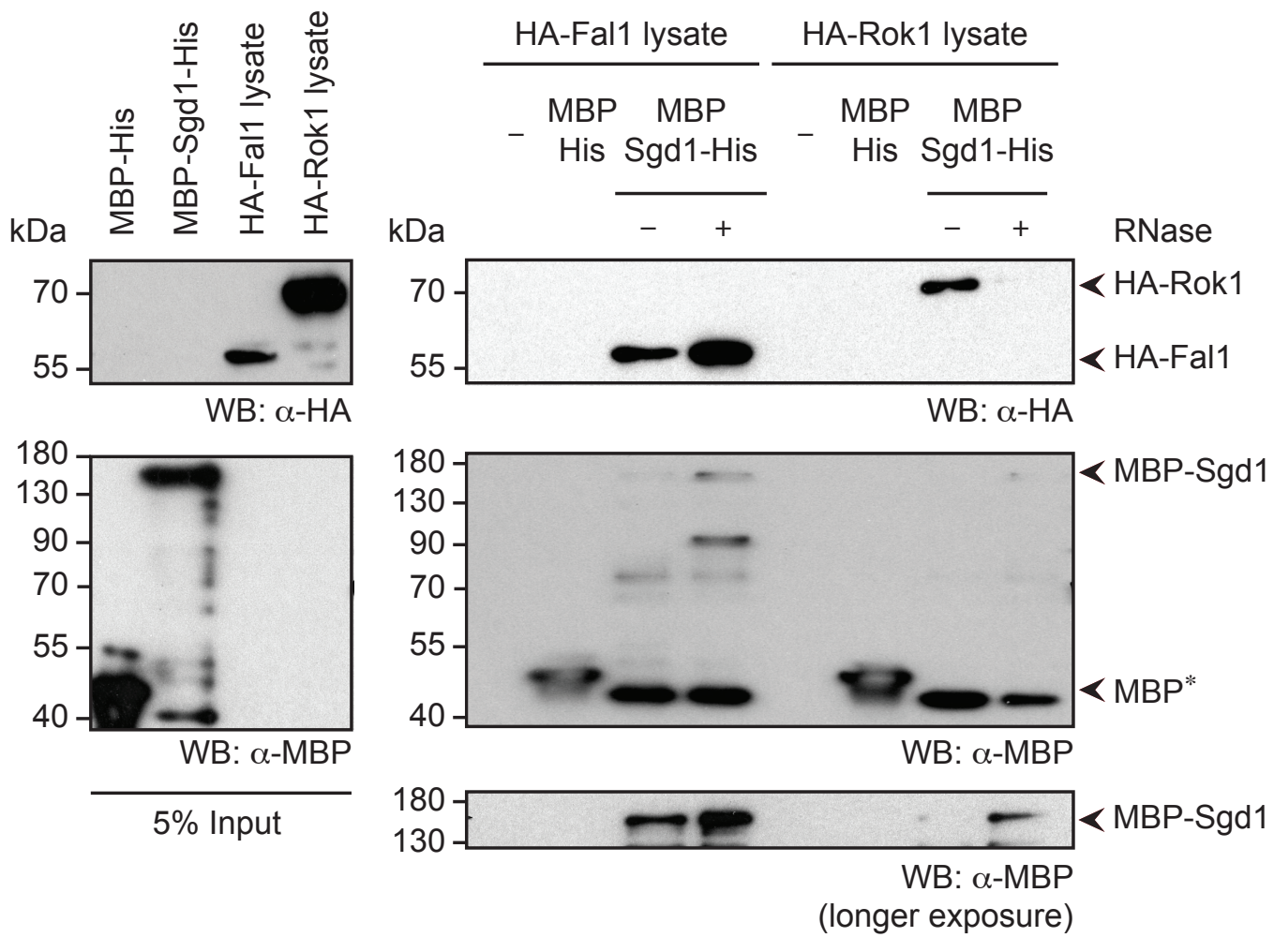

$20 \%$ Elution

Figure 3.9 Fal1 is retrieved from yeast cell extracts by Sgd1. Binding assay using immobilised, purified MBP-Sgd1-His or MBP-His, and yeast lysates prepared from cells expressing HA-Fal1 or HA-Rok1. Lysates were treated with RNase A (+ RNase) or left untreated (- RNase) prior to incubation with the immobilised bait proteins. Recovered proteins were detected by western blotting using antibodies against the HA epitope or the MBP-tag. A longer exposure is shown to visualize the signal detected for full length MBP-Sgd1 more clearly. The MBP tag and a degradation product of MBP-Sgd1 are indicated by MBP*.

As expected, no unspecific binding was detected for HA-Fal1 or HA-Rok1 in control samples incubated with empty beads (-) or the immobilized MBP-His control (MBP-His). Interestingly, both HA-Fal1 and HA-Rok1 were significantly enriched with immobilised MBP-Sgd1-His (Figure 3.9; -RNase). However, RNase treatment abolished the interaction between MBP-Sgd1-His and HA-Rok1, indicating that this interaction is RNA-dependent, and probably occurs in the context of a larger RNA-protein complex. Importantly, HA-Fal1 could be retrieved by immobilised MBP-Sgd1-His even after RNase treatment of the lysate. These results show that Fal1 and Sgd1 can associate in vivo in an RNA-independent manner. 


\subsection{The MIF4G domain of Sgd1 binds to Fal1 and can stimulate the ATPase activity of Fal1 in vitro}

Helicases of the elF4A-like subfamily, such as Fal1, are so-called minimal helicases that lack $\mathrm{N}$ - and $\mathrm{C}$-terminal extensions, and it is therefore suggested that other factors may induce their substrate specificity (Andreou and Klostermeier, 2013). It has been demonstrated that many MIF4G domain-containing proteins act as cofactors of elF4A-like helicases in various cellular processes (Buchwald et al., 2013; Montpetit et al., 2011; Schutz et al., 2008; Sloan and Bohnsack, 2018). Importantly, the conserved MIF4G domain and MA3 domain have been shown to be critical for the interaction with their corresponding helicase (Morino et al., 2000; Ponting, 2000; Schutz et al., 2008).

As Fal1 and Sgd1 are an elF4A/elF4G-like pair that are required for specific steps in ribosome biogenesis, and interact in an RNA-independent manner in vivo (Figure 3.9), we hypothesised that Sgd1 may bind to Fal1 through its MIF4G and/or MA3 domains and modulate the activity of the helicase. To address this hypothesis, in vitro binding assays using recombinantly expressed full-length and truncated proteins were performed, and the effect of Sgd1 on the ATPase activity of Fal1 was tested by in vitro ATPase assays. To determine which region of Sgd1 mediates the interaction with Fal1, N-terminally MBPand C-terminally His-tagged variants of Sgd1 were engineered produced and used in an in vitro protein-protein binding assay. As no structural information is available for Sgd1, a sequence alignment of elF4G-like proteins was performed to determine the predicted position of the MIF4G and MA3 domains and different truncation-constructs were created (Figure 3.10A).

Constructs for the recombinant expression of the $\mathrm{N}$-terminus and the predicted MIF4G

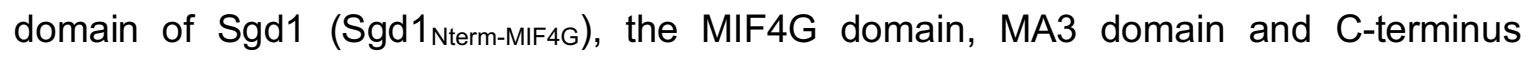

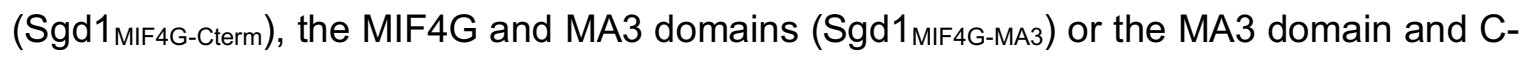

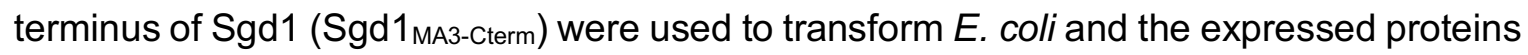
were affinity purified, as well as a full-length version of Sgd1 (Sgd1 ${ }_{\mathrm{FL}}$; Figure 3.10B). 
A

\begin{tabular}{|c|c|c|c|c|c|}
\hline MBP & & MIF4G & MA3 & His & MBP-Sgd $1_{F L}-H i s$ \\
\hline $\mathrm{MBP}$ & MIF4G & MA3 & & & MBP-Sgd $1_{\text {MIF } 4 \mathrm{G}-\mathrm{MA} 3}-\mathrm{HiS}$ \\
\hline $\mathrm{MBP}$ & MIF4G & MA3 & $\mathrm{His}$ & & MBP-Sgd1 $1_{\text {MIF } 4 \mathrm{G}-\mathrm{C} \text { term }}-\mathrm{His}$ \\
\hline $\mathrm{MBP}$ & & MIF4G & His & & MBP-Sgd $1_{\mathrm{N} \text { term-MIF } 4 \mathrm{G}}-\mathrm{His}$ \\
\hline $\mathrm{MBP}$ & \begin{tabular}{l|l|} 
MA3 \\
\end{tabular} & His & & & MBP-Sgd $1_{\text {MA3-C term }}-\mathrm{His}$ \\
\hline
\end{tabular}

B

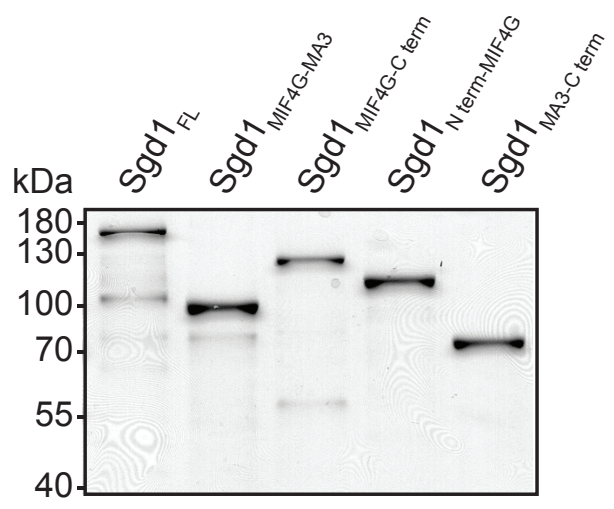

Coomassie

Figure 3.10 Recombinant expression and purification of full-length Sgd1 and Sgd1 fragments. (A) Schematic view of constructs for recombinant expression of full-length Sgd1 $\left(\mathrm{Sgd} 1_{\mathrm{FL}}\right)$ and the Sgd1 fragments containing the predicted structural domains MIF4G and MA3. (B) Coomassie stained SDS-PAGE gel of recombinantly expressed and purified Sgd1 full-length and Sgd1 fragments.

The interaction between Fal1 and the different Sgd1 variants was tested by immobilising MBP-tagged Sgd1 fragments on amylose beads and incubating them with recombinant $\mathrm{N}$ terminally His-tagged Fal1 (Figure 3.11).

Under the experimental conditions used, no unspecific binding of His-tagged Fal1 to the empty amylose beads (-) or beads containing the MBP-His fusion protein (MBP-His) was detected. Importantly, it was observed that full length Sgd1 (Sgd1 $\left.1_{\mathrm{FL}}\right)$ could co-precipitate His-tagged Fal1, indicating that the interaction of these proteins is direct. Interestingly, Sgd1 variants containing both the MIF4G and the MA3 domains (Sgd1 $1_{\text {MF4G-MA3 }}$ and

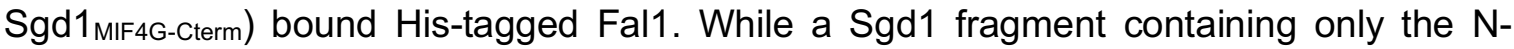
terminus and the MIF4G domain (Sgd1 $1_{\text {Nterm-MIF4G) }}$ also bound His-tagged Fal1, the construct carrying only the MA3 domain and the C-terminus of Sgd1 (Sgd1 MA3-Cterm) was $_{\text {. }}$ unable to co-precipitate the helicase. These results suggest that the MIF4G domain of Sgd1, but not its MA3 domain, is important for direct binding to Fal1. 

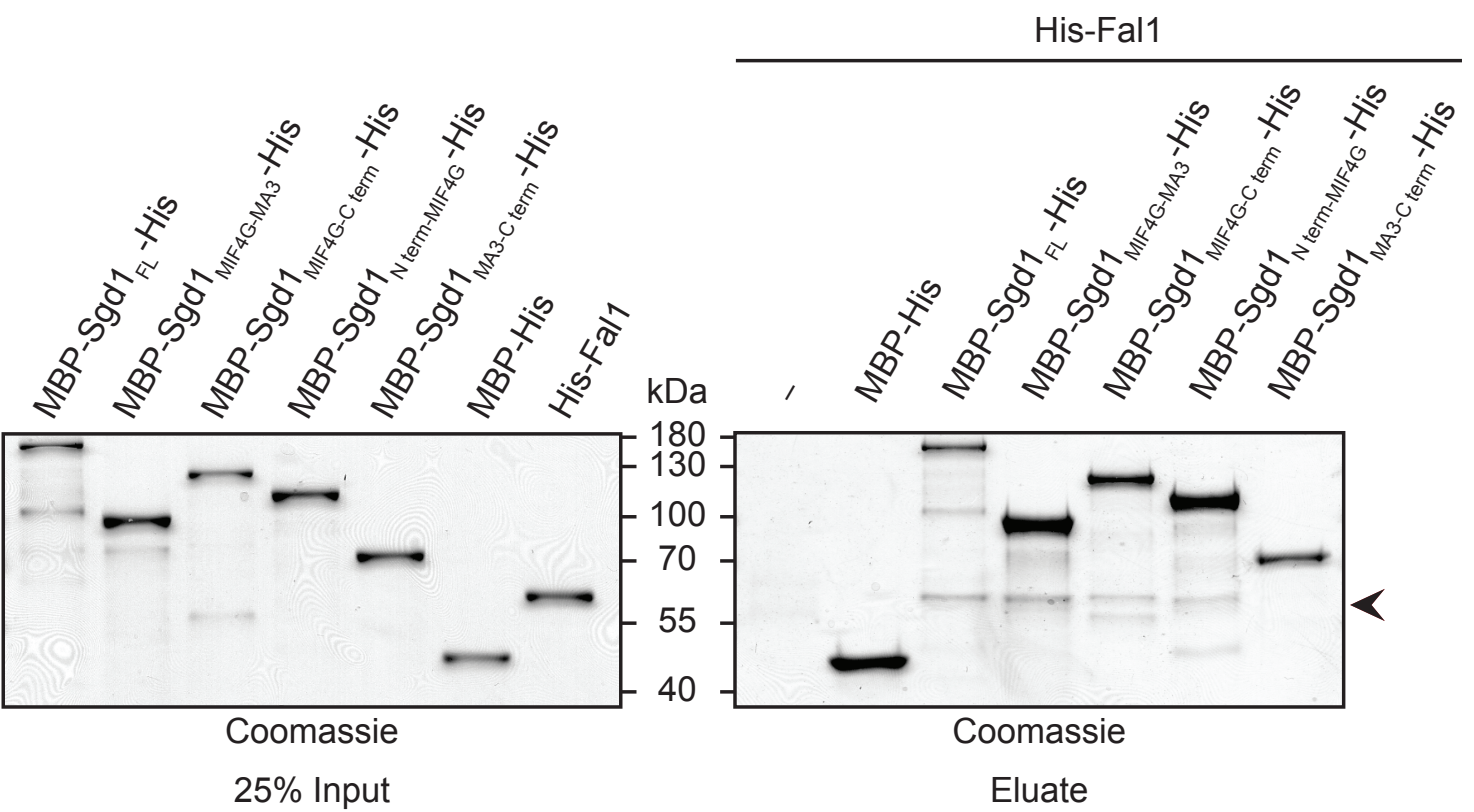

Figure 3.11 Sgd1 binds to Fal1 through its MIF4G domain. Binding assay using recombinantly expressed His-Fal1 and MBP-tagged Sgd1 fragments. MBP-tagged proteins were immobilised on amylose beads and incubated with recombinant Fal1. Proteins were visualised by Coomassie staining. Coomassie band corresponding to bound Fal1 in the elution fraction is indicated by an arrow.

To study the effect of this interaction on the ATPase activity of Fal1, in vitro NADH-coupled ATPase assays were performed. When tested for in vitro ATPase activity, recombinant full-length Sgd1 showed high non-specific ATPase activity in the presence or absence of RNA. Despite efforts to produce purer recombinant full-length Sgd1, the purification products nevertheless included various lower molecular weight contaminants. It is likely that E. coli ATP-dependent chaperones are co-purified with the recombinantly expressed Sgd1, leading to unspecific ATPase activity. As the MIF4G domain has been shown to be sufficient to alter the activity of other DEAD-box helicases (Mathys et al., 2014; Mugler et al., 2016), and the MIF4G domain of Sgd1 can directly interact with Fal1 (Figure 3.11), a His-ZZ-tagged version of the MIF4G domain of Sgd1 was recombinantly expressed and purified for use in vitro ATPase assays (Figure 3.12). 

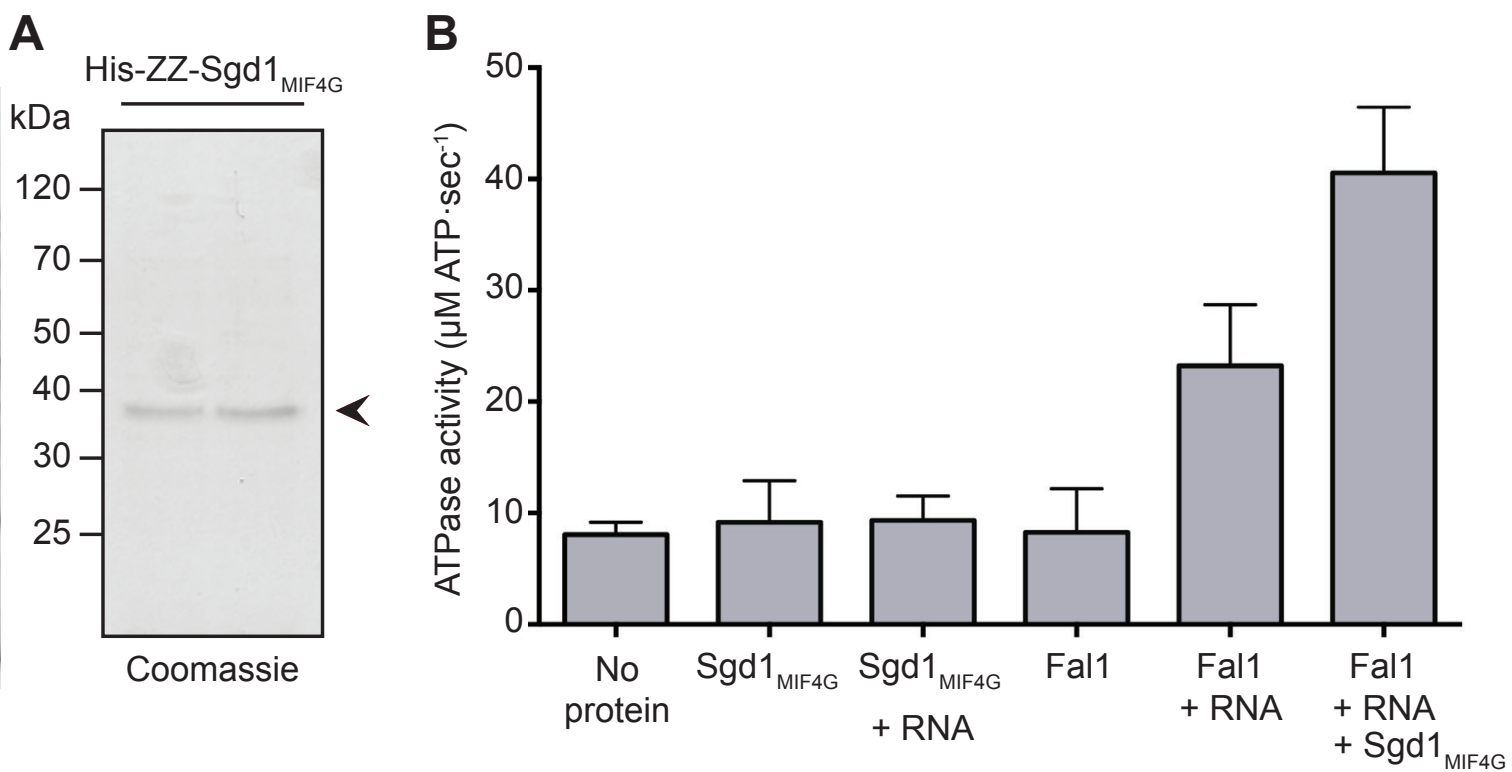

Figure 3.12 The MIF4G domain of Sgd1 can stimulate the ATPase activity of Fal1 in vitro. (A) Coomassie stained SDS-PAGE gel of recombinantly expressed and purified His-ZZ-Sgd1 MIF4G. $_{\text {. }}$ (B) NADH-coupled ATPase assay using recombinantly expressed Fal1 and MIF4G domain of Sgd1. The data from three independent experiments are presented as mean \pm standard deviation.

As expected, the purified Sgd1 MIF4G domain did not show unspecific ATPase activity beyond background, both in the absence or presence of RNA (Figure 3.12B; $\mathrm{Sgd}_{\text {MIF4G }}$ and Sgd1 ${ }_{\text {MF4G }}+$ RNA). In the absence of RNA, Fal1 did not show ATPase activity above the background ATP turnover (Figure 3.12B; Fal1), and incubation with RNA stimulated the ATPase activity of Fal1 (Figure 3.12B, Fal1 +RNA). Notably, incubation of Fal1 with RNA and the recombinant Sgd1 MIF4G showed even greater ATP hydrolysis activity (Figure 3.12B; Fal1 +RNA + Sgd1 $1_{\text {IF } 4 \mathrm{G}}$ ), suggesting that the MIF4G domain of Sgd1 can stimulate the ATPase activity of Fal1 in vitro.

\subsection{Sgd1 crosslinks to helix 12 of the $18 \mathrm{~S}$ rRNA sequence}

Identification of the binding sites of RNA-binding proteins, such as RNA helicases and others, on their substrates is key to the understanding of their biological functions. The development of crosslinking and sequencing techniques represents an important tool for achieving this. In particular, the UV-crosslinking and analysis of $\underline{c} D N A(C R A C)$ technique has allowed the identification of the binding sites of several RNA helicases implicated in ribosome biogenesis (Bohnsack et al., 2009; Brüning et al., 2018; Martin et al., 2014). 
We established that the elF4A-like helicase Fal1 is an RNA-dependent ATPase required for specific early pre-rRNA processing steps. Based on these results, it is likely that Fal1 binds a pre-rRNA substrate in vivo, and it is possible that its interacting protein, Sgd1, could induce substrate specificity by additionally binding to the corresponding RNA. To test this hypothesis, we performed CRAC experiments on yeast strains expressing HTPtagged Fal1 or Sgd1, and included a WT strain as a control. Following in culturo crosslinking and tandem affinity purification of HTP-tagged proteins under native and denaturing conditions, co-purified RNAs were radioactively labelled with ${ }^{32} \mathrm{P}$. Protein-RNA complexes were separated by electrophoresis and RNAs were detected by autoradiography (Figure 3.13).

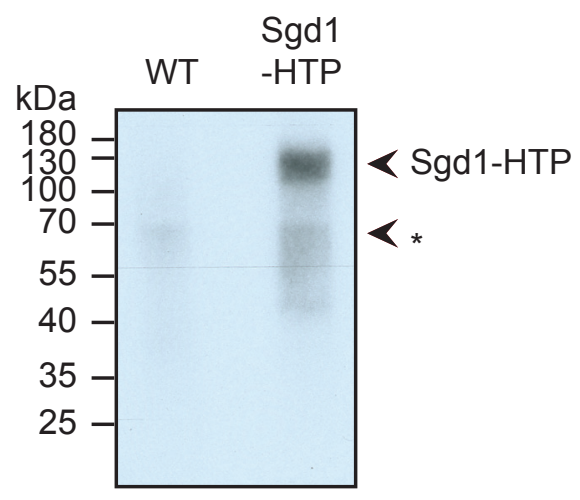

Figure 3.13 Sgd1 specifically crosslinks to RNA in vivo. Autoradiography of crosslinked proteinRNA complexes isolated from WT, HTP-Fal1 and HTP-Sgd1 strains. Arrows indicate background $\left.{ }^{*}\right)$ and complex-specific radioactive signals. Crosslinking experiments were performed by Philipp Hackert.

While the Fal1-HTP sample presented a radioactive signal at the expected size of Fal1HTP, it coincided in size with a radioactive background signal observed in the WT strain sample corresponding to RNA species non-specifically co-precipitated during the protocol. Interestingly, a radioactive signal corresponding to the Sgd1-HTP expected size was observed (Figure 3.13).

cDNA libraries were generated from the purified RNA fragments, sequenced and subjected to bioinformatic analysis as previously described (Kretschmer et al., 2018; Memet et al., 2017; Webb et al., 2014). The obtained sequencing reads were aligned to the yeast genome, and the relative proportions of reads mapping to different transcript classes were identified and compared (Figure 3.14). Interestingly, the CRAC results from the Sgd1-HTP sample (Figure 3.14A) showed enrichment of reads mapping to rDNA compared to the 
control WT strain (Figure 3.14B). This is in line with the proposed role of Sgd1 in ribosome biogenesis.

A

WT

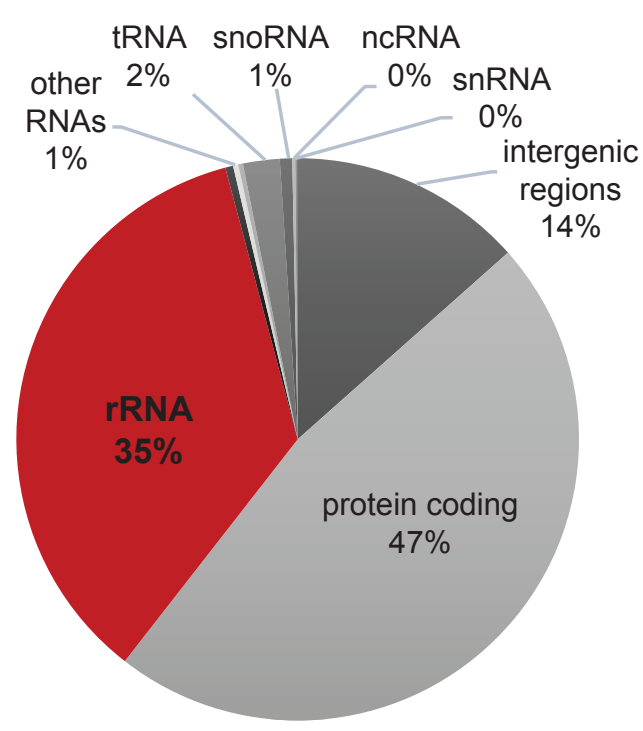

B

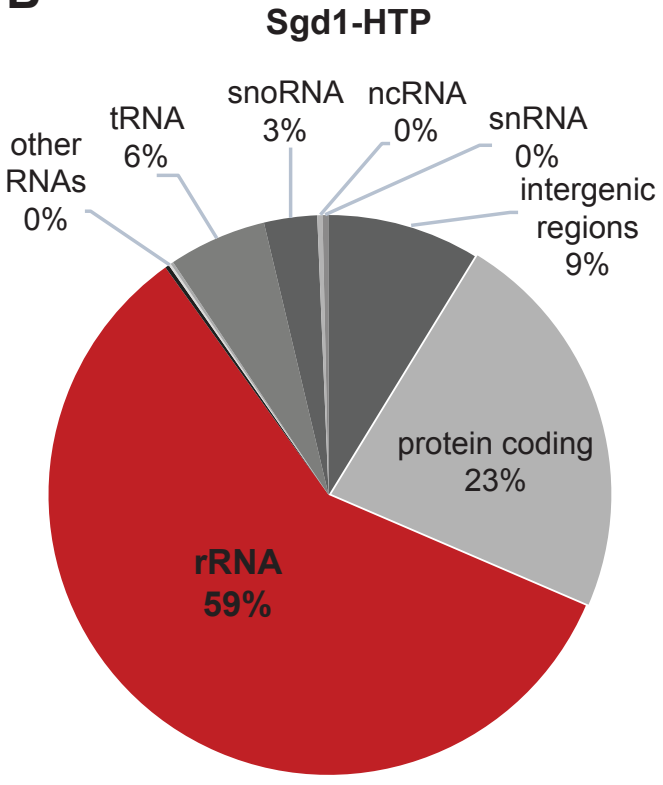

Figure 3.14 Sgd1-crosslinked RNA fragments are derived from rRNA. Wild-type (A) and Sgd1HTP (B) yeast strains were crosslinked and protein-RNA complexes affinity purified. cDNA libraries generated from the co-purified RNAs were subjected to Illumina deep sequencing and the obtained data was analysed bioinformatically. Sequencing reads were mapped to the yeast genome and the distribution of reads among the annotated different RNA species was determined. The category "other RNAs" includes pseudogenes and long non-coding RNAs. Bioinformatic analysis of the CRAC data was performed by Jens Kretschmer.

We therefore next analysed the reads that mapped to RDN37, the gene locus of a rDNA repeat, to determine the binding site of Sgd1 within the pre-rRNA sequence. To allow a direct comparison of the different CRAC experiments, the number of reads in each sample was first normalised according to the total number of mapped reads (given per million). The obtained values were plotted per nucleotide on the sequence of the $35 \mathrm{~S}$ primary transcript. At crosslinking sites, residual amino acids remain attached to the RNA after protein digestion and cause errors during the reverse transcription of the RNA (deletions or nucleotide substitutions). Therefore, mutation-aware mapping of the sequencing reads was performed and peaks of mutations within the mapped reads indicate direct contacts between proteins and RNA (Figure 3.15). 


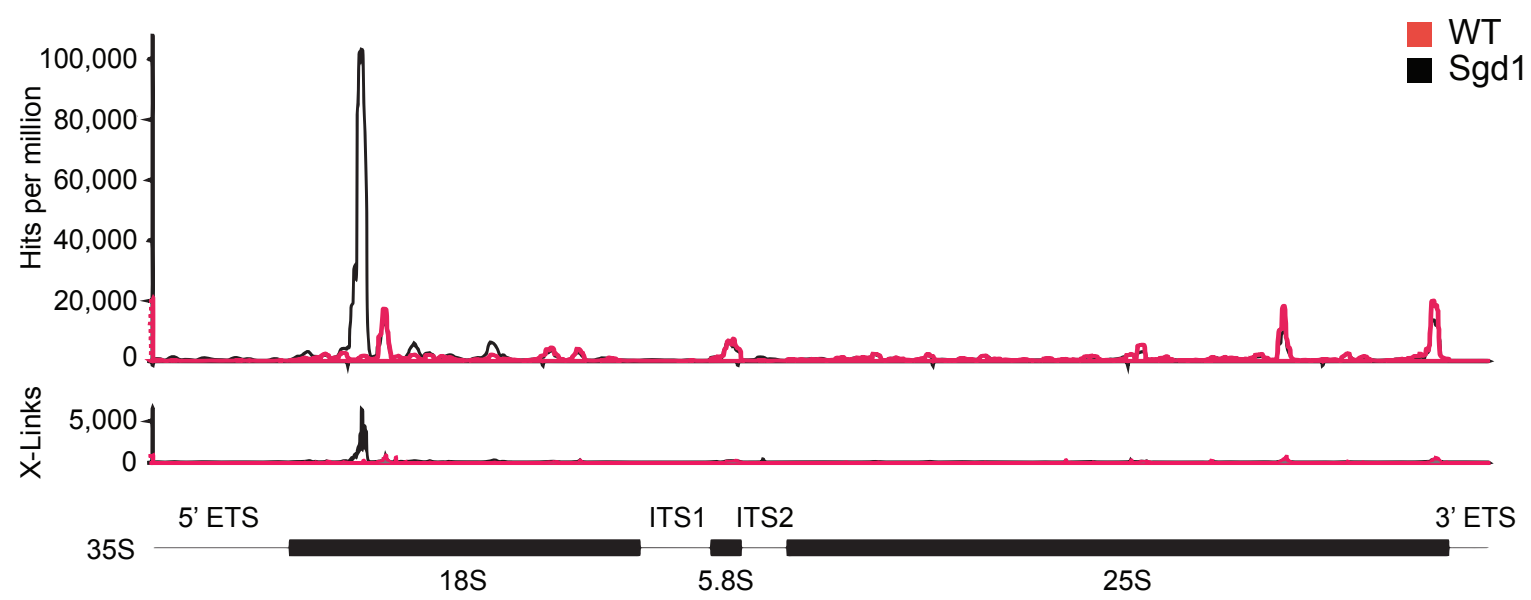

Figure 3.15 Sgd1 crosslinks within the 18S rRNA sequence of the 35S pre-rRNA. Mapping of wild-type strain (red) and Sgd1-HTP strain (black) sequencing reads to the RDN37 gene locus encoding the $35 \mathrm{~S}$ pre-rRNA transcript. The number of hits was determined from the total number of sequences overlapping with each nucleotide, normalised to the total number of mapped reads per million. Peaks of hits represent crosslinking sites at the corresponding nucleotides. Relative positions of mutations within the mapped reads derived from crosslinking are indicated (X-Links).

Interestingly, the Sgd1 CRAC sequencing reads (Figure 3.15, black) mostly mapped to a single region at the 5 ' end of the 18S rRNA sequence of the 35S pre-rRNA, which is different from the background signals detected in the CRAC sample derived from the WT strain (Figure 3.15; red). Importantly, the peak of CRAC reads overlaps with a peak of mutations within the sequenced reads (Figure 3.15; middle panel), indicating a direct contact between Sgd1 and the RNA (Figure 3.15; middle panel). These data suggest a single binding site of Sgd1 to the 18S rRNA sequence within the 35S pre-rRNA.

The normalised CRAC reads of the Sgd1-HTP sample were then mapped to the available secondary structure of the mature 18S rRNA (Petrov et al., 2014) using a colour gradient according to the number of reads mapping to each nucleotide of the $35 \mathrm{~S}$ pre-rRNA sequence (Figure 3.16). Interestingly, mapping of the CRAC reads showed that Sgd1 binds within the 5 ' domain of the 18S rRNA sequence. In particular, the Sgd1 crosslinking site overlapped with helix 12 of the mature 18S rRNA structure (Figure 3.16, left panel). 


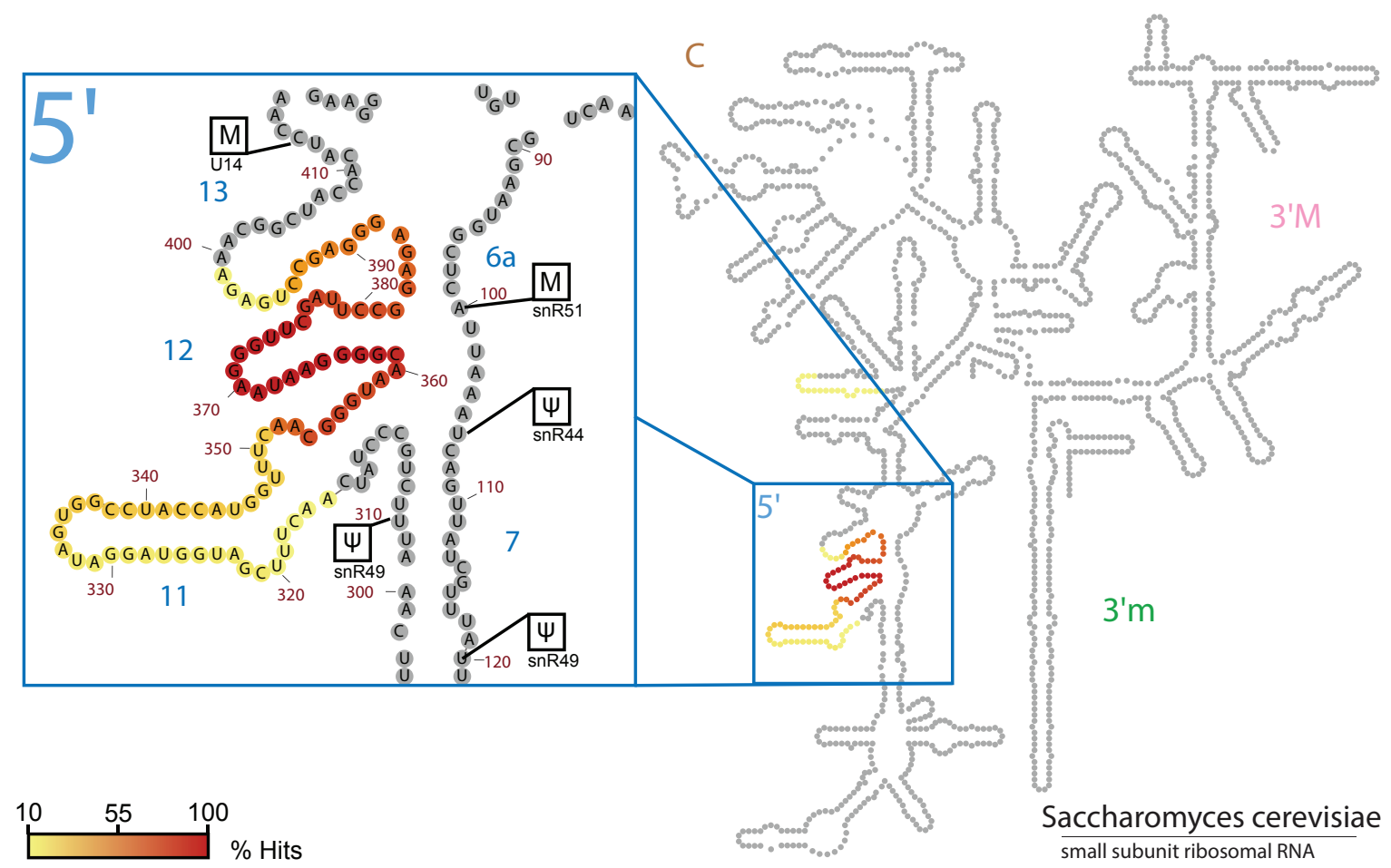

Figure 3.16 Sgd1 crosslinks to helix 12 of 18S rRNA sequence. Mapping of Sgd1 normalised CRAC reads to the secondary structure of mature 18S rRNA (Petrov et al., 2014). CRAC data is represented by colour gradient normalised to hits across RDN37 with red (100\%) corresponding to the highest peak. The left panel shows an enhance view of the binding site within the 5 ' domain of the $18 \mathrm{~S}$ rRNA sequence.

Sgd1 is required for early pre-18S rRNA processing steps, which suggests it is likely to be recruited to early pre-SSU particles. Excitingly, a structure of the earliest pre-SSU particle, the SSU processome, became recently available (Barandun et al., 2017, PDB-ID: 5WLC). Mapping the Sgd1 CRAC data to this structure can provide a better understanding of the rRNA structural context at the time of Sgd1 binding (Figure 3.17).

Interestingly, the crosslinking site of Sgd1 maps to an accessible helix within the SSU processome. Assembly factors and ribosomal proteins are organized to form a pocket around the crosslinking site of Sgd1. This could be an indication that Sgd1 is present in this early particle, but the interaction is lost during sample preparation for cryo-EM. Mass spectrometry analysis of crosslinked SSU processomes revealed an interaction between Sgd1 and Lcp5 (Barandun et al., 2017). Excitingly, Lcp5 is present in the published SSU processome structure adjacent to the Sgd1 crosslinking site identified in this study by CRAC (Barandun et al., 2017). These data support the hypothesis that Sgd1 is present in the SSU processome particle, but suggest that the interaction may be transient or the binding weak. 


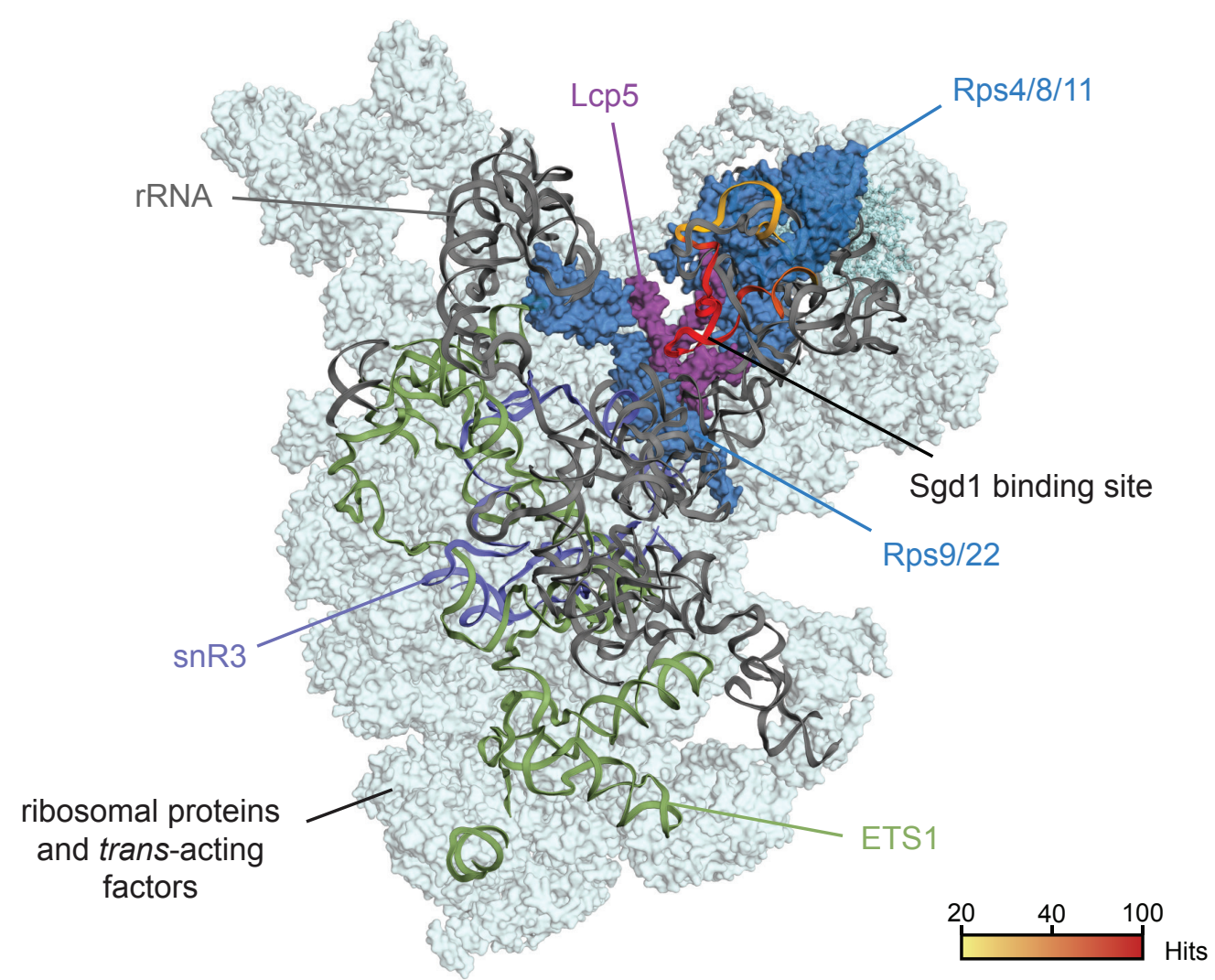

Figure 3.17 The RNA binding site of Sgd1 is located within a binding pocket in the SSU processome structure. Mapping of the Sgd1 CRAC hits onto the SSU processome particle (Barandun et al, 2017; PDB-ID: 5WLC). CRAC hits mapping follows a colour-code according to the $35 \mathrm{~S}$ sequence mapping. Densities corresponding to specific ribosomal proteins and pre-40S factors are highlighted in blue and purple. Different RNA species are indicated in colours.

\subsection{The C-terminal region of Sgd1 is responsible for RNA binding}

Our results show that Sgd1 interacts with Fal1 through its MIF4G domain, and that it also binds pre-rRNA. Interestingly, Sgd1 has not been previously reported to bind RNA or to contain any defined RNA-binding motifs. As Sgd1 contains long $\mathrm{N}$ - and C-terminal extensions flanking the central MIF4G and MA3 domains, the next aim was to determine which region(s) of Sgd1 have a role in mediating RNA binding. To address this, we engineered HTP-tagged Sgd1 truncations for complementation in yeast cells depleted of endogenous Sgd1. Upon IAA treatment, complementation strains were subjected to UVcrosslinking and pull-down of HTP-tagged proteins (test-CRAC) to test the in vivo RNAbinding ability of the N-terminus (HTP-Sgd $1_{\mathrm{N} \text {-term-MIF4G }}$ ), middle domains (HTP-Sgd $1_{\text {MIF4G- }}$

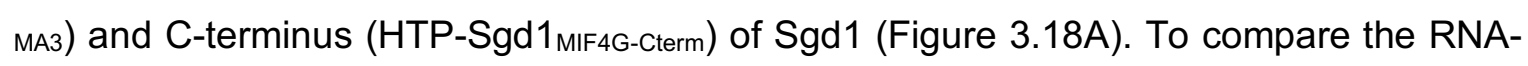
binding capacity, the expression levels of the Sgd1 fragments were normalised before denaturing PAGE of the protein-RNA complexes (Figure 3.18B). The co-purified RNAs 
were radiolabelled and protein-RNA complexes detected by autoradiography (Figure $3.18 C)$.

A

\begin{tabular}{|c|c|c|c|c|}
\hline HTP & & MIFAG & $M A 3$ & $\mathrm{HTP}-\mathrm{Sgd} 1_{\mathrm{FL}}$ \\
\hline & \multicolumn{3}{|c|}{542} & \\
\hline HTP & \multicolumn{2}{|r|}{ MIF4G } & & HTP-Sgd $1_{\text {Nterm-MIF4G }}$ \\
\hline \multicolumn{2}{|c|}{329} & 776 & & \\
\hline HTP & MIF4G & MA3 & & HTP-Sgd $1_{\text {MIF4G-MA3 }}$ \\
\hline \multicolumn{2}{|c|}{329} & \multicolumn{2}{|c|}{906} & \\
\hline HTP & MIF4G & MA3 & & HTP-Sgd $1_{\text {MIF4G-Cterm }}$ \\
\hline
\end{tabular}
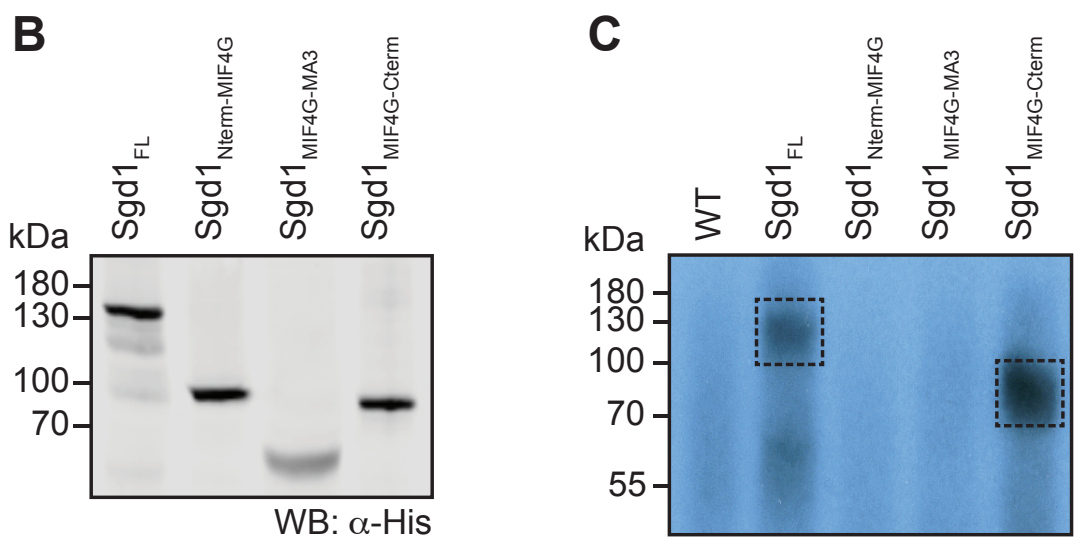

Figure 3.18 The C-terminus of Sgd1 binds RNA in vivo. (A) Schematic view of Sgd1 constructs used for in vivo test-CRAC. Constructs encoding full length (FL) Sgd1 or different regions of Sgd1 were designed for exogenous expression in yeast cells. (B) Levels of pulled-down proteins were normalised prior to analysis of protein-RNA complexes. The levels of pulled-down proteins were determined by western blotting using an antibody against the His-tag. (C) Autoradiography of radiolabelled RNA crosslinked to proteins in a wild-type strain (WT) or strains expressing different HTP-tagged Sgd1 truncations. Specific signals corresponding to RNA bound to Sgd1 $1_{\mathrm{FL}}$ and the Sgd1 C-terminus fragment are indicated with boxes.

Consistent with the previous CRAC result, we detected a radioactive signal for RNA bound

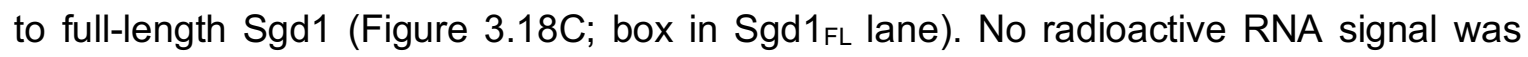
detected associated with the N-terminal fragment or the middle-domain of Sgd1

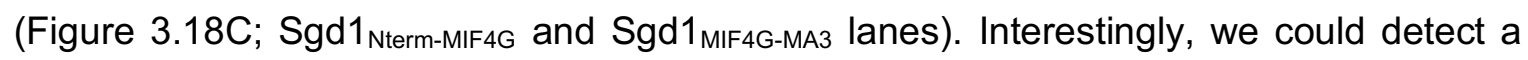
radioactive signal at a size corresponding to the C-terminal region of Sgd1 (Figure 3.18C,

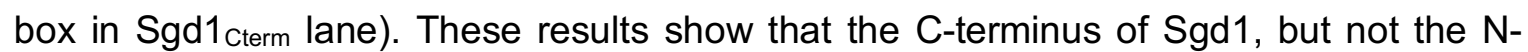
terminus or the MIF4G domain, binds RNA in vivo. 
To validate these results, we measured the in vitro RNA binding affinity of recombinantly expressed Sgd1 fragments (Figure 3.10A) by anisotropy measurements (Figure 3.19).

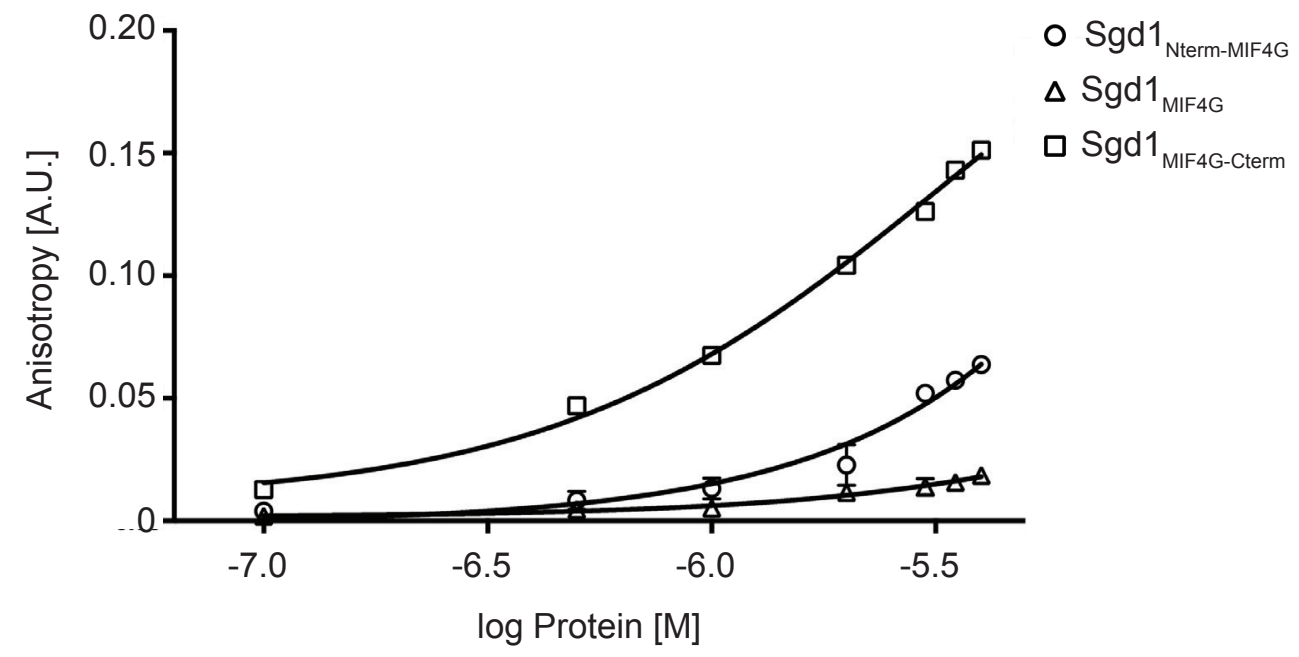

Figure 3.19 The C-terminus of Sgd1 binds RNA in vitro. Anisotropy measurements using increasing amounts of RNA and recombinant Sgd1 fragments. The data represent results from three independent experiments presented as mean \pm standard deviation.

In line with the observations from the in vivo test-CRAC experiments, a recombinant protein consisting of the N-terminal region and the MIF4G domain of Sgd1 showed little RNA binding in vitro (Figure 3.19; $\bigcirc$ ). Likewise, recombinantly expressed MIF4G domain of Sgd1 did not bind RNA in vitro (Figure 3.19; $\triangle$ ). As expected, the Sgd1 variant

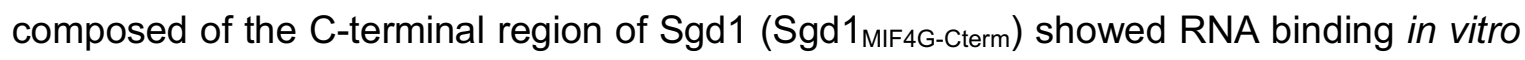
(Figure 3.19; $\square, 7.8 \pm 0.1 \mu \mathrm{M}$ ). Taken together, these results support a model where different regions of the Sgd1 sequence mediate its interaction with the RNA and Fal1. 


\section{Discussion}

\subsection{Identification of in vivo binding sites of ribosome assembly factors on pre- rRNAs}

In yeast, 21 RNA helicases are required for the biogenesis of the LSU and SSU. While these helicases have been shown to be part of different pre-ribosomal particles, a lack of information on their pre-rRNA binding sites, as well as their molecular targets within the ribosomal particles, has impeded the identification of their precise molecular functions and substrates. In this context, the aim of this study was to increase our understanding of the function of the helicases Fal1, which is involved in the maturation of the SSU. Analysis of pre-rRNA processing upon helicase depletion by northern blotting demonstrated the requirement for Fal1 for cleavage at sites $A_{0}, A_{1}$ and $A_{2}$. Interestingly, similar processing defects were observed when the MIF4G domain-containing protein Sgd1 was depleted, suggesting a shared role in the pathway. Furthermore, binding assays demonstrated that Sgd1 and Fal1 can associate both in vivo and in vitro, and the MIF4G domain of Sgd1 could stimulate the ATPase activity of Fal1 in vitro.

As Fal1 and Sgd1 are required for early cleavage steps, these proteins are expected to associate with early pre-40S ribosomal particles. However, neither Fal1 nor Sgd1 have been detected in any of the available structures of early pre-ribosomal particles. To gain insight into the function and molecular targets of these two proteins, an in vivo crosslinking technique (UV-CRAC) was utilised to identify the binding sites of Fal1 and Sgd1 on preribosomal particles. This approach allows the crosslinking of RNA-binding proteins (RBPs) to cellular RNAs by forming of a covalent link between, typically, uridine $(U)$ nucleotides in the RNA and aromatic residues of the protein (tryptophan, tyrosine, phenylalanine) (Bohnsack et al., 2012). Intriguingly, no RNA was specifically crosslinked to Fal1 under the experimental conditions employed. One possible explanation for this is that Fal1 interacts only transiently or weakly with RNA, thereby escaping the formation of a stably crosslinked complex under the aforementioned conditions. Such a transient interaction may also explain the absence of Fal1 in the recently published early pre-ribosomal particles (Barandun et al., 2017). Fal1 is a protein that belongs to the DEAD-box family of RNA helicases. These proteins are non-processive RNA helicases that bind and locally remodel their substrate RNA and then release it upon ATP hydrolysis. It is therefore possible that fast ATP turnover releases Fal1 from pre-ribosomal particles and impedes crosslinking of the protein to its pre-rRNA target. In this study, the ATPase activity of Fal1 was evaluated in vitro using recombinant proteins and a short substrate RNA. While Fal1 did not efficiently 
hydrolyse ATP under these conditions, the in vitro setup might not reflect the activity in vivo. Factors such as the real cellular substrate and the context of the pre-ribosomal particle might influence the activity of the helicase in vivo. As DEAD box helicases only dissociate from their substrate upon ATP hydrolysis, it is expected that mutants unable to hydrolyse ATP could be trapped on their cellular RNA substrates. Expression of the Fal1_DQAD mutant described in this study may therefore enable the crosslinking of Fal1 to pre-rRNAs by trapping the helicase on pre-ribosomal particles. Moreover, alternative approaches for in vivo crosslinking of proteins, such as photoactivatable ribonucleosideenhanced CRAC (PAR-CRAC), could allow an increase in crosslinking efficiency and might thereby enable mapping of interaction sites between Fal1 and pre-rRNAs. It is possible that the lack of crosslinking sites arises because the regions of the pre-rRNA that Fal1 binds to are not sensitive to the CRAC approach. As UV-CRAC preferentially crosslinks aromatic amino acids to pyrimidine nucleotides (largely uridines), if Fal1 interacts with, for example, guanosine (G)-rich regions, it would be inefficiently crosslinked. Protein-RNA contact sites that are G-rich can be identified by using the photoactivatable ribonucleoside 6-thioguanosine (6-SG), which can be incorporated into nascent RNAs and then crosslinked to proteins using light at $365 \mathrm{~nm}$. Thus, making use of the PAR-CRAC approach in combination with 6-SG might allow crosslinking of Fal1 to rRNA. Determining the pre-ribosomal binding site of Fal1 would provide insight into its recruitment to preribosomes and might allow identification of the targets of the remodelling activity as well as its function. Furthermore, analyses of additional interaction partners co-purified with Fal1 could help to identify interactors involved in ribosome biogenesis. As Fal1 is thought to establish transient interactions with rRNA and/or other proteins involved in ribosome biogenesis, to overcome this issue a formaldehyde crosslinking approach would be required. This would allow for the isolation of short-lived protein-complexes, using affinity purification methods employing TAP- or HTP-tagged Fal1.

While it was not possible to crosslink Fal1 to pre-rRNA, a binding site of Sgd1 within the 18S rRNA sequence was identified. Sgd1 was shown to directly interact with Fal1, and depletion of this protein led to pre-rRNA processing defects similar to those observed upon Fal1 depletion, suggesting a shared role in SSU maturation. Although Sgd1 has not yet been detected in any of the available cryo-EM structures of the early pre-ribosomal particles available, the pre-rRNA processing defects observed are consistent with depletion of a protein involved in maturation of the SSU processome. Furthermore, analysis of the RNAs co-purified with Sgd1 revealed an enrichment in sequencing reads mapped to the U3 snoRNA, a core component of the SSU processome, strongly supporting the hypothesis that Sgd1 is present in the SSU processome. Mapping of the Sgd1 CRAC 
data to the latest cryo-EM structure of the SSU processome particle (Barandun et al., 2017; PDB-ID: 5WLC) revealed a binding site of Sgd1 to an accessible helix located in the 5 ' domain of the 18S rRNA sequence. Interestingly, assembly factors and ribosomal proteins present in the structure form a cleft around the area identified as binding site of Sgd1. The protein could therefore interact with residues positioned in this region. Furthermore, the identified Sgd1 binding site is adjacent to that of the assembly factor Lcp5. Interaction studies by yeast two-hybrid experiments and mass spectrometry analyses of protein-protein crosslinked SSU processomes have previously reported an interaction between Lcp5 and Sgd1 (Barandun et al., 2017; Vincent et al., 2018). While these data strongly support that Sgd1 is a component of the SSU processome, it is likely that the interaction between Sgd1 and pre-ribosomal particles is relatively weak and Sgd1 is often lost during the purification of pre-ribosomes. Mass spectrometry analyses of proteins co-purified with Sgd1 itself might reveal other interaction partners within the preribosomal particle. Broader knowledge of the interaction network of Sgd1 within the SSU processome and structural data on Sgd1, would complement the CRAC results and allow for a precise placement of the protein onto the available structure

While no defined RNA binding domains are present in Sgd1, this study demonstrates that the C-terminal region of this protein can be crosslinked to RNA in vivo and also binds RNA in a non-sequence specific manner in vitro. Moreover, this relatively large protein was shown to interact with Fal1 through its MIF4G domain forming a complex. Although Fal1 could not be crosslinked to rRNA, depletion and complementation assays showed that the ATPase activity of the helicase is required for early processing events in 18S rRNA maturation. These data, together with the CRAC results for Sgd1, suggest a model in which Sgd1 stably binds to the pre-rRNA via its C-terminus and simultaneously interacts with Fal1 via its MIF4G domain, whereupon the ATPase activity of Fal1 is stimulated to enable the helicase to locally remodel a particular pre-RNA substrate. Importantly, it remains unclear whether formation of the Sgd1-Fal1 complex is required for recruitment of those proteins to pre-ribosomes. Related RNA helicase-cofactor pairs are recruited to their cellular targets as a complex, such as elF4A and elF4G (Jackson et al., 2010). In contrast, the human RNA helicase elF4AIII can be recruited to spliced pre-mRNAs in complex with CWC22 or individually by interacting with RNA-bound CWC22 (Steckelberg et al., 2015). As Fal1 is only poorly associated with pre-ribosomal particles, determining whether Sgd1 is required for the recruitment of the helicase to pre-ribosomes will prove to be challenging. Alternatively, by disrupting interactions between Fal1 and Sgd1 in vivo it would be possible to study whether complex formation is required for the functions of these proteins. Interestingly, a genetic screen identified residues in Fal1 and Sgd1 important for the 
interactions between both proteins (Alexandrov et al., 2011). Generation of interaction mutants by amino acid substitution would therefore allow us to study the recruitment of Fal1 and Sgd1 to pre-ribosomal particles independently of complex formation.

\subsection{Molecular function of RNA helicases in ribosome biogenesis}

RNA helicases are a ubiquitous class of proteins that contain a structurally conserved helicase core and are primarily anticipated to act as unwinders of RNA duplexes. However, their reported molecular functions have been expanded to include annealing of RNA strands and acting as RNA clamps within RNP complexes. This broad repertoire of molecular functions places RNA helicases as key players in many RNA metabolism pathways such as transcription, pre-mRNA splicing and ribosome biogenesis. For many of the 21 RNA helicases involved in ribosome biogenesis, combinations of genetic and biochemical studies have linked them to the release and/or recruitment of snoRNPs or other modification enzymes to the pre-rRNA, displacement or recruitment of proteins from pre-ribosomal particles or structural remodelling of pre-rRNA regions (Brüning et al., 2018; Khoshnevis et al., 2016; Martin et al., 2013; Sardana et al., 2015; Sharma et al., 2017; Soltanieh et al., 2015). However, it remains mostly unclear whether these functions represent direct or indirect effects of the helicase action.

During early steps of ribosome biogenesis, snoRNAs guide pre-rRNA modification or influence pre-rRNA folding by forming stable base-pairing interactions with pre-rRNA sequences. The dissociation of some snoRNPs from pre-ribosomes may therefore require the action of proteins, and the unwinding activity of RNA helicases places them as good candidates for mediating release of snoRNAs. Interestingly, the requirement of RNA helicases for release of particular snoRNAs from pre-ribosomes likely involves both direct and indirect mechanisms. A clear example of this involves the RNA helicases Has1 and Dbp4; accumulation of the snoRNA U14 on pre-ribosomal particles was reported upon depletion of Has1 as well as Dbp4 (Koš and Tollervey, 2005; Liang and Fournier, 2006). However, recently binding sites of Has1 on pre-rRNA and on the U14 snoRNA were identified demonstrating that the helicase binds to the interacting regions of both RNAs. These results, together with the detection of chimeric rRNA-snoRNA sequences bound by Has1 in vivo, strongly support a direct role of Has1 in destabilising U14-pre-18S rRNA base pairing (Brüning et al., 2018). In contrast, Dbp4 does not associate with the U14 snoRNA, suggesting that the requirement for Dbp4 for release of U14 occurs by an indirect mechanism reflecting the presence of both these components in early pre-ribosomal particles (Soltanieh et al., 2015). Interestingly, a distinction in the mode of action of 
processive and non-processive RNA helicases suggests that these two classes of proteins perform similar functions by different mechanisms. For example, the DEAH-box helicases Prp43 and Dhr1 are proposed to directly unwind snoRNAs from pre-ribosomal particles (Bohnsack et al., 2009; Sardana et al., 2015). RNA helicases belonging to the DEAH-box family are processive translocases and likely achieve unwinding of snoRNAs by translocating along the pre-rRNA. Alternatively, DEAD-box proteins such as Has1 are nonprocessive helicases and unwind local RNA duplexes, thus suggesting that Has1 may instead achieve release of snoRNPs by (partially) destabilising snoRNA-pre-rRNA base pairing.

Some RNA helicases are proposed to regulate other aspects of pre-ribosomal RNPs dynamics, such as structural rearrangements, incorporation of ribosomal proteins and release or recruitment of AFs. As for snoRNA release, different mechanisms are proposed for pre-rRNA remodelling and protein displacement. As processive translocases, DEAHbox helicases are predicted to unwind longer RNA duplexes and be able to displace proteins with a larger 'footprint' (Jankowsky, 2001). For example, the DEAH-box helicase Prp43 remodels the 3' end of the 18S rRNA precursor, a key step for D site cleavage by Nob1 in the cytoplasm. It is unclear, though, whether this function consists of direct pre-rRNA remodelling or if it includes protein displacement (Bohnsack et al., 2009; Pertschy et al., 2009). Also, Dhr2 is an uncharacterised DEAH-box protein that does not seem to have a role in modulating the dynamics of snoRNAs on pre-ribosomes (Bohnsack et al., 2008). Instead, it might be required for structural remodelling and/or protein displacement. The only RNA helicase which does not belong to SF2, the Ski2-like helicase Mtr4, is a processive translocase. In line with the proposed roles for the DEAH-box translocases, the unwinding activity of Mtr4 is required for the remodelling of large rRNA structures and/or protein displacement to facilitate delivery of the pre-rRNA processing intermediate to the nuclear exosome (Johnson and Jackson, 2013). Many DEAD-box helicases have been proposed to introduce structural rearrangements of rRNPs and modulate the release and incorporation of proteins. For instance, the ATPase activity of the DEAD-box helicase Rok1 has been proposed to govern the release of AF Rrp5 from pre-40S particles. The same study reports that Rok1 can directly release Rrp5 from preribosomal particles by inducing conformational changes upon ATP hydrolysis (Khoshnevis et al., 2016). Also, RNA helicases have been proposed to assist in establishing rRNA-folds necessary for the recruitment of ribosomal proteins. For example, Has1 is suggested to coordinate conformational changes within domain I of the $5.8 \mathrm{~S} / 25 \mathrm{~S}$ pre-rRNA that drive stable incorporation of several ribosomal proteins. While the recently available cryo-EM structure of a pre-60S particle and the CRAC data support a role in domain I formation, 
the exact function of Has1 remains unknown (Brüning et al., 2018; Dembowski et al., 2013; Zhou et al., 2019). Interestingly, non-processive DEAD-box helicases unwind short RNA duplexes locally and would therefore be unable to directly remove proteins (Chen et al., 2008). Protein removal could then be achieved indirectly by remodelling of structures proximal to the displaced protein. It is thereby possible that the inability of DEAD-box helicases to remove a set of proteins might control their unwinding activity and prevent RNP disassembly.

Phenotypical classification in a Fal1 depletion strain placed Fal1 as an RNA helicase necessary for early pre-rRNA cleavages that are required to generate the $18 \mathrm{~S}$ rRNA. While impaired processing at sites $A_{0}, A_{1}$ and $A_{2}$ has been associated with impaired release of the U3 snoRNA, a previous study showed that depletion of Fal1 does not affect association or release of U3 or any other snoRNAs from pre-ribosomal particles (Bohnsack et al., 2008; Sardana et al., 2015). Interestingly, it was proposed that RNA helicases may have redundant roles in modulating the dynamics of snoRNAs, and depletion of a single helicase may not show significant changes in snoRNA association (Bohnsack et al., 2008). However, Fal1 is essential for cell viability (Kressler et al., 1997), implying that, although a redundant function in modulating snoRNA dynamics cannot be disregarded, it is likely that Fal1 has an alternative role during ribosome assembly.

In order to elucidate the molecular function of Fal1, it was attempted to identify the binding site(s) of the helicase on the rRNA precursors. While identification of the pre-rRNA crosslinking sites of RNA helicases can provide a basis for determining their functions in the ribosome assembly pathway, it is important that a distinction is made between binding sites contacted by the helicase core, hence "remodelling sites", and binding sites that reflect docking positions of the helicase onto the target RNP. Interestingly, Fal1 is a socalled "minimal" DEAD-box helicase, which does not have $\mathrm{N}$ - and C-terminal extensions flanking the helicase core (Andreou and Klostermeier, 2013). It is therefore expected that Fal1 establishes interactions with the rRNA only via the RNA binding motifs present in the helicase core. Other RNA helicases contain N- and C-terminal extensions that can often also mediate interactions with RNA and tether the helicase core in proximity to a substrate helix. For instance, a recent study identified two distinct binding sites of the helicase Has1 in the 18S rRNA sequence. Analyses of the CRAC data allowed identification of one of the binding sites as an active target site for snoRNA release, while the second binding site was proposed to represent a binding platform for the helicase. Although both binding sites are found in distant region in mature $40 \mathrm{~S}$ particles, it is proposed that they might be in close proximity in Has1-containing pre-ribosomal particles (Brüning et al., 2018). This concept is in line with the described mechanisms of other RNA helicases acting on RNP 
complexes. For example, the basic C-terminal tail of the DEAD-box helicase Mss116 is proposed to fix the helicase to RNPs, allowing the helicase core to interact with and remodel proximal RNAs which may only be close in a tertiary conformation (Mallam et al., 2011). Interestingly, the cryo-EM structure of Has1 bound to an early pre-LSU particle showed an additional binding site. While the rRNA is contacted by both RecA-like domains, these interactions and the conformation of the helicase core domains are different than those observed in a closed active conformation, suggesting that this binding site represents a docking site of the helicase rather than a remodelling site (Zhou et al., 2019).

Another important aspect for understanding the functional roles of RNA helicases in ribosome biogenesis is the identification of the pre-ribosomal particle(s) they associate with. A comprehensive view of the pre-ribosomal particles that either Fal1 or Sgd1 associate with has remained elusive. While the data suggest that these proteins operate as components of the SSU processome (discussed in section 4.1), no information is available on the temporal order of events, from recruitment of the helicase and its cofactor to the pre-ribosomal particles to their release. Studying the presence of Fal1 and/or Sgd1 in isolated pre-ribosomal particles corresponding to different stages of early $40 \mathrm{~S}$ maturation would help to identify the time of association and release of these factors. A time course of $40 S$ maturation can be established by purifying stage-specific particles via tagged biogenesis factors whose duration of association is already known (Barandun et al., 2018). For this approach, protein-protein crosslinking would likely be required to stabilise the transient interactions of Fal1 and Sgd1 with pre-ribosomal particles. Importantly, recruitment of a helicase to a specific pre-ribosomal particle may be uncoupled from its remodelling function, which could take place at a later stage. This can be due to stage-specific activation of the helicase and/or inaccessibility of the substrate. This concept is well exemplified by another assembly factor, the endonuclease Nob1, which is responsible for D site cleavage of the 3' end of the 18S rRNA sequence (Pertschy et al., 2009). While Nob1 associates with nuclear pre-40S particles, $D$ site cleavage is impaired by substrate inaccessibility. The endonuclease thus remains inactively associated, and cleavage competence is achieved only in the cytoplasm once the cleavage site on the pre-18S rRNA substrate becomes accessible (Ameismeier et al., 2018). This illustrates how identification of the binding sites of RNA helicases on the pre-rRNA, as well as their molecular targets in the pathway, is essential to achieve a comprehensive understanding of the molecular function of these enzymes. Combination of CRAC data and the identification of Fal1-associated pre-ribosomal particle(s) will facilitate functional insight into the role of the helicase at its binding site(s). For example, in vivo structure probing experiments can be employed to identify rRNA rearrangements in isolated pre-ribosomal 
particles containing or depleted of Fal1. This method relies on differential nucleotide accessibility to a modifying agent, such as dimethyl sulphate (DMS) upon depletion of the helicase. Treatment of RNA with DMS induces $N^{3}$ - and $N^{1}$-methylation of non-base-paired and non-protein-bound ("accessible") cytidine and adenosine residues respectively. Changes in nucleotide accessibility in regions proximal to the helicase binding site can then be monitored by primer extension, as modified residues hinder progress of a reverse transcriptase (Swiatkowska et al., 2012). Alternatively, mass spectrometry analysis of preribosomal particles isolated in the presence or absence of Fal1 could reveal functions in modulating the composition of pre-ribosomes, i.e. protein recruitment or release.

\subsection{Alternative pre-rRNA processing pathways}

Ribosome biogenesis is one of the most energy consuming processes in the cell. Up to $70 \%$ of all transcription is directed to ribosome biogenesis. As such, it is tightly regulated by environmental changes and nutrient availability (Warner, 1999), and thus the TORC1 pathway has emerged as an important regulator of ribosome production. Upon environmental stresses such as heat/cold shock, low $\mathrm{pH}$, or nutrient starvation, downstream effectors of the TORC1 pathway regulate multiple aspects of ribosome production, such as transcription of the rDNA locus as well as transcription of genes encoding ribosomal proteins and genes encoding ribosome assembly factors (reviewed in de la Cruz et al., 2018).

Recent studies reported that changes in environmental conditions, such as nutrient starvation or stress, produce a switch in yeast cells between two alternative pre-rRNA processes (Kos-Braun et al., 2017; Talkish et al., 2016). In exponentially growing yeast, most pre-rRNA processing follows the so-called $\mathrm{A}_{2}$ pathway, named after the cleavage site that separates the $25 S$ and $18 \mathrm{~S}$ maturation pathways. Cleavage of the $35 \mathrm{~S}$ pre-rRNA at site $A_{2}$ within the ITS1 generates the $20 S$ and $27 S A_{2}$ pre-rRNAs, which can be rapidly processed into the 18S, $25 \mathrm{~S}$ and 5.8S mature rRNAs (Fernández-Pevida et al., 2015). However, under diverse stress conditions there is a switch of pre-rRNA processing from an $A_{2}$ to an $A_{3}$ pathway in which the $35 S$ pre-rRNA is cleaved at site $A_{3}$, leading to $23 \mathrm{~S}$ and $27 \mathrm{SA}_{3}$ pre-rRNAs. Strikingly, pulse-chase experiments demonstrated that under these conditions, rDNA transcription is decreased and the production of mature ribosomes is stalled. Thus, switching to the $A_{3}$ pathway leads to non-productive pre-rRNA processing, as the $23 \mathrm{~S}$ and $27 \mathrm{SA}_{3}$ pre-rRNAs cannot be further processed into mature rRNAs. The TORC1 pathway has been shown to control the switch between the $A_{2}$ and $A_{3}$ 
pathways by a mechanism that involves casein kinase 2 (CK2) and coordinates the arrest of the production of both ribosomal subunits (Kos-Braun et al., 2017).

Production of the 23S pre-rRNA was reported more than two decades ago (Dunbar 1997), but the physiological relevance of $23 S$ production remained mostly unknown. The recently discovered link between $23 \mathrm{~S}$ accumulation and TORC1 inactivation upon stress attributed a physiological role to 235 production when environmental stimuli repress ribosome production (Kos-Braun et al., 2017; Talkish et al., 2016). Under stress conditions, reduced rDNA transcription and non-productive ribosome biogenesis allow the pathway to work at a minimum level of energy requirement without completely suppressing it. While switching to a non-productive processing might seem an ineffective use of energy, this strategy allows a rapid response to favourable environmental conditions. In contrast, complete arrest of rDNA transcription would lead to nucleolar dissolution and diffusion of ribosomal factors (Turner et al., 2012), thus delaying restart of ribosome production once environmental conditions become favourable again. Continuous minimal production of rRNA allows yeast cells to rapidly respond to nutrient-rich (favourable) conditions, providing an evolutionary advantage to rapidly resume growth and outcompete other organisms.

Interestingly, production of a dead-end 23S precursor is also observed upon depletion or mutation of early AFs and RPs in exponentially growing cells (Henras et al., 2015). However, in contrast to the stress response, aberrant processing in depletion strains only affects $18 \mathrm{~S}$ rRNA production and not 25S rRNA. While 23S pre-rRNA cannot be further processed, $27 \mathrm{SA}_{3}$ is matured into $25 \mathrm{~S}$ and $5.8 \mathrm{~S}$ rRNA, leading to an imbalance in the levels of different ribosomal subunits (Kos-Braun et al., 2017). These defects reflect different physiological roles of the $23 \mathrm{~S}$ pre-rRNA produced upon impaired ribosome biogenesis and in stress conditions. Nonetheless, stress-related accumulation of $23 \mathrm{~S}$ pre-rRNA in depletion strains can lead to mis-interpretations of depletion results. Most depletion systems (e.g. pGAL, pTet) require long periods of growth in non-permissive conditions (e.g. 12 h). Under these conditions yeast cells, can enter post-diauxic shift, resulting in stress-related $23 \mathrm{~S}$ production and indirect pre-rRNA processing defects. Moreover, long-term depletion of essential proteins subjects the cells to immense levels of stress. Thus, the pre-rRNA processing defects arising when working with long depletion times must be carefully evaluated. Importantly, the auxin degron system used in this study allowed fast depletion of endogenous essential proteins within 60 min of auxin treatment, reducing the level of stress cells are exposed to. Furthermore, depletion of Fal1 and Sgd1 leads to specific reduction of $18 \mathrm{~S}$ rRNA precursors, but not of late $25 S$ rRNA precursors. 
Therefore, rather than an indirect effect of stress, the 23S accumulation observed upon depletion reflects the requirement for Fal1/Sgd1 for $A_{2}$ cleavage.

\subsection{Recruitment and regulation of RNA helicases}

RNA helicases have important and functionally distinct roles in many different cellular processes. Paradoxically, these proteins often lack sequence specificity for their substrate RNAs as, for instance, the RNA binding motifs present in the helicase core establish interactions mainly with the phosphate backbone of the RNA. Considering these characteristics, the question arises of how these enzymes are specifically recruited to their cellular substrates? Some RNA helicases carry additional RNA binding domains (RBD) that bind specific sequences or secondary structures in their target RNAs, as is the case of the RBD in the C-terminal extension of the $E$. coli helicase DbpA that recognises and binds a structure in the 23S rRNA (Rudolph and Klostermeier, 2015). Other RNA helicases can be recruited to their RNA substrates in a non-sequence-specific manner by ancillary domains, like the basic C-terminal tail in the DEAD-box helicase Mss116 that tethers the helicase to unstructured RNAs (Mallam et al., 2011). In contrast, many RNA helicases establish protein-protein interactions with cofactor proteins that target them to their specific RNA substrates. Moreover, the intrinsically autoinhibitory conformation of some RNA helicases prevents the unspecific action of these enzymes on non-substrate RNAs, and in this context cofactor proteins can provide an additional layer of regulation by only inducing the activity of a cognate helicase at a specific time or in a particular context (Sloan and Bohnsack, 2018).

In recent years, families of cofactor proteins with common features have emerged as regulators of particular types of RNA helicases. A well characterised family of cofactors are the G-patch proteins, named after a glycine-rich region (G-patch) that mediates the interaction with the RNA helicase. These proteins interact with DEAH-box RNA helicases and stimulate their activity by a poorly understood mechanism (Robert-Paganin et al., 2017; Tauchert et al., 2017). Another important group of RNA helicase interactors are the MIF4G domain-containing proteins. These proteins carry the middle domain of the translation initiation factor elF4G, consistent of five HEAT repeats (MIF4G domain), which mediates the binding to elF4A-like DEAD-box helicases. Strikingly, and despite the similarity of the MIF4G domains of different proteins, structural analyses of several cofactor-helicase complexes showed that MIF4G domain-containing proteins can either positively or negatively affect the catalytic activity of DEAD-box helicases. For example, the activity of elF4A is stimulated upon conformational changes induced by elF4G binding resulting in a highly active conformational state (Hilbert et al., 2011; Schutz et al., 2008). 
In contrast, binding of the MIF4G domain-containing protein CWC22 to elF4AIII leads to formation of an inactive conformation of the helicase (Buchwald et al., 2013; Steckelberg et al., 2012).

This thesis investigates the potential role of the MIF4G domain-containing protein Sgd1 as a cofactor of the DEAD-box helicase Fal1. A direct interaction between Fal1 and Sgd1 was demonstrated by in vitro binding assays, and it was shown that these two proteins associate in vivo in an RNA-independent manner. Furthermore, it was determined that the interaction between Fal1 and Sgd1 is mediated by the MIF4G domain of the cofactor, supporting the hypothesis that these two proteins form an elF4A-elF4G like complex. These data, together with the similar pre-rRNA processing defects observed upon depletion of either protein, support a role of a Fal1-Sgd1 complex in ribosome biogenesis. The requirement for complex formation for $18 \mathrm{~S}$ rRNA production could be studied using Fal1 or Sgd1 mutants that abolish the interaction and thus complex formation, as proposed in section 4.1 .

Interestingly, our data suggest that the MIF4G domain of Sgd1 can stimulate the ATPase activity of the helicase in vitro. While the stimulation observed in vitro by the MIF4G domain was not strong, it is likely that other elements of Sgd1 beyond the MIF4G domain contribute to the stimulation of Fal1 in the cell. It is expected that full-length Sgd1 or longer variants of Sgd1 would further stimulate the ATPase activity of Fal1 in vitro. As Fal1 and Sgd1 display characteristics of an elF4A-eIF4G like complex, and due to the lack of structural information of Fal1 or Sgd1, our understanding of the molecular mechanism of helicase activation is limited to the available structures of the elF4A-elF4G complex (Schutz et al., 2008). However, despite the structural conservation of the MIF4G domain, previous biochemical analyses showed that different MIF4G domains specifically bind and modulate the activity of their cognate helicases, but not other DEAD-box helicases. This suggests that so far unidentified elements either within the MIF4G domains or within the helicases endow interaction specificity enabling only specific complexes to be formed (Mugler et al., 2016). Structural analyses of additional RNA helicase-cofactor complexes will allow for a better understanding of how the MIF4G domain and/or other domains interact with RNA helicases to achieve such specificity. In particular, Sgd1 is a relatively large protein carrying long $\mathrm{N}$ - and $\mathrm{C}$-terminal extensions that flank the conserved MIF4G domain. Interactions between MIF4G domain-containing proteins and RNA helicases are suggested to be primarily established through the MIF4G domain. Structural information of the Fal1-Sgd1 complex could shed light on how these additional regions of Sgd1 may affect the interaction with Fal1 and the overall architecture of the Fal1-Sgd1 complex. Furthermore, swapping the MIF4G-domain of Sgd1 for those of other MIF4G-containing 
proteins, such as Gle1, could prove useful for understanding the specificity of Sgd1 for Fal1 and the resulting stimulation of helicase activity, in vitro and potentially in vivo. Such an approach has been successfully used for analysing the specificity of G-patch domains for interactions with their cognate RNA helicases (Banerjee et al., 2015; Fourmann et al., 2017).

Interestingly, the human homolog of Fal1 (elF4AIII) is a multifunctional helicase, and thereby a member of a growing class of RNA helicases reported to have roles in more than one cellular pathway. Families of cofactors play important roles in regulating the activity and localisation of such multifunctional helicases, as one multifunctional helicase may interact with several related cofactors that target it to different cellular functions. Human elF4AIII has two MIF4G domain-containing interaction partners that act together with the helicase in two central aspects of RNA metabolism; elF4AIII interacts with NOM1 in ribosome biogenesis, and in exon-junction complex (EJC) assembly, elF4AIII interacts with CWC22 (Alexandrov et al., 2011; Steckelberg et al., 2015). Strikingly, binding to the different MIF4G domain-containing proteins not only recruits the helicase to different pathways, but also changes the molecular function of the helicase between an active ATPase in ribosome biogenesis and an RNA clamp in EJC assembly (Buchwald et al., 2013; Steckelberg et al., 2015). As elF4AIII and the MIF4G domain-containing proteins interact through the MIF4G domain, this raises the possibility that the cofactor proteins compete for binding to the RNA helicase. Competitive interaction of cofactors with an RNA helicase has been demonstrated for the multifunctional helicase Prp43 in yeast. This helicase is required for pre-mRNA splicing and ribosome biogenesis, and can interact with four G-patch proteins which can each stimulate its catalytic activity. A recent study showed that these G-patch proteins bind to Prp43 in a mutually exclusive manner. Furthermore, this study demonstrated that the balance between cofactor proteins regulates the subcellular distribution of the RNA helicase between pathways (Heininger et al., 2016). Thus, the yeast G-patch proteins form a network of cofactors that can mediate crosstalk between the different cellular pathways in which Prp43 is required. Interestingly, the number of G-patch proteins and MIF4G domain-containing proteins increases from yeast to humans (five G-patch proteins and seven MIF4G domain-containing proteins identified in yeast, 12 MIF4G domain-containing proteins and 22 G-patch proteins in humans). This likely reflects a relative increase in the complexity of human gene expression and therefore the need for a more extensive network of potential regulators. Importantly, these examples illustrate how interactions with a network of cofactor proteins provides an elegant mechanism for the spatial and temporal regulation of multifunctional RNA helicases that, otherwise, lack substrate specificity (Sloan and Bohnsack, 2018). 
So far, Sgd1 is the only reported direct interaction partner of Fal1, and in contrast to human elF4AIII, no functions have been ascribed to the helicase outside ribosome biogenesis. Interestingly, yeast cells express a homologue of CWC22, yeast Cwc22, which also carries MIF4G and MA3 domains. Cwc22 is involved in pre-mRNA splicing, and is necessary for the role of Prp2 in promoting the release of the $U 2$ components (Yeh et al., 2011). As yeast cells lack other EJC components, such as Y14 and Mago, and an interaction between Cwc22 and Fal1 has not been reported yet, it remains unknown if yeast Fal1 also operates as a multifunctional protein, or whether extra functions have only been evolved in higher eukaryotes. 


\section{References}

Abdelhaleem, M. (2004). Do human RNA helicases have a role in cancer? Biochim. Biophys. Acta 1704, 37-46.

Aitken, C.E., and Lorsch, J.R. (2012). A mechanistic overview of translation initiation in eukaryotes. Nat. Struct. Mol. Biol. 19, 568-576.

Akhtar, N., Påhlman, A.-K., Larsson, K., Corbett, A.H., and Adler, L. (2000). SGD1 encodes an essential nuclear protein of Saccharomyces cerevisiae that affects expression of the GPD1 gene for glycerol 3-phosphate dehydrogenase. FEBS Lett. 483, 87-92.

Alexander, R.D., Barrass, J.D., Dichtl, B., Kos, M., Obtulowicz, T., Robert, M.-C., Koper, M., Karkusiewicz, I., Mariconti, L., Tollervey, D., et al. (2010). RiboSys, a high-resolution, quantitative approach to measure the in vivo kinetics of pre-mRNA splicing and 3'-end processing in Saccharomyces cerevisiae. RNA 16, 2570-2580.

Alexandrov, A., Colognori, D., and Steitz, J.A. (2011). Human elF4AIII interacts with an elF4G-like partner, NOM1, revealing an evolutionarily conserved function outside the exon junction complex. Genes Dev. 25, 1078-1090.

Allmang, C., and Tollervey, D. (1998). The role of the 3' external transcribed spacer in yeast pre-rRNA processing. J. Mol. Biol. 278, 67-78.

Ameismeier, M., Cheng, J., Berninghausen, O., and Beckmann, R. (2018). Visualizing late states of human $40 S$ ribosomal subunit maturation. Nature 558, 249-253.

Andersen, C.B.F., Ballut, L., Johansen, J.S., Chamieh, H., Nielsen, K.H., Oliveira, C.L.P., Pedersen, J.S., Séraphin, B., Le Hir, H., and Andersen, G.R. (2006). Structure of the exon junction core complex with a trapped DEAD-box ATPase bound to RNA. Science 313, 1968-1972.

Andreou, A.Z., and Klostermeier, D. (2013). The DEAD-box helicase elF4A. RNA Biol. 10, 19-32.

Aravind, L., and Koonin, E. V. (1999). G-patch: a new conserved domain in eukaryotic RNA-processing proteins and type D retroviral polyproteins. Trends Biochem. Sci. 24, 342-344.

Ballut, L., Marchadier, B., Baguet, A., Tomasetto, C., Séraphin, B., and Le Hir, H. (2005). The exon junction core complex is locked onto RNA by inhibition of elF4AIII ATPase activity. Nat. Struct. Mol. Biol. 12, 861-869.

Ban, N., Nissen, P., Hansen, J., Moore, P.B., and Steitz, T.A. (2000). The Complete Atomic 
Structure of the Large Ribosomal Subunit at 2.4 A Resolution. Science (80-. ). 289, 905920.

Banerjee, D., McDaniel, P.M., and Rymond, B.C. (2015). Limited Portability of G-Patch Domains in Regulators of the Prp43 RNA Helicase Required for Pre-mRNA Splicing and Ribosomal RNA Maturation in Saccharomyces cerevisiae. Genetics 200, 135-147.

Banroques, J., Cordin, O., Doère, M., Linder, P., and Tanner, N.K. (2011). Analyses of the functional regions of DEAD-box RNA 'helicases' with deletion and chimera constructs tested in vivo and in vitro. J. Mol. Biol. 413, 451-472.

Barandun, J., Chaker-Margot, M., Hunziker, M., Molloy, K.R., Chait, B.T., and Klinge, S. (2017). The complete structure of the small-subunit processome. Nat. Struct. Mol. Biol. 24, 944-953.

Barandun, J., Hunziker, M., and Klinge, S. (2018). Assembly and structure of the SSU processome - a nucleolar precursor of the small ribosomal subunit. Curr. Opin. Struct. Biol. 49, 85-93.

Ben-Shem, A., Garreau de Loubresse, N., Melnikov, S., Jenner, L., Yusupova, G., and Yusupov, M. (2011). The Structure of the Eukaryotic Ribosome at 3.0 A Resolution. Science (80-. ). 334, 1524-1529.

Bohnsack, M.T., Kos, M., and Tollervey, D. (2008). Quantitative analysis of snoRNA association with pre-ribosomes and release of snR30 by Rok1 helicase. EMBO Rep. 9, $1230-1236$.

Bohnsack, M.T., Martin, R., Granneman, S., Ruprecht, M., Schleiff, E., and Tollervey, D. (2009). Prp43 Bound at Different Sites on the Pre-rRNA Performs Distinct Functions in Ribosome Synthesis. Mol. Cell 36, 583-592.

Bohnsack, M.T., Tollervey, D., and Granneman, S. (2012). Identification of RNA Helicase Target Sites by UV Cross-Linking and Analysis of cDNA. In Methods in Enzymology, pp. 275-288.

Bond, A.T., Mangus, D.A., He, F., and Jacobson, A. (2001). Absence of Dbp2p alters both nonsense-mediated mRNA decay and rRNA processing. Mol. Cell. Biol.

Booy, E.P., McRae, E.K.S., Howard, R., Deo, S.R., Ariyo, E.O., Dzananovic, E., Meier, M., Stetefeld, J., and McKenna, S.A. (2016). RNA Helicase Associated with AU-rich Element (RHAU/DHX36) Interacts with the 3'-Tail of the Long Non-coding RNA BC200 (BCYRN1). J. Biol. Chem. 291, 5355-5372.

Bourgeois, C.F., Mortreux, F., and Auboeuf, D. (2016). The multiple functions of RNA 
helicases as drivers and regulators of gene expression. Nat. Rev. Mol. Cell Biol. 17, 426438.

Bowers, H.A. (2006). Discriminatory RNP remodeling by the DEAD-box protein DED1. RNA 12, 903-912.

Brachmann, C.B., Davies, A., Cost, G.J., Caputo, E., Li, J., Hieter, P., and Boeke, J.D. (1998). Designer deletion strains derived fromSaccharomyces cerevisiae S288C: A useful set of strains and plasmids for PCR-mediated gene disruption and other applications. Yeast 14, 115-132.

Bradatsch, B., Leidig, C., Granneman, S., Gnädig, M., Tollervey, D., Böttcher, B., Beckmann, R., and Hurt, E. (2012). Structure of the pre-60S ribosomal subunit with nuclear export factor Arx1 bound at the exit tunnel. Nat. Struct. Mol. Biol. 19, 1234-1241.

Brüning, L., Hackert, P., Martin, R., Davila Gallesio, J., Aquino, G.R.R., Urlaub, H., Sloan, K.E., and Bohnsack, M.T. (2018). RNA helicases mediate structural transitions and compositional changes in pre-ribosomal complexes. Nat. Commun. 9, 5383.

Bruns, A.M., and Horvath, C.M. (2012). Activation of RIG-I-like receptor signal transduction. Crit. Rev. Biochem. Mol. Biol.

Buchwald, G., Schüssler, S., Basquin, C., Le Hir, H., Conti, E., Schuessler, S., Basquin, C., Hir, H. Le, and Conti, E. (2013). Crystal structure of the human elF4AIII-CWC22 complex shows how a DEAD-box protein is inhibited by a MIF4G domain. Proc. Natl. Acad. Sci. U. S. A. 110, E4611-E4618.

Caruthers, J.M., and McKay, D.B. (2002). Helicase structure and mechanism. Curr. Opin. Struct. Biol. 12, 123-133.

Cerezo, E., Plisson-Chastang, C., Henras, A.K., Lebaron, S., Gleizes, P., O'Donohue, M., Romeo, Y., and Henry, Y. (2019). Maturation of pre-40S particles in yeast and humans. Wiley Interdiscip. Rev. RNA 10, e1516.

Chaker-Margot, M., Hunziker, M., Barandun, J., Dill, B.D., and Klinge, S. (2015). Stagespecific assembly events of the 6-MDa small-subunit processome initiate eukaryotic ribosome biogenesis. Nat. Struct. Mol. Biol. 22, 920-923.

Chaker-Margot, M., Barandun, J., Hunziker, M., and Klinge, S. (2017). Architecture of the yeast small subunit processome. Science (80-. ). 355, eaal1880.

Chen, Y., Potratz, J.P., Tijerina, P., Del Campo, M., Lambowitz, A.M., and Russell, R. (2008). DEAD-box proteins can completely separate an RNA duplex using a single ATP. Proc. Natl. Acad. Sci. 105, 20203-20208. 
Choe, J., Ryu, I., Park, O.H., Park, J., Cho, H., Yoo, J.S., Chi, S.W., Kim, M.K., Song, H.K., and Kim, Y.K. (2014). elF4AIIl enhances translation of nuclear cap-binding complex-bound mRNAs by promoting disruption of secondary structures in 5'UTR. Proc. Natl. Acad. Sci. 111, E4577-E4586.

Choudhury, P., Hackert, P., Memet, I., Sloan, K.E., and Bohnsack, M.T. (2019). The human RNA helicase DHX37 is required for release of the U3 snoRNP from pre-ribosomal particles. RNA Biol. 16, 54-68.

Christian, H., Hofele, R. V., Urlaub, H., and Ficner, R. (2014). Insights into the activation of the helicase Prp43 by biochemical studies and structural mass spectrometry. Nucleic Acids Res. 42, 1162-1179.

Clark, E.L., Coulson, A., Dalgliesh, C., Rajan, P., Nicol, S.M., Fleming, S., Heer, R., Gaughan, L., Leung, H.Y., Elliott, D.J., et al. (2008). The RNA Helicase p68 Is a Novel Androgen Receptor Coactivator Involved in Splicing and Is Overexpressed in Prostate Cancer. Cancer Res. 68, 7938-7946.

Clark, E.L., Hadjimichael, C., Temperley, R., Barnard, A., Fuller-Pace, F. V., and Robson, C.N. (2013). p68/DdX5 Supports $\beta$-Catenin \&amp; RNAP II during Androgen Receptor Mediated Transcription in Prostate Cancer. PLoS One 8, e54150.

Cordin, O., Tanner, N.K., Doère, M., Linder, P., and Banroques, J. (2004). The newly discovered $Q$ motif of DEAD-box RNA helicases regulates RNA-binding and helicase activity. EMBO J. 23, 2478-2487.

Dembowski, J.A., Kuo, B., and Woolford, J.L. (2013). Has1 regulates consecutive maturation and processing steps for assembly of 605 ribosomal subunits. Nucleic Acids Res. 41, 7889-7904.

Diges, C.M. (2001). Escherichia coli DbpA is an RNA helicase that requires hairpin 92 of $23 S$ rRNA. EMBO J. 20, 5503-5512.

Dragon, F., Gallagher, J.E.G., Compagnone-Post, P.A., Mitchell, B.M., Porwancher, K.A., Wehner, K.A., Wormsley, S., Settlage, R.E., Shabanowitz, J., Osheim, Y., et al. (2002). A large nucleolar U3 ribonucleoprotein required for $18 \mathrm{~S}$ ribosomal RNA biogenesis. Nature 417, 967-970.

Edgcomb, S.P., Carmel, A.B., Naji, S., Ambrus-Aikelin, G., Reyes, J.R., Saphire, A.C.S., Gerace, L., and Williamson, J.R. (2012). DDX1 Is an RNA-Dependent ATPase Involved in HIV-1 Rev Function and Virus Replication. J. Mol. Biol. 415, 61-74.

Fairman-Williams, M.E., Guenther, U.-P., and Jankowsky, E. (2010). SF1 and SF2 
helicases: family matters. Curr. Opin. Struct. Biol. 20, 313-324.

Fairman, M.E. (2004). Protein Displacement by DExH/D 'RNA Helicases' Without Duplex Unwinding. Science (80-. ). 304, 730-734.

Falk, S., Tants, J.-N., Basquin, J., Thoms, M., Hurt, E., Sattler, M., and Conti, E. (2017). Structural insights into the interaction of the nuclear exosome helicase Mtr4 with the preribosomal protein Nop53. RNA 23, 1780-1787.

Fedorova, O., Solem, A., and Pyle, A.M. (2010). Protein-Facilitated Folding of Group II Intron Ribozymes. J. Mol. Biol. 397, 799-813.

Fernández-Pevida, A., Kressler, D., and de la Cruz, J. (2015). Processing of preribosomal RNA in Saccharomyces cerevisiae. Wiley Interdiscip. Rev. RNA 6, 191-209.

Fischer, N. (2002). The DEAD box protein Dhh1 stimulates the decapping enzyme Dcp1. EMBO J. 21, 2788-2797.

Fourmann, J.-B., Schmitzova, J., Christian, H., Urlaub, H., Ficner, R., Boon, K.-L., Fabrizio, P., and Luhrmann, R. (2013). Dissection of the factor requirements for spliceosome disassembly and the elucidation of its dissociation products using a purified splicing system. Genes Dev. 27, 413-428.

Fourmann, J.-B., Tauchert, M.J., Ficner, R., Fabrizio, P., and Lührmann, R. (2017). Regulation of Prp43-mediated disassembly of spliceosomes by its cofactors Ntr1 and Ntr2. Nucleic Acids Res. 45, 4068-4080.

Fuller-Pace, F. V. (2013). The DEAD box proteins DDX5 (p68) and DDX17 (p72): Multitasking transcriptional regulators. Biochim. Biophys. Acta - Gene Regul. Mech. 1829, 756763.

Gamalinda, M., Ohmayer, U., Jakovljevic, J., Kumcuoglu, B., Woolford, J., Mbom, B., Lin, L., and Woolford, J.L. (2014). A hierarchical model for assembly of eukaryotic 60S ribosomal subunit domains. Genes Dev. 28, 198-210.

Garcia-Garcia, C., Frieda, K.L., Feoktistova, K., Fraser, C.S., and Block, S.M. (2015). Factor-dependent processivity in human elF4A DEAD-box helicase. Science (80-. ). 348, $1486-1488$.

Geißler, V., Altmeyer, S., Stein, B., Uhlmann-Schiffler, H., and Stahl, H. (2013). The RNA helicase Ddx5/p68 binds to hUpf3 and enhances NMD of Ddx17/p72 and Smg5 mRNA. Nucleic Acids Res. 41, 7875-7888.

Gibson, D.G. (2011). Enzymatic Assembly of Overlapping DNA Fragments. In Methods in Enzymology, pp. 349-361. 
Gilman, B., Tijerina, P., and Russell, R. (2017). Distinct RNA-unwinding mechanisms of DEAD-box and DEAH-box RNA helicase proteins in remodeling structured RNAs and RNPs. Biochem. Soc. Trans. 45, 1313-1321.

Gómez-Herreros, F., Rodríguez-Galán, O., Morillo-Huesca, M., Maya, D., Arista-Romero, M., de la Cruz, J., Chávez, S., and Muñoz-Centeno, M.C. (2013). Balanced Production of Ribosome Components Is Required for Proper G 1 /S Transition in Saccharomyces cerevisiae. J. Biol. Chem. 288, 31689-31700.

Gómez-Herreros, F., Margaritis, T., Rodríguez-Galán, O., Pelechano, V., Begley, V., Millán-Zambrano, G., Morillo-Huesca, M., Muñoz-Centeno, M.C., Pérez-Ortín, J.E., de la Cruz, J., et al. (2017). The ribosome assembly gene network is controlled by the feedback regulation of transcription elongation. Nucleic Acids Res. 45, 9302-9318.

Granneman, S., Kudla, G., Petfalski, E., and Tollervey, D. (2009). Identification of protein binding sites on U3 snoRNA and pre-rRNA by UV cross-linking and high-throughput analysis of cDNAs. Proc. Natl. Acad. Sci. 106, 9613-9618.

Gross, T., Siepmann, A., Sturm, D., Windgassen, M., Scarcelli, J.J., Seedorf, M., Cole, C.N., and Krebber, H. (2007). The DEAD-Box RNA Helicase Dbp5 Functions in Translation Termination. Science (80-. ). 315, 646-649.

Gustafson, E.A., and Wessel, G.M. (2010). DEAD-box helicases: Posttranslational regulation and function. Biochem. Biophys. Res. Commun. 395, 1-6.

Halls, C., Mohr, S., Del Campo, M., Yang, Q., Jankowsky, E., and Lambowitz, A.M. (2007). Involvement of DEAD-box Proteins in Group I and Group II Intron Splicing. Biochemical Characterization of Mss116p, ATP Hydrolysis-dependent and -independent Mechanisms, and General RNA Chaperone Activity. J. Mol. Biol. 365, 835-855.

Hamann, F., Enders, M., and Ficner, R. (2019). Structural basis for RNA translocation by DEAH-box ATPases. Nucleic Acids Res. 47, 4349-4362.

Hardwick, S.W., and Luisi, B.F. (2013). Rarely at rest: RNA helicases and their busy contributions to RNA degradation, regulation and quality control. RNA Biol. 10, 56-70.

He, Y., Andersen, G.R., and Nielsen, K.H. (2010). Structural basis for the function of DEAH helicases. EMBO Rep. 11, 180-186.

He, Y., Staley, J.P., Andersen, G.R., and Nielsen, K.H. (2017). Structure of the DEAH/RHA ATPase Prp43p bound to RNA implicates a pair of hairpins and motif $\mathrm{Va}$ in translocation along RNA. RNA 23, 1110-1124.

Heerma van Voss, M.R., van Diest, P.J., and Raman, V. (2017). Targeting RNA helicases 
in cancer: The translation trap. Biochim. Biophys. Acta - Rev. Cancer 1868, 510-520.

Heininger, A.U., Hackert, P., Andreou, A.Z., Boon, K., Memet, I., Prior, M., Clancy, A., Schmidt, B., Urlaub, H., Schleiff, E., et al. (2016). Protein cofactor competition regulates the action of a multifunctional RNA helicase in different pathways. RNA Biol. 13, 320-330. Henras, A.K., Plisson-Chastang, C., O'Donohue, M.-F., Chakraborty, A., and Gleizes, P.E. (2015). An overview of pre-ribosomal RNA processing in eukaryotes. Wiley Interdiscip. Rev. RNA 6, 225-242.

Herschlag, D. (1995). RNA Chaperones and the RNA Folding Problem. J. Biol. Chem. 270, 20871-20874.

Hilbert, M., Karow, A.R., and Klostermeier, D. (2009). The mechanism of ATP-dependent RNA unwinding by DEAD box proteins. Biol. Chem. 390.

Hilbert, M., Kebbel, F., Gubaev, A., and Klostermeier, D. (2011). elF4G stimulates the activity of the DEAD box protein elF4A by a conformational guidance mechanism. Nucleic Acids Res. 39, 2260-2270.

Hooper, C., and Hilliker, A. (2013). Packing them up and dusting them off: RNA helicases and mRNA storage. Biochim. Biophys. Acta - Gene Regul. Mech. 1829, 824-834.

Hornbeck, P. V., Zhang, B., Murray, B., Kornhauser, J.M., Latham, V., and Skrzypek, E. (2015). PhosphoSitePlus, 2014: mutations, PTMs and recalibrations. Nucleic Acids Res. 43, D512-D520.

Hunziker, M., Barandun, J., Petfalski, E., Tan, D., Delan-Forino, C., Molloy, K.R., Kim, K.H., Dunn-Davies, H., Shi, Y., Chaker-Margot, M., et al. (2016). UtpA and UtpB chaperone nascent pre-ribosomal RNA and U3 snoRNA to initiate eukaryotic ribosome assembly. Nat. Commun. 7, 12090.

Jackson, R.J., Hellen, C.U.T., and Pestova, T. V. (2010). The mechanism of eukaryotic translation initiation and principles of its regulation. Nat. Rev. Mol. Cell Biol. 11, 113-127. Jacobs, A.-M., Nicol, S.M., Hislop, R.G., Jaffray, E.G., Hay, R.T., and Fuller-Pace, F. V. (2007). SUMO modification of the DEAD box protein p68 modulates its transcriptional activity and promotes its interaction with HDAC1. Oncogene 26, 5866-5876.

Janke, C., Magiera, M.M., Rathfelder, N., Taxis, C., Reber, S., Maekawa, H., MorenoBorchart, A., Doenges, G., Schwob, E., Schiebel, E., et al. (2004). A versatile toolbox for PCR-based tagging of yeast genes: new fluorescent proteins, more markers and promoter substitution cassettes. Yeast 21, 947-962.

Jankowsky, E. (2001). Active Disruption of an RNA-Protein Interaction by a DExH/D RNA 


\section{References}

Helicase. Science (80-. ). 291, 121-125.

Jarmoskaite, I., and Russell, R. (2014). RNA Helicase Proteins as Chaperones and Remodelers. Annu. Rev. Biochem. 83, 697-725.

Johnson, S.J., and Jackson, R.N. (2013). Ski2-like RNA helicase structures. RNA Biol. 10, 33-43.

Kappel, L., Loibl, M., Zisser, G., Klein, I., Fruhmann, G., Gruber, C., Unterweger, S., Rechberger, G., Pertschy, B., and Bergler, H. (2012). Rlp24 activates the AAA-ATPase Drg1 to initiate cytoplasmic pre-60S maturation. J. Cell Biol. 199, 771-782.

Karbstein, K. (2013). Quality control mechanisms during ribosome maturation. Trends Cell Biol. 23, 242-250.

Karunatilaka, K.S., Solem, A., Pyle, A.M., and Rueda, D. (2010). Single-molecule analysis of Mss116-mediated group II intron folding. Nature 467, 935-939.

Kater, L., Thoms, M., Barrio-Garcia, C., Cheng, J., Ismail, S., Ahmed, Y.L., Bange, G., Kressler, D., Berninghausen, O., Sinning, I., et al. (2017). Visualizing the Assembly Pathway of Nucleolar Pre-60S Ribosomes. Cell 171, 1599-1610.e14.

Khoshnevis, S., Askenasy, I., Johnson, M.C., Dattolo, M.D., Young-Erdos, C.L., Stroupe, M.E., and Karbstein, K. (2016). The DEAD-box Protein Rok1 Orchestrates 40S and 60S Ribosome Assembly by Promoting the Release of Rrp5 from Pre-40S Ribosomes to Allow for 60S Maturation. PLOS Biol. 14, e1002480.

Kiianitsa, K., Solinger, J.A., and Heyer, W.-D. (2003). NADH-coupled microplate photometric assay for kinetic studies of ATP-hydrolyzing enzymes with low and high specific activities. Anal. Biochem. 321, 266-271.

Kistler, A.L. (2001). Deletion of MUD2, the yeast homolog of U2AF65, can bypass the requirement for Sub2, an essential spliceosomal ATPase. Genes Dev. 15, 42-49.

Klinge, S., and Woolford, J.L. (2019). Ribosome assembly coming into focus. Nat. Rev. Mol. Cell Biol. 20, 116-131.

Klinge, S., Voigts-Hoffmann, F., Leibundgut, M., Arpagaus, S., and Ban, N. (2011). Crystal Structure of the Eukaryotic 60S Ribosomal Subunit in Complex with Initiation Factor 6. Science (80-. ). 334, 941-948.

Kornprobst, M., Turk, M., Kellner, N., Cheng, J., Flemming, D., Koš-Braun, I., Koš, M., Thoms, M., Berninghausen, O., Beckmann, R., et al. (2016). Architecture of the 90S Preribosome: A Structural View on the Birth of the Eukaryotic Ribosome. Cell 166, 380-393.

Kos-Braun, I.C., Jung, I., and Koš, M. (2017). Tor1 and CK2 kinases control a switch 
between alternative ribosome biogenesis pathways in a growth-dependent manner. PLOS Biol. 15, e2000245.

Koš, M., and Tollervey, D. (2005). The Putative RNA Helicase Dbp4p Is Required for Release of the U14 snoRNA from Preribosomes in Saccharomyces cerevisiae. Mol. Cell 20, 53-64.

Koš, M., and Tollervey, D. (2010). Yeast Pre-rRNA Processing and Modification Occur Cotranscriptionally. Mol. Cell 37, 809-820.

Kressler, D., de la Cruz, J., Rojo, M., and Linder, P. (1997). Fal1p is an essential DEADbox protein involved in 40S-ribosomal-subunit biogenesis in Saccharomyces cerevisiae. Mol. Cell. Biol. 17, 7283-7294.

Kressler, D., Linder, P., and de la Cruz, J. (1999). Protein trans -Acting Factors Involved in Ribosome Biogenesis in Saccharomyces cerevisiae. Mol. Cell. Biol. 19, 7897-7912.

Kressler, D., Hurt, E., Bergler, H., and Baßler, J. (2012). The power of AAA-ATPases on the road of pre-60S ribosome maturation - Molecular machines that strip pre-ribosomal particles. Biochim. Biophys. Acta - Mol. Cell Res. 1823, 92-100.

Kretschmer, J., Rao, H., Hackert, P., Sloan, K.E., Höbartner, C., and Bohnsack, M.T. (2018). The m6A reader protein YTHDC2 interacts with the small ribosomal subunit and the 5'-3' exoribonuclease XRN1. RNA 24, 1339-1350.

Krishnan, V., and Zeichner, S.L. (2004). Alterations in the expression of DEAD-box and other RNA binding proteins during HIV-1 replication. Retrovirology 1, 42.

Kudla, G., Granneman, S., Hahn, D., Beggs, J.D., and Tollervey, D. (2011). Cross-linking, ligation, and sequencing of hybrids reveals RNA-RNA interactions in yeast. Proc. Natl. Acad. Sci. 108, 10010-10015.

de la Cruz, J., Gómez-Herreros, F., Rodríguez-Galán, O., Begley, V., de la Cruz MuñozCenteno, M., and Chávez, S. (2018). Feedback regulation of ribosome assembly. Curr. Genet. 64, 393-404.

Lebaron, S., Schneider, C., van Nues, R.W., Swiatkowska, A., Walsh, D., Böttcher, B., Granneman, S., Watkins, N.J., and Tollervey, D. (2012). Proofreading of pre-40S ribosome maturation by a translation initiation factor and $60 S$ subunits. Nat. Struct. Mol. Biol. 19, 744-753.

Liang, X. -h., and Fournier, M.J. (2006). The Helicase Has1p Is Required for snoRNA Release from Pre-rRNA. Mol. Cell. Biol. 26, 7437-7450.

Lin, D., Pestova, T. V., Hellen, C.U.T., and Tiedge, H. (2008). Translational Control by a 
Small RNA: Dendritic BC1 RNA Targets the Eukaryotic Initiation Factor 4A Helicase Mechanism. Mol. Cell. Biol. 28, 3008-3019.

Linder, P., and Jankowsky, E. (2011). From unwinding to clamping - the DEAD box RNA helicase family. Nat. Rev. Mol. Cell Biol. 12, 505-516.

Linder, P., Lasko, P.F., Ashburner, M., Leroy, P., Nielsen, P.J., Nishi, K., Schnier, J., and Slonimski, P.P. (1989). Birth of the D-E-A-D box. Nature 337, 121-122.

Liu, F., Putnam, A., and Jankowsky, E. (2008). ATP hydrolysis is required for DEAD-box protein recycling but not for duplex unwinding. Proc. Natl. Acad. Sci. 105, 20209-20214.

Liu, F., Putnam, A.A., and Jankowsky, E. (2014a). DEAD-Box Helicases Form NucleotideDependent, Long-Lived Complexes with RNA. Biochemistry 53, 423-433.

Liu, Y., Lu, N., Yuan, B., Weng, L., Wang, F., Liu, Y.-J., and Zhang, Z. (2014b). The interaction between the helicase DHX33 and IPS-1 as a novel pathway to sense doublestranded RNA and RNA viruses in myeloid dendritic cells. Cell. Mol. Immunol. 11, 49-57. Liu, Z., Wang, Y., Gao, T., Pan, Z., Cheng, H., Yang, Q., Cheng, Z., Guo, A., Ren, J., and Xue, Y. (2014c). CPLM: a database of protein lysine modifications. Nucleic Acids Res. 42, D531-D536.

Lo, K.-Y., Li, Z., Bussiere, C., Bresson, S., Marcotte, E.M., and Johnson, A.W. (2010). Defining the Pathway of Cytoplasmic Maturation of the 60S Ribosomal Subunit. Mol. Cell 39, 196-208.

Lund, M.K., and Guthrie, C. (2005). The DEAD-Box Protein Dbp5p Is Required to Dissociate Mex67p from Exported mRNPs at the Nuclear Rim. Mol. Cell 20, 645-651.

Ma, C., Wu, S., Li, N., Chen, Y., Yan, K., Li, Z., Zheng, L., Lei, J., Woolford, J.L., and Gao, N. (2017). Structural snapshot of cytoplasmic pre-60S ribosomal particles bound by Nmd3, Lsg1, Tif6 and Reh1. Nat. Struct. Mol. Biol. 24, 214-220.

Ma, W.K., Paudel, B.P., Xing, Z., Sabath, I.G., Rueda, D., and Tran, E.J. (2016). Recruitment, Duplex Unwinding and Protein-Mediated Inhibition of the Dead-Box RNA Helicase Dbp2 at Actively Transcribed Chromatin. J. Mol. Biol. 428, 1091-1106.

Mallam, A.L., Jarmoskaite, I., Tijerina, P., Del Campo, M., Seifert, S., Guo, L., Russell, R., and Lambowitz, A.M. (2011). Solution structures of DEAD-box RNA chaperones reveal conformational changes and nucleic acid tethering by a basic tail. Proc. Natl. Acad. Sci. $108,12254-12259$.

Mallam, A.L., Del Campo, M., Gilman, B., Sidote, D.J., and Lambowitz, A.M. (2012). Structural basis for RNA-duplex recognition and unwinding by the DEAD-box helicase 
Mss116p. Nature 490, 121-125.

Manikas, R.-G., Thomson, E., Thoms, M., and Hurt, E. (2016). The K + -dependent GTPase Nug1 is implicated in the association of the helicase Dbp10 to the immature peptidyl transferase centre during ribosome maturation. Nucleic Acids Res. 44, 18001812.

Marintchev, A., and Wagner, G. (2005). elF4G and CBP80 Share a Common Origin and Similar Domain Organization: Implications for the Structure and Function of elF4G $\dagger$. Biochemistry 44, 12265-12272.

Martin, R., Straub, A.U., Doebele, C., and Bohnsack, M.T. (2013). DExD/H-box RNA helicases in ribosome biogenesis. RNA Biol. 10, 4-18.

Martin, R., Hackert, P., Ruprecht, M., Simm, S., Brüning, L., Mirus, O., Sloan, K.E., Kudla, G., Schleiff, E., and Bohnsack, M.T. (2014). A pre-ribosomal RNA interaction network involving snoRNAs and the Rok1 helicase. RNA 20, 1173-1182.

Mathys, H., Basquin, J., Ozgur, S., Czarnocki-Cieciura, M., Bonneau, F., Aartse, A., Dziembowski, A., Nowotny, M., Conti, E., and Filipowicz, W. (2014). Structural and Biochemical Insights to the Role of the CCR4-NOT Complex and DDX6 ATPase in MicroRNA Repression. Mol. Cell 54, 751-765.

Matsuo, Y., Granneman, S., Thoms, M., Manikas, R.-G., Tollervey, D., and Hurt, E. (2014). Coupled GTPase and remodelling ATPase activities form a checkpoint for ribosome export. Nature 505, 112-116.

Meier-Stephenson, V., Mrozowich, T., Pham, M., and Patel, T.R. (2018). DEAD-box helicases: the Yin and Yang roles in viral infections. Biotechnol. Genet. Eng. Rev. 34, 332.

Melnikov, S., Ben-Shem, A., Garreau de Loubresse, N., Jenner, L., Yusupova, G., and Yusupov, M. (2012). One core, two shells: bacterial and eukaryotic ribosomes. Nat. Struct. Mol. Biol. 19, 560-567.

Memet, I., Doebele, C., Sloan, K.E., and Bohnsack, M.T. (2017). The G-patch protein NF$\mathrm{KB}$-repressing factor mediates the recruitment of the exonuclease XRN2 and activation of the RNA helicase DHX15 in human ribosome biogenesis. Nucleic Acids Res. 45, 53595374 .

Miller, O.L., and Beatty, B.R. (1969). Visualization of Nucleolar Genes. Science (80-. ). 164, 955-957.

von Moeller, H., Basquin, C., and Conti, E. (2009). The mRNA export protein DBP5 binds 
RNA and the cytoplasmic nucleoporin NUP214 in a mutually exclusive manner. Nat. Struct. Mol. Biol. 16, 247-254.

Montpetit, B., Thomsen, N.D., Helmke, K.J., Seeliger, M.A., Berger, J.M., and Weis, K. (2011). A conserved mechanism of DEAD-box ATPase activation by nucleoporins and InsP6 in mRNA export. Nature 472, 238-242.

Morawska, M., and Ulrich, H.D. (2013). An expanded tool kit for the auxin-inducible degron system in budding yeast. Yeast 30, 341-351.

Mori, M., Triboulet, R., Mohseni, M., Schlegelmilch, K., Shrestha, K., Camargo, F.D., and Gregory, R.I. (2014). Hippo Signaling Regulates Microprocessor and Links Cell-DensityDependent miRNA Biogenesis to Cancer. Cell 156, 893-906.

Moriggi, G., Nieto, B., and Dosil, M. (2014). Rrp12 and the Exportin Crm1 Participate in Late Assembly Events in the Nucleolus during 40S Ribosomal Subunit Biogenesis. PLoS Genet. 10, e1004836.

Morino, S., Imataka, H., Svitkin, Y. V, Pestova, T. V, and Sonenberg, N. (2000). Eukaryotic translation initiation factor 4E (elF4E) binding site and the middle one-third of elF4GI constitute the core domain for cap-dependent translation, and the C-terminal one-third functions as a modulatory region. Mol. Cell. Biol. 20, 468-477.

Motiño, O., Francés, D.E., Mayoral, R., Castro-Sánchez, L., Fernández-Velasco, M., Boscá, L., García-Monzón, C., Brea, R., Casado, M., Agra, N., et al. (2015). Regulation of MicroRNA 183 by Cyclooxygenase 2 in Liver Is DEAD-Box Helicase p68 (DDX5) Dependent: Role in Insulin Signaling. Mol. Cell. Biol. 35, 2554-2567.

Mougey, E.B., O’Reilly, M., Osheim, Y., Miller, O.L., Beyer, A., and Sollner-Webb, B. (1993). The terminal balls characteristic of eukaryotic rRNA transcription units in chromatin spreads are rRNA processing complexes. Genes Dev. 7, 1609-1619.

Mugler, C.F., Hondele, M., Heinrich, S., Sachdev, R., Vallotton, P., Koek, A.Y., Chan, L.Y., and Weis, K. (2016). ATPase activity of the DEAD-box protein Dhh1 controls processing body formation. Elife 5 .

Nerurkar, P., Altvater, M., Gerhardy, S., Schütz, S., Fischer, U., Weirich, C., and Panse, V.G. (2015). Eukaryotic Ribosome Assembly and Nuclear Export. In International Review of Cell and Molecular Biology, pp. 107-140.

Nielsen, K.H., Chamieh, H., Andersen, C.B.F., Fredslund, F., Hamborg, K., Le Hir, H., and Andersen, G.R. (2008). Mechanism of ATP turnover inhibition in the EJC. RNA 15, 67-75. Noble, C.G., and Song, H. (2007). MLN51 Stimulates the RNA-Helicase Activity of 
elF4AIII. PLoS One 2, e303.

Osheim, Y.N., French, S.L., Keck, K.M., Champion, E.A., Spasov, K., Dragon, F., Baserga, S.J., and Beyer, A.L. (2004). Pre-18S Ribosomal RNA Is Structurally Compacted into the SSU Processome Prior to Being Cleaved from Nascent Transcripts in Saccharomyces cerevisiae. Mol. Cell 16, 943-954.

Ozgur, S., Buchwald, G., Falk, S., Chakrabarti, S., Prabu, J.R., and Conti, E. (2015). The conformational plasticity of eukaryotic RNA-dependent ATPases. FEBS J. 282, 850-863.

Pause, A., and Sonenberg, N. (1992). Mutational analysis of a DEAD box RNA helicase: the mammalian translation initiation factor elF-4A. EMBO J. 11, 2643-2654.

Peña, C., Hurt, E., and Panse, V.G. (2017). Eukaryotic ribosome assembly, transport and quality control. Nat. Struct. Mol. Biol. 24, 689-699.

Pérez-Fernández, J., Roman, A., De Las Rivas, J., Bustelo, X.R., and Dosil, M. (2007). The $90 \mathrm{~S}$ Preribosome is a Multimodular Structure That Is Assembled through a Hierarchical Mechanism. Mol. Cell. Biol. 27, 5414-5429.

Pérez-Fernández, J., Martín-Marcos, P., and Dosil, M. (2011). Elucidation of the assembly events required for the recruitment of Utp20, Imp4 and Bms1 onto nascent pre-ribosomes. Nucleic Acids Res. 39, 8105-8121.

Pertschy, B., Schneider, C., Gnädig, M., Schäfer, T., Tollervey, D., and Hurt, E. (2009). RNA Helicase Prp43 and Its Co-factor Pfa1 Promote 20 to 18 S rRNA Processing Catalyzed by the Endonuclease Nob1. J. Biol. Chem. 284, 35079-35091.

Petrov, A.S., Bernier, C.R., Gulen, B., Waterbury, C.C., Hershkovits, E., Hsiao, C., Harvey, S.C., Hud, N. V., Fox, G.E., Wartell, R.M., et al. (2014). Secondary Structures of rRNAs from All Three Domains of Life. PLoS One 9, e88222.

Pillet, B., Mitterer, V., Kressler, D., and Pertschy, B. (2017). Hold on to your friends: Dedicated chaperones of ribosomal proteins. BioEssays 39, e201600153.

Ponting, C.P. (2000). Novel elF4G domain homologues linking mRNA translation with nonsense-mediated mRNA decay. Trends Biochem. Sci. 25, 423-426.

Rabl, J., Leibundgut, M., Ataide, S.F., Haag, A., and Ban, N. (2011). Crystal Structure of the Eukaryotic 40S Ribosomal Subunit in Complex with Initiation Factor 1. Science (80-. ). $331,730-736$.

Rawling, D.C., and Pyle, A.M. (2014). Parts, assembly and operation of the RIG-I family of motors. Curr. Opin. Struct. Biol. 25, 25-33.

Robert-Paganin, J., Réty, S., and Leulliot, N. (2015). Regulation of DEAH/RHA Helicases 
by G-Patch Proteins. Biomed Res. Int. 2015, 1-9.

Robert-Paganin, J., Halladjian, M., Blaud, M., Lebaron, S., Delbos, L., Chardon, F., Capeyrou, R., Humbert, O., Henry, Y., Henras, A.K., et al. (2017). Functional link between DEAH/RHA helicase Prp43 activation and ATP base binding. Nucleic Acids Res. 45, 1539 1552.

Rosado, I. V, Dez, C., Lebaron, S., Caizergues-Ferrer, M., Henry, Y., and de la Cruz, J. (2007). Characterization of Saccharomyces cerevisiae Npa2p (Urb2p) Reveals a LowMolecular-Mass Complex Containing Dbp6p, Npa1p (Urb1p), Nop8p, and Rsa3p Involved in Early Steps of 60S Ribosomal Subunit Biogenesis. Mol. Cell. Biol. 27, 1207-1221.

Rudolph, M.G., and Klostermeier, D. (2015). When core competence is not enough: functional interplay of the DEAD-box helicase core with ancillary domains and auxiliary factors in RNA binding and unwinding. Biol. Chem. 396.

Samatanga, B., and Klostermeier, D. (2014). DEAD-box RNA helicase domains exhibit a continuum between complete functional independence and high thermodynamic coupling in nucleotide and RNA duplex recognition. Nucleic Acids Res. 42, 10644-10654.

Sambrook, J., and W Russell, D. (2001). Molecular Cloning: A Laboratory Manual. Cold Spring Harb. Lab. Press. Cold Spring Harb. NY 999.

Sardana, R., Liu, X., Granneman, S., Zhu, J., Gill, M., Papoulas, O., Marcotte, E.M., Tollervey, D., Correll, C.C., and Johnson, A.W. (2015). The DEAH-box Helicase Dhr1 Dissociates U3 from the Pre-rRNA to Promote Formation of the Central Pseudoknot. PLOS Biol. 13, e1002083.

Schuller, J.M., Falk, S., Fromm, L., Hurt, E., and Conti, E. (2018). Structure of the nuclear exosome captured on a maturing preribosome. Science (80-. ). 360, 219-222.

Schutz, P., Bumann, M., Oberholzer, A.E., Bieniossek, C., Trachsel, H., Altmann, M., and Baumann, U. (2008). Crystal structure of the yeast elF4A-elF4G complex: An RNAhelicase controlled by protein-protein interactions. Proc. Natl. Acad. Sci. 105, 9564-9569.

Schwer, B. (2008). A Conformational Rearrangement in the Spliceosome Sets the Stage for Prp22-Dependent mRNA Release. Mol. Cell 30, 743-754.

Semlow, D.R., and Staley, J.P. (2012). Staying on message: Ensuring fidelity in pre-mRNA splicing. Trends Biochem. Sci.

Semlow, D.R., Blanco, M.R., Walter, N.G., and Staley, J.P. (2016). Spliceosomal DEAHBox ATPases Remodel Pre-mRNA to Activate Alternative Splice Sites. Cell 164, 985-998. Sharif, H., Ozgur, S., Sharma, K., Basquin, C., Urlaub, H., and Conti, E. (2013). Structural 
analysis of the yeast Dhh1-Pat1 complex reveals how Dhh1 engages Pat1, Edc3 and RNA in mutually exclusive interactions. Nucleic Acids Res. 41, 8377-8390.

Sharma, S., and Lafontaine, D.L.J. (2015). 'View From A Bridge': A New Perspective on Eukaryotic rRNA Base Modification. Trends Biochem. Sci. 40, 560-575.

Sharma, S., Langhendries, J.-L., Watzinger, P., Kötter, P., Entian, K.-D., and Lafontaine, D.L.J. (2015). Yeast Kre33 and human NAT10 are conserved 18S rRNA cytosine acetyltransferases that modify tRNAs assisted by the adaptor Tan1/THUMPD1. Nucleic Acids Res. 43, 2242-2258.

Sharma, S., Yang, J., van Nues, R., Watzinger, P., Kötter, P., Lafontaine, D.L.J., Granneman, S., and Entian, K.-D. (2017). Specialized box C/D snoRNPs act as antisense guides to target RNA base acetylation. PLOS Genet. 13, e1006804.

Shen, H., Zheng, X., Shen, J., Zhang, L., Zhao, R., and Green, M.R. (2008). Distinct activities of the DExD/H-box splicing factor hUAP56 facilitate stepwise assembly of the spliceosome. Genes Dev. 22, 1796-1803.

Singleton, M.R., Dillingham, M.S., and Wigley, D.B. (2007). Structure and Mechanism of Helicases and Nucleic Acid Translocases. Annu. Rev. Biochem. 76, 23-50.

Sloan, K.E., and Bohnsack, M.T. (2018). Unravelling the Mechanisms of RNA Helicase Regulation. Trends Biochem. Sci. 43, 237-250.

Sloan, K.E., Warda, A.S., Sharma, S., Entian, K.-D., Lafontaine, D.L.J., and Bohnsack, M.T. (2017). Tuning the ribosome: The influence of rRNA modification on eukaryotic ribosome biogenesis and function. RNA Biol. 14, 1138-1152.

Soltanieh, S., Osheim, Y.N., Spasov, K., Trahan, C., Beyer, A.L., and Dragon, F. (2015). DEAD-Box RNA Helicase Dbp4 Is Required for Small-Subunit Processome Formation and Function. Mol. Cell. Biol. 35, 816-830.

Song, C., Hotz-Wagenblatt, A., Voit, R., and Grummt, I. (2017). SIRT7 and the DEAD-box helicase DDX21 cooperate to resolve genomic $R$ loops and safeguard genome stability. Genes Dev. 31, 1370-1381.

Soulat, D., Bürckstümmer, T., Westermayer, S., Goncalves, A., Bauch, A., Stefanovic, A., Hantschel, O., Bennett, K.L., Decker, T., and Superti-Furga, G. (2008). The DEAD-box helicase DDX3X is a critical component of the TANK-binding kinase 1-dependent innate immune response. EMBO J. 27, 2135-2146.

Steckelberg, A.-L., Boehm, V., Gromadzka, A.M., and Gehring, N.H. (2012). CWC22 Connects Pre-mRNA Splicing and Exon Junction Complex Assembly. Cell Rep. 2, 454- 
461.

Steckelberg, A.-L., Altmueller, J., Dieterich, C., and Gehring, N.H. (2015). CWC22dependent pre-mRNA splicing and elF4A3 binding enables global deposition of exon junction complexes. Nucleic Acids Res. 43, 4687-4700.

Steimer, L., and Klostermeier, D. (2012). RNA helicases in infection and disease. RNA Biol. 9, 751-771.

Strunk, B.S., Novak, M.N., Young, C.L., and Karbstein, K. (2012). A Translation-Like Cycle Is a Quality Control Checkpoint for Maturing 40S Ribosome Subunits. Cell 150, 111-121.

Sun, Q., Zhu, X., Qi, J., An, W., Lan, P., Tan, D., Chen, R., Wang, B., Zheng, S., Zhang, C., et al. (2017). Molecular architecture of the $90 \mathrm{~S}$ small subunit pre-ribosome. Elife 6.

Suzuki, H.I., Yamagata, K., Sugimoto, K., Iwamoto, T., Kato, S., and Miyazono, K. (2009). Modulation of microRNA processing by p53. Nature 460, 529-533.

Svitkin, Y. V, Pause, A., Haghighat, A., Pyronnet, S., Witherell, G., Belsham, G.J., and Sonenberg, N. (2001). The requirement for eukaryotic initiation factor 4A (elF4A) in translation is in direct proportion to the degree of mRNA 5' secondary structure. RNA 7, 382-394.

Swiatkowska, A., Wlotzka, W., Tuck, A., Barrass, J.D., Beggs, J.D., and Tollervey, D. (2012). Kinetic analysis of pre-ribosome structure in vivo. RNA 18, 2187-2200.

Talkish, J., Biedka, S., Jakovljevic, J., Zhang, J., Tang, L., Strahler, J.R., Andrews, P.C., Maddock, J.R., and Woolford, J.L. (2016). Disruption of ribosome assembly in yeast blocks cotranscriptional pre-rRNA processing and affects the global hierarchy of ribosome biogenesis. RNA 22, 852-866.

Tanner, N.K., Cordin, O., Banroques, J., Doère, M., and Linder, P. (2003). The Q Motif. Mol. Cell 11, 127-138.

Tauchert, M.J., Fourmann, J.-B., Lührmann, R., and Ficner, R. (2017). Structural insights into the mechanism of the DEAH-box RNA helicase Prp43. Elife 6.

Tedeschi, F.A., Cloutier, S.C., Tran, E.J., and Jankowsky, E. (2018). The DEAD-box protein Dbp2p is linked to noncoding RNAs, the helicase Sen1p, and R-loops. RNA 24, 1693-1705.

Thoms, M., Thomson, E., Baßler, J., Gnädig, M., Griesel, S., and Hurt, E. (2015). The Exosome Is Recruited to RNA Substrates through Specific Adaptor Proteins. Cell 162, 1029-1038.

Thomson, E., and Tollervey, D. (2010). The Final Step in 5.8S rRNA Processing Is 
Cytoplasmic in Saccharomyces cerevisiae. Mol. Cell. Biol. 30, 976-984.

Tieg, B., and Krebber, H. (2013). Dbp5 - From nuclear export to translation. Biochim. Biophys. Acta - Gene Regul. Mech. 1829, 791-798.

Torreira, E., Louro, J.A., Pazos, I., González-Polo, N., Gil-Carton, D., Duran, A.G., Tosi, S., Gallego, O., Calvo, O., and Fernández-Tornero, C. (2017). The dynamic assembly of distinct RNA polymerase I complexes modulates rDNA transcription. Elife 6 .

Tran, E.J., Zhou, Y., Corbett, A.H., and Wente, S.R. (2007). The DEAD-Box Protein Dbp5 Controls mRNA Export by Triggering Specific RNA:Protein Remodeling Events. Mol. Cell $28,850-859$.

Turner, A.J., Knox, A.A., and Watkins, N.J. (2012). Nucleolar disruption leads to the spatial separation of key $18 \mathrm{~S}$ rRNA processing factors. RNA Biol. 9, 175-186.

Turowski, T.W., and Tollervey, D. (2015). Cotranscriptional events in eukaryotic ribosome synthesis. Wiley Interdiscip. Rev. RNA 6, 129-139.

Uhlmann-Schiffler, H. (2006). Ddx42p--a human DEAD box protein with RNA chaperone activities. Nucleic Acids Res. 34, 10-22.

Vanrobays, E., Leplus, A., Osheim, Y.N., Beyer, A.L., Wacheul, L., and Lafontaine, D.L.J. (2008). TOR regulates the subcellular distribution of DIM2, a KH domain protein required for cotranscriptional ribosome assembly and pre-40S ribosome export. RNA 14, 20612073.

Venema, J., Vos, H.R., Faber, A.W., van Venrooij, W.J., and Raué, H.A. (2000). Yeast Rrp9p is an evolutionarily conserved U3 snoRNP protein essential for early pre-rRNA processing cleavages and requires box $C$ for its association. RNA 6, 1660-1671.

Vincent, N.G., Charette, J.M., and Baserga, S.J. (2018). The SSU processome interactome in Saccharomyces cerevisiae reveals novel protein subcomplexes. RNA 24, 77-89.

Walbott, H., Mouffok, S., Capeyrou, R., Lebaron, S., Humbert, O., van Tilbeurgh, H., Henry, Y., and Leulliot, N. (2010). Prp43p contains a processive helicase structural architecture with a specific regulatory domain. EMBO J. 29, 2194-2204.

Waliullah, T.M., Yeasmin, A.M.S.T., Kaneko, A., Koike, N., Terasawa, M., Totsuka, T., and Ushimaru, T. (2017). Rim15 and Sch9 kinases are involved in induction of autophagic degradation of ribosomes in budding yeast. Biosci. Biotechnol. Biochem. 81, 307-310.

Walker, J.E., Saraste, M., Runswick, M.J., and Gay, N.J. (1982). Distantly related sequences in the alpha- and beta-subunits of ATP synthase, myosin, kinases and other 
ATP-requiring enzymes and a common nucleotide binding fold. EMBO J. 1, 945-951.

Warkocki, Z., Schneider, C., Mozaffari-Jovin, S., Schmitzová, J., Höbartner, C., Fabrizio, P., and Lührmann, R. (2015). The G-patch protein Spp2 couples the spliceosomestimulated ATPase activity of the DEAH-box protein Prp2 to catalytic activation of the spliceosome. Genes Dev. 29, 94-107.

Warner, J.R. (1999). The economics of ribosome biosynthesis in yeast. Trends Biochem. Sci. 24, 437-440.

Watkins, N.J., and Bohnsack, M.T. (2012). The box C/D and H/ACA snoRNPs: key players in the modification, processing and the dynamic folding of ribosomal RNA. Wiley Interdiscip. Rev. RNA 3, 397-414.

Weaver, P.L., Sun, C., and Chang, T.H. (1997). Dbp3p, a putative RNA helicase in Saccharomyces cerevisiae, is required for efficient pre-rRNA processing predominantly at site A3. Mol. Cell. Biol. 17, 1354-1365.

Webb, S., Hector, R.D., Kudla, G., and Granneman, S. (2014). PAR-CLIP data indicate that Nrd1-Nab3-dependent transcription termination regulates expression of hundreds of protein coding genes in yeast. Genome Biol. 15, R8.

Wells, G.R., Weichmann, F., Colvin, D., Sloan, K.E., Kudla, G., Tollervey, D., Watkins, N.J., and Schneider, C. (2016). The PIN domain endonuclease Utp24 cleaves preribosomal RNA at two coupled sites in yeast and humans. Nucleic Acids Res.

Wimberly, B.T., Brodersen, D.E., Clemons, W.M., Morgan-Warren, R.J., Carter, A.P., Vonrhein, C., Hartsch, T., and Ramakrishnan, V. (2000). Structure of the 30 S ribosomal subunit. Nature 407, 327-339.

Wojtas, M.N., Pandey, R.R., Mendel, M., Homolka, D., Sachidanandam, R., and Pillai, R.S. (2017). Regulation of m6A Transcripts by the $3^{\prime} \rightarrow 5^{\prime}$ RNA Helicase YTHDC2 Is Essential for a Successful Meiotic Program in the Mammalian Germline. Mol. Cell 68, 374-387.e12. Wu, S., Tutuncuoglu, B., Yan, K., Brown, H., Zhang, Y., Tan, D., Gamalinda, M., Yuan, Y., Li, Z., Jakovljevic, J., et al. (2016). Diverse roles of assembly factors revealed by structures of late nuclear pre-60S ribosomes. Nature 534, 133-137.

Xing, Z., Ma, W.K., and Tran, E.J. (2019). The DDX5/Dbp2 subfamily of DEAD-box RNA helicases. Wiley Interdiscip. Rev. RNA 10, e1519.

Yang, Q., and Jankowsky, E. (2005). ATP- and ADP-Dependent Modulation of RNA Unwinding and Strand Annealing Activities by the DEAD-Box Protein DED1 †. Biochemistry 44, 13591-13601. 
Yang, Q., Del Campo, M., Lambowitz, A.M., and Jankowsky, E. (2007a). DEAD-Box Proteins Unwind Duplexes by Local Strand Separation. Mol. Cell 28, 253-263.

Yang, Q., Fairman, M.E., and Jankowsky, E. (2007b). DEAD-box-protein-assisted RNA Structure Conversion Towards and Against Thermodynamic Equilibrium Values. J. Mol. Biol. 368, 1087-1100.

Yeh, T.-C., Liu, H.-L., Chung, C.-S., Wu, N.-Y., Liu, Y.-C., and Cheng, S.-C. (2011). Splicing Factor Cwc22 Is Required for the Function of Prp2 and for the Spliceosome To Escape from a Futile Pathway. Mol. Cell. Biol. 31, 43-53.

Yusupov, M.M., Yusupova, G.Z., Baucom, A., Lieberman, K., Earnest, T.N., Cate, J.H., and Noller, H.F. (2001). Crystal structure of the ribosome at $5.5 \mathrm{~A}$ resolution. Science 292, 883-896.

Zhang, Z., Yuan, B., Lu, N., Facchinetti, V., and Liu, Y.-J. (2011). DHX9 Pairs with IPS-1 To Sense Double-Stranded RNA in Myeloid Dendritic Cells. J. Immunol. 187, 4501-4508.

Zhou, D., Zhu, X., Zheng, S., Tan, D., Dong, M.-Q., and Ye, K. (2019). Cryo-EM structure of an early precursor of large ribosomal subunit reveals a half-assembled intermediate. Protein Cell 10, 120-130.

Zhu, J., Liu, X., Anjos, M., Correll, C.C., and Johnson, A.W. (2016). Utp14 Recruits and Activates the RNA Helicase Dhr1 To Undock U3 snoRNA from the Preribosome. Mol. Cell. Biol. 36, 965-978. 


\section{Acknowledgements}

I would like to thank Prof. Dr. Markus T. Bohnsack for giving me the opportunity to work on this project, and for his excellent supervision and support during the past four years.

To the additional members of my Thesis committee, Prof. Dr. Jörg Enderlein and Prof. Dr. Gerhard Braus, I am very grateful for their supervision and feedback on this project. I would also like to thank the examination board, Prof. Dr. Blanche Schwappach-Pignataro, Prof. Dr. Michael Thumm, Prof. Dr. Michael Meinecke and Prof. Dr. Ralf Ficner.

I want to heartfully thank Dr. Katherine Bohnsack for her guidance, encouragement and patience during my PhD. Thank you for fruitful scientific discussions, helpful suggestions and comments both during the $\mathrm{PhD}$ and the preparation of this thesis. I am very grateful to all the colleagues at the Department of Molecular Biology who make it such a nice working place.

To the members of the Bohnsack lab, former and recent, I am thankful for the nice working environment, their support and their help during the realisation of this thesis. I am deeply thankful to Philipp Hackert for his help and suggestions, especially when gigantic experiments got out of my small hands.

I want to specially thank soon-to-be Dr. Priyanka Choudhury, who accompanied me during this entire journey. Thank you for the much needed unfruitful, nonscientific discussions, and also for the brainstorming sessions and suggestions when things would not work out.

To my friends Marina, Priyanka, Javier, Nils, Jens, Nicolas, Julia and Jhon. Thank you all for your patience and support, for the many coffee sessions and neverending fun both inside and outside the institute. The most, I am thankful to Javi for helpful discussions, his honest opinions and his ENDLESS patience.

Thank you to Christian, Mariana (+ Hagen) and Marieke for welcoming me in Germany and always being a great source of support when I am homesick or frustrated. Without your open doors I wouldn't have made it so far.

Gracias totales to my beautiful group of berliners: Ale, Viole, Martin and occasionally Mati and Ceci. To have you all here, 11,000 km away from home, was 
the best surprise life could give me.

To Dr. Markus Kilisch, for his love and support every day, but specially during the preparation of this thesis. Te amo.

To the Kilisch family, Brigitte, Frank and Sarah, for welcoming me in the family with open arms, a cup of coffee and loads of love.

Finally, I am forever grateful to my parents, Claudia and Gastón, for their support and love during every step of my life that made me who I am. I am also immensely grateful to my brothers, Santi and Nachi, and to Vicky and Flori, for making my life that much louder and colourful regardless of the distance. I take you all with me wherever I go. 
